\title{
HOMOTOPICALLY STRATIFIED SETS
}

\author{
FRANK QUINN
}

The objective is to give a setting for the study of purely topological stratified phenomena, particularly group actions on manifolds. The "geometrically" stratified sets developed by R. Thom [21] and others [22] have given an effective context for smooth and PL phenomena (for example real semialgebraic sets are smoothly stratified, and polyhedra are PL stratified), but the topological version has been less successful. Among other problems, there are examples which are very nice locally but which do not admit global geometrically stratified structures. As a result stratified topological questions have had to be approached in a somewhat ad hoc fashion.

In a homotopically ${ }^{1}$ stratified set the strata are related by homotopy rather than geometric conditions. This makes them easier to construct and far more general, but makes it harder to see that they have any useful properties. Nonetheless they do; the principal results of this paper give extensions of isotopies, collars for boundaries, recognition theorems, an $h$-cobordism theorem, and obstructions to the existence of regular neighborhoods and geometric stratifications. The isotopy extension theorem, for example, implies that the skeleta are topologically homogeneous.

We give some background on stratifications, then discuss group actions, previous work, and the organization of the paper.

A geometrically stratified set is a filtered space $X^{0} \subset X^{1} \subset \cdots \subset X^{n}$ such that the strata are manifolds. "Skeleta" refers to the subsets $X^{i}$, "strata" the differences $X^{i}-X^{i-1}$.) The strata are also related to one another in a very precise way; if $k>i$ then a neighborhood of $X^{i}-X^{i-1}$ in $\left(X^{k}-X^{k-1}\right) \cup$ $\left(X^{i}-X^{i-1}\right)$ is a bundle of some kind, and there are compatibility conditions on these bundles at points where three or more strata come together. We get smooth, PL, or topological stratified sets depending on the types of strata and bundles used.

In a homotopically stratified set the strata are related by homotopy conditions. Roughly, neighborhoods of $X^{i}-X^{i-1}$ in $\left(X^{k}-X^{k-1}\right) \cup\left(X^{i}-X^{i-1}\right)$ have the local homotopy properties of mapping cylinders of fibrations. No compatibility conditions are required.

Received by the editors July 27, 1987 and, in revised form, January 7, 1988.

1980 Mathematics Subject Classification. Primary 57N80, 57S17, 57R80, 57Q10.

Partially supported by the National Science Foundation.

1 "Weakly" stratified sets in the preprint (May 1985) and [15]. 
Geometrically stratified sets (of any category) can often be manipulated by standard manifold techniques and induction on skeleta; after doing something to the $k$-skeleton, extend to a neighborhood in the $(k+1)$-skeleton using bundle theory, and then use manifold theory to manipulate the rest of the stratum. Stratified versions of surgery, transversality, and $h$-cobordism theorems are developed this way in [4]. Our treatment of homotopically stratified sets also proceeds by induction on strata, using the controlled topology of [13] instead of bundle theory to deal with neighborhoods of strata. The theory is very satisfactory except in one regard; strata of dimension less than 4 or 5 cannot be effectively manipulated. Consequently the structure theorems have restrictions on low dimensional strata.

Quotients of group actions on manifolds provide an important class of examples. Suppose a group $G$ acts on a manifold $M$. The orbit type filtration of $M$ is defined by: if $H \subset G$ and $V$ is a component of the set of points with isotropy subgroup $H$, then $V \subset M^{i}$ if $\operatorname{dim}(V) \leq i$.

If $G$ acts smoothly on $M$ then the quotient $M / G$ with the orbit type filtration is smoothly stratified. If $G$ acts piecewise linearly, then the quotient is PL stratified by orbit type if and only if the action satisfies a local product condition, satisfied for example if the action is locally linear. Therefore isotopy, $h$-cobordism, surgery theorems, etc. for smooth or PL actions follow from the corresponding theorems for smoothly or PL stratified sets.

If $G$ acts topologically then fixed sets need not be ANRs, let alone something nicely stratified. But even if we assume the action is locally linear the quotient is often not topologically stratified by orbit type. For example there are locally linear actions whose quotient cannot be geometrically stratified, because the singular set does not have an equivariant mapping cylinder neighborhood [13 II, 2.1.4]. Even when geometric structures exist they are not determined by the data, so "invariants" defined using them (e.g. Whitehead torsion) are often not well defined.

We define a group action to be "homotopically stratified" if the quotient with the orbit type filtration is a homotopically stratified set. Such actions are characterized by local conditions and include the locally linear ones. In fact they include nearly all actions with manifold fixed sets (1.6). The results of this paper specialize to give geometric properties for these actions. The flexibility of these actions also makes possible new directions of inquiry. For example $\mathrm{CW}$ actions on finite complexes are often concordant to ones whose only isotropy subgroups are $p$-groups and the whole group [14]. There can be no analog of this for locally linear actions because it is false for the linear ones. However it is reasonable to hope some version is valid for homotopically stratified actions.

We mention some previous work in contexts weaker than geometrically stratified sets. Siebenmann [17] has proved isotopy extension theorems for locally cone-like sets. Theorem 1.1 applies to more general spaces, but has a dimension 
restriction. Also [17] gives information about the space of isotopies, where 1.1 concerns single isotopies ( $\pi_{0}$ of the space).

Siebenmann's results are essentially local. Anderson and Hsiang have developed a global version with strata related by microbundles and obtained pseudoisotopy information [2] and obstructions to triangulation [3]. Again the results here apply more generally but give information about $\pi_{0}$ rather than the whole spaces of pseudoisotopies and triangulations.

There has been much work done on locally linear group actions by Madsen, Rothenberg, Dovermann, and many others. The theory presented here generalizes many of the basic results discovered in this context. For example Steinberger and West [19] describe an equivariant $h$-cobordism theorem for locally linear actions which "admit handle structures," and this has been extended to locally linear actions with "codimension $>2$ gaps" by Steinberger [18]. Theorem 1.8 applies to much more general actions, as well as stratified sets which do not arise from group actions.

$\S 1$ contains statements of the geometric results. In $\S 2$ the basic properties of homotopy links and tameness are developed. General homotopically stratified sets are defined in $\S 3$, and their basic structure described using the material of $\S 2$. The properties of ANR homotopically stratified sets, and layered sets, are developed in $\S 4$. This includes stratified versions of "finiteness" and simple homotopy theory, and connections with the controlled topology of [13]. $\S 5$ concerns manifold homotopically stratified sets, and provides proofs of the geometric theorems.

\section{Statements of Results}

In this section the geometric results are stated. Homotopy links are briefly described so that the geometric statements will make sense, but details are deferred to $\S \S 2$ and 3.

The "homotopy link" (holink $(X, Y)$; see 2.1$)$ is a topological analog of the boundary of a regular neighborhood in a PL pair. It is defined for any topological pair, and there are maps $(X-Y) \leftarrow \operatorname{holink}(X, Y) \rightarrow Y$, which are natural with respect to maps of pairs which preserve complements. There is a natural map from the homotopy pushout of these maps to $(X, Y)$, which is the identity on $Y$. (The homotopy pushout is the double mapping cylinder.) In the PL case if $N$ is a regular neighborhood of $Y$ then the holink diagram is homotopy equivalent to $(X-\operatorname{int}(N)) \leftarrow \partial N \rightarrow Y$. The homotopy pushout of the PL diagram is actually isomorphic to $X$. In general there is a "tameness" condition under which the pushout of the holink diagram is homotopy equivalent to $(X, Y)$ (by homotopies preserving complements; see 2.1).

A filtered space $X$ is homotopically stratified if $X^{i}-X^{i-1}$ is tame in $\left(X^{k}-X^{k-1}\right) \cup\left(X^{i}-X^{i-1}\right)$ when $k>i$, and the homotopy link of this pair is a fibration over $X^{i}-X^{i-1}$ (see 3.1). These fibrations are the homotopy analogs of the bundles relating strata in a geometrically stratified set. We remark that "compatibility conditions" involving three or more strata follow from this, so 
need not be assumed. For example it follows (see 3.2) that $X^{i}$ is tame in $X^{k}$, and the homotopy link is a stratified system of fibrations over $X^{i}$ in the sense of [13 II].

As above if $X$ is a filtered space the subsets $X^{i}$ are called the "skeleta" and the differences $X^{i}-X^{i-1}$ the "strata." A map $X \rightarrow Y$ preserves strata if every component of a stratum of $X$ has image in a stratum of $Y$. The image stratum is not required to have the same index, because the indexing is usually artificial. For instance an isovariant map of $G$-spaces induces a stratum-preserving map of quotients, but strata are taken to ones with the same isotropy subgroup rather than the same dimension.

We can now define the main objects of interest. A manifold homotopically stratified set is a locally compact homotopically stratified metric space $X$ whose strata are manifolds, which satisfy a boundary condition. Define the boundary $\partial X$ to be the union of the boundaries of the strata, $U_{i} \partial\left(X^{i}-X^{i-1}\right)$. Then $\partial X$ is required to be a stratum-preserving neighborhood deformation retract in $X$. Equivalently, $X \cup_{\partial X} \partial X \times[0,1)$ is homotopically stratified. In the official definition (5.1) the boundary condition is given in an equivalent "local" form which is more complicated but easier to verify.

The geometric results are stated next, beginning with ones which do not involve obstructions. $X, Y$, etc. are understood to be manifold homotopically stratified sets, with strata indexed by dimension.

1.1. Theorem. Suppose $Y \subset X$ is a closed union of components of strata which contains 4-dimensional strata. Then an isotopy of $Y$ which preserves strata and fixes the boundary extends to an isotopy of $X$ which preserves strata and fixes the boundary and the complement of a neighborhood of $Y$.

An "isotopy of $Y$ " is an isotopy which starts with the identity of $Y$. Isotopies which do not fix the boundary can be extended by "absorbing the boundary into the filtration" (5.1), or by using two applications of 1.1 and the next result.

1.2. Theorem. Suppose $\partial X^{4}$ has a stratum-preserving collar in $X^{4}$. Then $\partial X$ has a stratum-preserving collar in $X$.

We give a simple but useful application of the extension theorem. The interior of a connected manifold is "isotopically homogeneous" in the sense that any point can be isotoped to any other. The results above can be used to extend such isotopies to homotopically stratified sets.

1.3. Corollary. Suppose $x$ and $y$ are points in the same component of the interior (or of the boundary) of a stratum of $X$, and adjacent strata (or boundaries) have dimension at least 5 . Then there is a stratum-preserving isotopy of $X$ which moves $x$ to $y$. 
For example if $X$ is the quotient of a group action, and the action can be shown to be locally linear at a few strategically selected points, then it is locally linear everywhere (eg. [13 II, 2.1.4]).

We recall that isotopy homogeneity characterizes the PL intrinsic skeleta of a polyhedron [1]. In [15] ${ }^{2}$ the corollary is used to develop a topological version of intrinsic skeleta; it is shown that any manifold homotopically stratified set is a "subdivision" of a canonical minimal one, and the strata of this minimal structure can be effectively recognized from the given data.

Next we give criteria for group actions to be homotopically stratified; the most important is Corollary 1.6.

1.4. Theorem. Suppose $G$ is a discrete group which acts on a filtered metric space $X$, so that isotropy subgroups are locally constant on strata and $N(H) / H$ acts properly discontinuously on the set of points with isotropy subgroup $H$. Then $X$ is a manifold homotopically stratified set if and only if $X / G$ is, and isotropy subgroups of adjacent strata have finite index.

If $G$ is a finite group most of the hypotheses are automatic, and the statement is simply: filter $X$ by orbit type, then $X$ is a manifold homotopically stratified set if and only if $X / G$ is. The more general statement applies for example to actions resembling the action of a crystallographic group on euclidean space (see the example after 1.9). The theorem itself is a special case of a result about "multiply branched covers" (3.6).

This result means we can determine if the homotopically stratified theory applies to a group action (via its quotient) by inspecting the orbit type filtration of $X$. We do not have to produce slices, local product structures, etc. The next result often applies to the filtration of $X$.

1.5. Proposition. Suppose $M$ is a manifold with a finite collection $\left\{M_{s}\right\}$ of closed submanifolds such that the intersection of any two is again in the collection. Filter $M$ by $M^{i}=\bigcup\left\{M_{s} ; \operatorname{dim} M_{s}<i\right\}$. Then with this filtration $M$ is a manifold homotopically stratified set provided either

(1) if $M_{s} \subset M_{t}$ then it is 1-LC embedded, or

(2) the collection is locally flat in $M$.

Case (1) is the most useful because it is a homotopy condition; a subspace is 1-LC embedded if small loops in the complement extend to small maps of 2-disks in the complement. For manifolds this is equivalent to locally flat and not codimension 2 [13 I], so the point of case (2) is that codimension 2 subspaces are allowed. A collection is locally flat if each point has a neighborhood $U$ with a homeomorphism to $\mathbf{R}^{n}$ such that each $U \cap M_{t}$ is taken to a linear subspace. Note that there are no complicated transversality conditions like those needed in the geometrically stratified analogs.

2 There is an error in [15]; Theorem 2, p. 239, should be weakened to say there exists a filtered space $L_{X}$ with the stated property. Generally $L_{X}$ is not given by the construction preceeding the statement, although in the special situation where the theorem is used on p. 247 it does apply. 
We combine 1.4 and 1.5 in the case of finite groups.

1.6. Corollary. Suppose $G$ is a finite group acting on a manifold $M$, so that for all subgroups $G \supset H \supset J, M^{H}$ is a manifold locally flat and not codimension 2 in $M^{J}$. Then $M / G$ with the orbit type filtration is a manifold homotopically stratified set.

So (for example) by applying 1.3 in the quotient we see that components of strata are equivariantly isotopically homogeneous in $M$.

The remaining results involve obstructions. To explain the setting for these, suppose $p: E \rightarrow X$ is a map. Let $\mathscr{S}$ denote one of the spectrum-valued functors of spaces whose homotopy groups are $\pi_{1} \mathscr{S}(F)=\mathrm{Wh}\left(\pi_{1} F\right), \pi_{i} \mathscr{S}(F)=$ $\widetilde{K}_{i}\left(\mathbf{Z} \pi_{1} F\right)$ for $i \leq 0$. In [13 II] the "finite structure spectrum" constructed from pseudoisotopies is used. There is also a controlled version of algebraic $K$-theory (mentioned in [16]) whose associated "Whitehead spectrum" which would serve equally well. These functors differ in their higher homotopy groups, but this does not effect the homology groups used here.

A "spectral cosheaf" $\mathscr{S}(p) \rightarrow X$ is formed roughly by applying the functor $\mathscr{S}$ to $p$ fiberwise. Homology groups $H_{i}(X ; \mathscr{S}(p))$ with spectral cosheaf coefficients are defined in [13 II, $\S 8]$. If $X$ is locally compact, "locally finite" homology $H_{i}^{l f}$ is defined (and is often the reduced homology of the 1-point compactification). These groups can sometimes be computed using a spectral sequence which relates them to ordinary homology [13 II, $\S \S 1,8]$.

The first result concerns mapping cylinder neighborhoods in a single stratum. It is a direct consequence of [13 II, Theorem 1.1], and tameness properties (2.13, and the first lemma in 5.5).

1.7. Theorem. Suppose $X$ is a manifold homotopically stratified set and $i>5$. Then there is an invariant $q_{0}\left(X^{i}, X^{i-1}\right) \in H_{0}^{l f}\left(X^{i-1} ; \mathscr{S}\left(p_{i}\right)\right)$ which vanishes if and only if $X^{i-1}$ has a mapping cylinder neighborhood in $X^{i}$.

The map in the coefficient spectrum is $p_{i}$ : $\operatorname{holink}\left(X^{i}, X^{i-1}\right) \rightarrow X^{i-1}$. When $X$ is the quotient of a group action this is the obstruction to the existence of an equivariant mapping cylinder neighborhoods, see [13 II, §2.1]. These invariants are also, in the case of quotients of locally linear actions with "codimension $>2$ gaps," closely related to the "controlled equivariant finiteness" obstructions of Steinberger and West [20].

The next theorem is a basic statement of the $h$-cobordism theorem. The general setting for "stratified simple homotopy" is described in 1.10, after a discussion of the obstruction groups, and applications to group actions. A more elaborate $h$-cobordism theorem is given in $\S 5$.

An $h$-cobordism in this context is $\left(X ; Y_{0}, Y_{1}\right)$, where $X$ is a compact manifold homotopically stratified set, $\partial X=Y_{0} \cup Y_{1}$, and if $A$ is a stratum of $X$ then the inclusions $A \supset Y_{i} \cap A$ are homotopy equivalences. If $A$ is a stratum 
component $\delta A$ denotes the "frontier" (the complement of $A$ in its closure in $X$ ), and is a union of components of lower dimensional strata.

1.8. Theorem. Suppose $Y_{0}$ is a compact manifold homotopically stratified set, and let $\left\{A_{i}\right\}$ denote the components of the strata of $Y_{0}$. If $\left(X ; Y_{0}, Y_{1}\right)$ is a stratified h-cobordism then a "stratified Whitehead torsion"

$$
\tau(f) \in \sum_{i} H_{1}\left(\operatorname{cone} \delta A_{i}, \delta A_{i} ; \mathscr{S}\left(q_{i}\right)\right)
$$

is defined. If there is a stratum-preserving product structure on the 5-dimensional skeleton of $X$ then there is a stratum-preserving product structure $X \simeq Y_{i} \times I$ if and only if $\tau\left(X, Y_{0}\right)=0$.

The maps $q_{i}: E_{i} \rightarrow$ cone $\delta A_{i}$ in the coefficient spectrum $\mathscr{S}\left(q_{i}\right)$ are obtained by taking mapping cylinders of the horizontal maps, and identifying cone $\delta A$ with the mapping cylinder of $\delta A \rightarrow \mathrm{pt}$. :

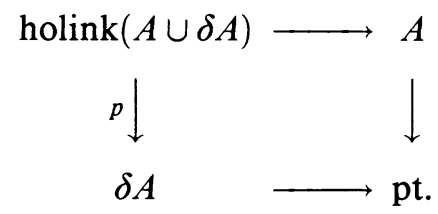

To explain the significance of the obstruction groups we consider the long exact sequence of the pair (cone $\delta A, \delta A$ ). The absolute groups of the cone are the groups of a point, which are the homotopy groups of the coefficient spectrum. Making this substitution, the exact sequence is:

$$
\begin{aligned}
\cdots \rightarrow H_{1}(\delta A ; \mathscr{S}(p)) \rightarrow \mathrm{Wh}\left(\pi_{1} A\right) & \rightarrow H_{1}(\operatorname{cone} \delta A, \delta A ; \mathscr{S}(q)) \\
& \rightarrow H_{0}(\delta A ; \mathscr{S}(p)) \rightarrow \widetilde{K}_{0}\left(\mathbf{Z} \pi_{1} A\right) \rightarrow \cdots
\end{aligned}
$$

Torsion invariants for geometrically stratified sets lie in the $\mathrm{Wh}\left(\pi_{1} A\right)$ term [4], and these can be thought of as $h$-cobordisms obtained by adding finitely many homotopically cancelling handles to the interior of the stratum. This geometric torsion is not "topologically invariant" since the existence of a product structure depends only on the image in the next group down in the sequence; the image of $H_{1}(\delta A ; \mathscr{S}(p))$ is lost. Roughly $H_{1}(\delta A ; \mathscr{S}(p))$ corresponds to infinite but controlled collections of handles near $\delta A$, so the image in $\mathrm{Wh}\left(\pi_{1} A\right)$ consists of elements which can be "pushed off the edge" of $A$ by a controlled infinite process.

On the other side of the $h$-cobordism group is the group $H_{0}(\delta A ; \mathscr{S}(p))$, in which lie the mapping cylinder obstructions of Theorem 1.7. These invariants are actually controlled finiteness obstructions, defined for finite dimensional ANRs as well as manifolds. The image of the torsion $\tau(f)$ is the difference of the end finiteness obstructions of $X$ and $Y$. For $h$-cobordisms what this means is roughly that if $X$ and $Y_{0}$ have the same mapping cylinder obstructions on a stratum, then there is a product structure near the frontier of the stratum. The 
nontrivial part of the $h$-cobordism is concentrated in the interior of the stratum, and the obstruction lies in the image of $\mathrm{Wh}\left(\pi_{1} A\right)$.

From this point of view, the reason ordinary Whitehead groups classify geometrically stratified $h$-cobordisms (even topological ones) is that mapping cylinder neighborhoods of strata are included as part of the structure.

We give a concrete application to group actions, where the obstructions can be arranged to vanish. This extends a result of Steinberger [18, Corollary 4] for locally linear actions. An "isovariant $h$-cobordism" is a $G$ manifold triple $\left(W, M, M^{\prime}\right)$ such that for all subgroups $G \supset H \supset J$ the inclusions $\left(M^{J}-M^{H}\right) \rightarrow\left(W^{J}-W^{H}\right) \leftarrow\left(M^{\prime J}-M^{\prime H}\right)$ are homotopy equivalences.

1.9. Corollary. Suppose $G$ is a finite group and $\left(W, M, M^{\prime}\right)$ a compact isovariant h-cobordism, so that for all subgroups $G \supset H \supset J, W^{H}$ is a manifold locally flat and codimension at least 3 in $W^{J}$. Suppose also that $M$ and each $M^{J}$ is 1-connected and nonempty. Then an equivariant product structure on fixed sets of dimension less than 5 extends to an equivariant product structure on $W$.

According to 1.6 the quotient $X=M / G$ of this action is a manifold homotopically stratified $h$-cobordism, so 1.8 applies. (We have not required product structures on 5-dimensional strata to be given since finite groups are "good"; see the remark below). The connectivity assumptions imply that the obstruction groups of 1.8 vanish, so product structures exist.

The reason the obstruction groups are trivial is as follows: Let $A$ be a stratum component, the quotient of a component in $W$ with isotropy subgroup $G_{W}$. Let $J$ denote $N G_{W} / G_{W}$, then under the codimension and connectivity restrictions $\pi_{1} A=J$. There is (see [13 II, 2.1]) a spectral sequence $H_{i}\left(\delta A ; \mathrm{Wh}_{j}\left(J_{x}\right)\right) \Rightarrow H_{i+j}\left(X^{n-1} ; \mathscr{S}(p)\right)$, where $\mathrm{Wh}_{j}$ denotes $\mathrm{Wh}$ if $j=1$ and $\tilde{K}_{j}$ if $j<1 . J_{x}$ is the system of isotropy subgroups over $\delta A$. Since the fixed sets are connected and nonempty, the $H_{0}\left(-; \mathrm{Wh}_{j}\right)$ term is just $\mathrm{Wh}_{j}(J)$. Because of this, the exact sequence for (cone $\delta A, \delta A$ ) (written out above) shows that the obstruction group goes injectively to the part of $H_{0}(\delta A ; \mathscr{S}(p))$ which comes from terms in the spectral sequence with $i>0$. The $H_{1}\left(-; \mathrm{Wh}_{1}\right)$ term vanishes by the simple-connectivity assumption on fixed sets. The others are trivial by Carter's vanishing theorem for lower $K$ groups [5].

We describe a much more sophisticated example. Suppose $G \subset L$ is a uniform, discrete, cocompact subgroup of a Lie group with finitely many components. Let $K \subset L$ be a maximal compact subgroup. The quotient $K \backslash L$ is a contractible manifold on which $G$ acts smoothly. If $G$ is torsion free then it acts freely on $K \backslash L$, and $K \backslash L / G$ is a manifold. If $G$ is not torsion free then the torsion subgroups occur as isotropy groups, and $K \backslash L / G$ is a smooth stratified set.

If $L$ is virtually solvable, so $G$ is poly-(finite or cyclic), and $G$ is torsion free then Farrell and Hsiang [9] have shown that $h$-cobordisms of $K \backslash L / G$ are products because the Whitehead group of $G$ is trivial. Now suppose $G$ does 
have torsion, and nontrivial fixed sets, but that if one fixed set is contained in another it has codimension at least 3 . Then the stratum with isotropy subgroup exactly $H$ has quotient with fundamental group $N(H) / H$, where $N$ denotes the normalizer in $G$. The obstruction group for equivariant smooth (or PL) $h$-cobordisms of this action is the sum $\sum \mathrm{Wh}(N H / H)$, where $H$ varies over conjugacy classes of finite subgroups of $G$. These are usually nonzero, containing for example Whitehead groups of finite subgroups. Using 1.8 we see that the homotopically stratified obstructions lie in $\sum \mathrm{Nil}_{1}\left((K \backslash L)^{H}, N H / H ; \mathbf{Z}\right)$, the generalized Nil groups of [16]. These are not always trivial, but there are much better vanishing results for them than for the Whitehead groups.

We return to the discussion of simple homotopy. In the unstratified case torsions are defined for homotopy equivalences of compact ANRs. Further, the torsion of a homotopy equivalence vanishes if and only if the map is homotopic to a composition of cell-like maps and their homotopy inverses [7], [6]. Recall that a map is cell-like if it is proper and each point inverse is contractible inside any neighborhood of it.

The objects in the stratified version are "layered sets," locally compact and finite dimensional homotopically stratified sets with ANR strata and reversetame skeleta. These are defined and discussed in 4.5 ; here we mention only that manifold homotopically stratified sets are layered, and the obstruction used in 1.8 is a special case of the next theorem.

1.10. Theorem. Let $\left\{A_{i}\right\}$ denote the components of strata of a compact layered set $Y$.

(1) A stratum-preserving map $f: X \rightarrow Y$ which is a homotopy equivalence on strata has a "Whitehead torsion" $\tau(f) \in \sum_{i} H_{1}\left(\operatorname{cone} \delta A_{i}, \delta A_{i} ; \mathscr{S}\left(q_{i}\right)\right)$.

(2) Given $\alpha$ in the obstruction group there is a compact layered set $X$ and a stratum-preserving homotopy equivalence $f: X \rightarrow Y$ with $\tau(f)=\alpha$.

(3) If $f: X \rightarrow Y$ and $g: Z \rightarrow X$ are stratum-preserving homotopy equivalences, then $\tau(f g)=\tau(f)+f_{*} \tau(g)$.

(4) If $f: X \rightarrow Y$ is a stratum-preserving homotopy equivalence of compact layered sets, then $\tau(f)=0$ if and only if there are $Z$ and stratumpreserving cell-like maps $g: Z \rightarrow X, h: Z \rightarrow Y$, and a stratified homotopy from $f g$ to $h$.

In (3) $f_{*}$ denotes the homomorphism, induced by $f$, from the $H_{1}$ groups of $X$ to those of $Y$.

Putting the parts of 1.10 together gives a "classification" result: Say that two maps $X_{i} \rightarrow Y, i=1,2$, are "CL equivalent" if there is $Z$ and stratumpreserving cell-like maps $Z \rightarrow X_{i}$ so that

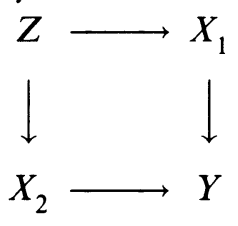


is stratified homotopy commutative. Then the theorem implies that this is indeed an equivalence relation, and that $\tau$ induces a bijection from the set of equivalence classes to the sum of homology groups.

The PL unstratified version of this "classification" was developed by Cohen [7] as a geometric approach to the Whitehead group. PL versions are much more elementary, so we mention that every layered set is the cell-like image of a PL one, and that if $X$ and $Y$ are both PL in (3) above, then $Z, f$, and $g$ can be chosen to be PL. Note that "PL" in this context means that the strata are polyhedra and does not imply that the space itself is even locally triangulable.

The unstratified ANR version is due to Chapman [6] and applies also to infinite dimensional ANRs. The restriction here to finite dimensions-a result of delicate dimension-dependent arguments in [13] - seems not to affect the applications.

There is also a manifold version, following essentially from $1.8 ; h$-cobordisms with a given compact manifold homotopically stratified set on one end are classified up to homeomorphism by the torsion of the inclusion. See 5.6.

The next characterizes layered sets, up to homotopy.

1.11. Theorem. Suppose $Y$ is a homotopically stratified set. $Y$ is stratified homotopy equivalent to a compact layered set if and only if the strata, and fibers of homotopy links of strata, are dominated by finite complexes, and for each stratum component $A$ the Wall finiteness obstruction $\sigma(A)$ is taken to 0 by

$$
\widetilde{K}_{0}\left(\mathbf{Z} \pi_{1} A\right) \rightarrow H_{0}(\text { cone } \delta A, \delta A ; \mathscr{S}(q)) .
$$

For unstratified objects the analog is the finiteness criterion of Wall [23] for spaces dominated by finite complexes. That result requires the vanishing of an invariant in $\widetilde{K}_{0}$, so by analogy one expects a stratified obstruction in the homology group $H_{0}$. The fact that it can be identified as the image of ordinary obstructions is a consequence of the "rigidity" of finiteness obstructions.

1.12. Geometric stratifications. When does a homotopically stratified set have a geometric stratified structure? If we use the geometric structures with topological strata and topological block bundles in the incidence data, the obstructions are the same as the obstructions to triangulation described in [13 II, 2.2.7], with the exception of the Kirby-Siebenmann obstruction for the strata. We mention these briefly, without a formal statement.

Suppose there is a structure given on $X^{i}$. There are three levels of obstructions encountered in extending it to $X^{i+1}$. First is the mapping cylinder obstruction of 1.7. If this vanishes there are obstructions to splitting the resulting map into pieces over mapping cylinders in the lower strata. If these vanish, the resulting pieces are approximate fibrations over lower strata. Last there are obstructions to these approximate fibrations being block bundles.

1.13. Low dimensions. The controlled boundary and $h$-cobordism theorems used in this development have been extended to dimension 4, provided the local 
fundamental groups in the coefficient maps are "good." This is done in [13 III] for "good" = trivial. It is extended in [11] to a class of groups containing poly(finite or cyclic) groups, and in particular finite groups. Consequently 1.2 gives collars in 4-strata provided local fundamental groups of $p$ : holink $\left(X^{4}, X^{3}\right) \rightarrow$ $X^{3}$ are "good." With similar "goodness" hypotheses 1.7 gives mapping cylinders in 5-strata, and product structures need only be assumed on 4-dimensional strata in 1.8. In particular this is the case in quotients of finite group actions, and this improvement is already incorporated in Corollary 1.9.

At present there are no results on controlled pseudoisotopy in dimension 4, so the isotopy results $1.1,1.3$ are not known to extend.

Finally we speculate that a 3-dimensional homotopically stratified set is triangulable. This would imply that many of the results hold for all strata except possibly 4-dimensional ones with bad fundamental groups.

\section{TAMENESS AND HOMOTOPY LINKS}

Proposition 2.2 and Corollary 2.3 show how homotopy links are used to construct, for tame subsets, the strict maps and homotopies which are central to the theory. Good information about links and tameness is therefore vital, and most of the section is devoted to developing this. The corollary to 2.4 gives relations between tameness and homotopy links in products, triples, and subsets. Proposition 2.6 concerns group actions, 2.8 treats unions. The most significant result for the structure of homotopically stratified sets is 2.9 , which is used to describe how unions of strata fit together. These results give tameness conclusions but with some tameness hypotheses. Initial input is provided by 2.10, which gives a homological characterization of tameness, and 2.12 which shows 1-LC subsets in ANRs are tame. Finally 2.13 relates the "tameness" used here to "reverse tameness," the notion used in controlled topology.

2.1. Definitions. Suppose $f: X \rightarrow Y$ is a map. The mapping cylinder will be denoted by cyl $(f)$, and we mean precisely the set $X \times(0,1] \cup Y \times\{0\}$ with the minimal topology such that the inclusion $X \times(0,1] \subset \operatorname{cyl}(f)$ and projection $\operatorname{cyl}(f) \rightarrow Y \times[0,1]$ are continuous. $Y$ is put at the 0 end of the mapping cylinder so that the $[0,1]$ coordinate is the "distance" from $Y$, as in polar coordinates. This topology is sometimes different from the quotient topology. A particular fact about it that we will use is that if $Z$ is a metric space and $G: X \times(0,1] \rightarrow Z, h: Y \rightarrow Z$ are continuous, then $G \cup h$ defines a continuous map $\operatorname{cyl}(f) \rightarrow Z$ if the restrictions $F_{t}: X \times\{t\} \rightarrow Z$ converge uniformly to $h f$ as $t$ goes to 0 .

A map $f:(A, B) \rightarrow(X, Y)$ is said to be a strict map of pairs if $f(B) \subset$ $Y$ and $f(A-B) \subset(X-Y)$. The space of strict maps (with the compactopen topology) is denoted $\operatorname{map}_{s}(A, B ; X, Y)$. Similarly strict maps of triples $(A, B, C) \rightarrow(X, Y, Z)$ preserve the indicated subsets and complements. 
The homotopy link ${ }^{3}$ is $\operatorname{holink}(X, Y)=\operatorname{map}_{s}([0,1],\{0\} ; X, Y)$. A point in holink $(X, Y)$ is therefore a path in $X$ which begins in $Y$ but immediately leaves it. There is a homotopy commutative diagram

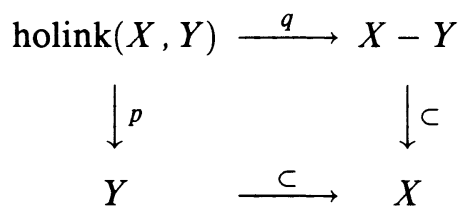

The maps $p$ and $q$ are given by evaluation at 0 and 1 respectively. The homotopy between the compositions is given by the evaluation map holink $(X, Y) \times$ $I \rightarrow X$. This diagram and homotopy are natural with respect to strict maps of pairs.

Let $Z$ denote the homotopy pushout of $p$ and $q$. (The homotopy pushout is the double mapping cylinder $\operatorname{cyl}(p) \cup_{\text {holink }} \operatorname{cyl}(q)$.) The homotopy in the diagram defines a strict map $(Z, Y) \rightarrow(X, Y)$. This map is universal with respect to strict maps of homotopy pushouts into $(X, Y)$. Since a pair is a homotopy pushout if and only if the subspace has a mapping cylinder neighborhood, this means the holink homotopy pushout is in an appropriate sense universal with respect to strict maps of mapping cylinders to $(X, Y)$.

The homotopy link is a topological analog of the frontier of a regular neighborhood in a PL pair. Suppose $(X, Y)$ is PL, and denote a regular neighborhood by $N$, with frontier $\partial N$. Then $N$ is the mapping cylinder of a map $\partial N \rightarrow Y$, and so $X$ is isomorphic to the homotopy pushout of $Y \leftarrow \partial N \rightarrow(X-$ int $N)$.

A subspace $Y \subset X$ is tame if there is a neighborhood $N$ and a strict map $(N \times I, Y \times I \cup N \times\{0\}) \rightarrow(X, Y)$ which is the identity on $Y$ and the inclusion on $N \times\{1\}$. Such a map is called a nearly strict deformation retraction of a neighborhood into $Y$. It is "nearly" strict in the sense that points in the complement of $Y$ do not get pushed into $Y$ until the last moment, when it is absolutely necessary. The homotopy can be used to define a strict homotopy inverse for the natural map of the holink pushout to the pair, and in fact $Y$ is tame if and only if the natural map (pushout, $Y) \rightarrow(X, Y)$ is a strict homotopy equivalence (see 2.4).

Example. Let $X$ be the 1-point union of countably many arcs, with lengths going to $0 ; X=\vee_{i=1}^{\infty}\{i\} \times\left[0, \frac{1}{i}\right]$, and let $Y=0$. Then $Y$ is tame in $X$.

We will see that tame subsets have many of the properties enjoyed by subsets with mapping cylinder neighborhoods. This $X$ is strictly rel $Y$ homotopy equivalent to the mapping cylinder of the map from a countable discrete set to a point ( holink $(X, Y)$ has countably many contractible components). However this $Y$ does not have a mapping cylinder neighborhood, and fails to have an important property of mapping cylinders; the reverse tameness of 2.13.

\footnotetext{
${ }^{3}$ This construction seems to have been first used by Fadell $[8, \S 4]$ to homotopically locate the normal bundle of a submanifold. It also appears in [13 II, §7.8].
} 
2.2. Proposition. Suppose $(A, B),(X, Y)$, and $f_{0}: B \rightarrow Y$ are given, and $B \subset$ $A$ is tame. Then strict homotopy classes of maps $(A, B) \rightarrow(X, Y)$ extending $f_{0}$ correspond to homotopy classes of morphisms of diagrams

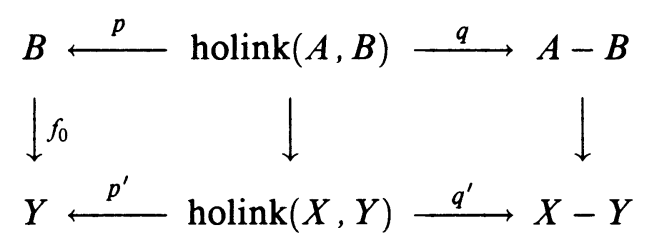

in which the left square commutes and a homotopy is given which makes the right square homotopy commute.

Proof. The naturality of the holink diagram associates commutative diagrams to strict maps. Conversely a "morphism" of diagrams induces a map of homotopy pushouts $(C, B) \rightarrow(Z, Y)$ which is strict because the left square commutes. There is the natural strict map $(Z, Y) \rightarrow(X, Y)$, and since $B$ is tame there is a strict homotopy inverse $(A, B) \rightarrow(C, B)$ (see the corollary to 2.4). Composing these with the induced map gives a strict map $(A, B) \rightarrow(X, Y)$. It is easily seen that these two constructions are inverses on the level of homotopy classes.

We think of the projection holink $(X, Y) \rightarrow Y$ as a sort of "normal bundle" for $Y$, and use some bundle terminology. We say a commutative diagram

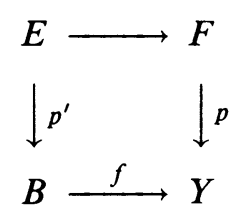

is a "fiber map" from $E$ to $F$, over $f$. So for example a strict map induces a fiber map of holinks. Fiber homotopy is defined similarly, and again strict homotopy induces fiber homotopy of holinks. The next result is a very useful converse to this last observation.

2.3. Corollary. Suppose $B$ is tame in $A$, and a strict map

$$
F_{0} \cup f:(A \times\{0\} \cup B \times I, B \times I) \rightarrow(X, Y)
$$

is given. Then it extends to a strict homotopy if and only if the fiber map of homotopy links over $f_{0}$ induced by $F_{0}$ extends to a fiber homotopy of homotopy links over $f$.

This follows from 1.2 and the observation that $\operatorname{holink}(A \times I, B \times I) \simeq$ holink $(A, B) \times I$.

We refine some of this slightly. Suppose $\delta: Y \rightarrow(0, \infty)$ is given, and define the controlled homotopy link, $\operatorname{holink}^{\delta}(X, Y)$, to be the set of $j$ in holink $(X, Y)$ with image within $\delta(j(0))$ of $j(0)$. 
2.4. Lemma. Suppose $X$ is metric, and $Y \subset X$ is closed.

(1) The inclusion $\operatorname{holink}^{\delta}(X, Y) \subset \operatorname{holink}(X, Y)$ is a fiber homotopy equivalence over $Y$.

(2) If $Y$ is tame and $\varepsilon: X \rightarrow(0, \infty)$ is given, then there is $\delta: Y \rightarrow(0, \infty)$ such that the natural map from the homotopy pushout of $Y \leftarrow$ $\operatorname{holink}^{\delta}(X, Y) \rightarrow X-Y$ to $X$ is a strict $\varepsilon$ homotopy equivalence rel $Y$. Conversely if the map is a strict homotopy equivalence rel $Y$ then $Y$ is tame.

An $\varepsilon$ homotopy equivalence is one such that the image of the homotopies in $X$ have radius less than $\varepsilon$. A homotopy equivalence "rel $Y$ " is one in which the maps and homotopies are all the identity on $Y$.

Proof. For (1) we observe that given $r$ : holink $(X, Y) \rightarrow(0,1]$ we can define $M_{r}: \operatorname{holink}(X, Y) \rightarrow \operatorname{holink}(X, Y)$ by composing each map $j \in \operatorname{holink}(X, Y)$ with multiplication by $r(j) . M_{t r+(1-t)}$ for $t \in[0,1]$ defines a fiber homotopy from the identity to $M_{r}$. If $r$ is small enough the image of $M_{r}$ lies inside $\operatorname{holink}^{\delta}(X, Y)$, and gives a homotopy inverse for the inclusion. We show that a small enough $r$ exists. For each $f \in \operatorname{holink}(X, Y)$ there is $r_{f}>0$ so that the restriction of $f$ to $\left[0, r_{f}\right]$ lies within $\delta(f(0))$ of $f(0)$. By continuity there is a neighborhood $U_{f}$ of $f$ so that for $g$ in the neighborhood $g\left[0, r_{f}\right]$ lies within $\delta(g(0))$ of $g(0)$. Cover holink $(X, Y)$ by such neighborhoods, say $U_{f_{\alpha}}$, and choose a partition of unity $\theta_{\alpha}$ subordinate to this cover. Then $r=\sum_{\alpha} \theta_{\alpha} r_{f_{\alpha}}$ has the desired property.

For the proof of (2) suppose $r: N \times I \rightarrow X$ is a nearly strict retraction of a neighborhood to $Y$. Choose $\delta: Y \rightarrow(0, \infty)$ so that: if $y \in Y$ and $d(x, y)<\delta(y)$ then the arc $r(x, I)$ is defined and has radius $<\varepsilon$. Define $s: X \rightarrow[0, \infty)$ by $s(x)=\inf \{d(x, y) / \delta(y), y \in Y\}$. Note $s^{-1}(0)=Y$ and $s(x)<1$ iff there is $y$ such that $d(x, y)<\delta(y)$. The homotopy pushout can be written as $(X-Y) \times\{1\} \cup \operatorname{holink}^{\delta}(X, Y) \times I \cup Y \times\{0\}$, with identifications induced by evaluating paths in the holink at 0 and 1 . The natural map to $X$ is given by projection of the first and third pieces, and evaluation on the second. To go back, take $x$ to $(x, 1)$ if $s(x) \geq 1,(x, 0)$ if $s(x)=0$, and to $(r(x, I), s(x))$ if $s(x) \leq 1$. Homotopies of the two compositions to the identities are easily constructed from $r$, and by choice of $\delta$ have radius $<\varepsilon$.

The converse of (2) follows from the observations that strict homotopy equivalence rel $Y$ preserves tameness of $Y$, and that since $Y$ has a mapping cylinder neighborhood in the pushout it is tame there.

Corollary. In the following we assume the spaces are metric and the subsets are closed.

(1) $Y \subset X$ is tame if and only if $Y \times Z \subset X \times Z$ is tame. Further, the inclusion holink $(X, Y) \times Z \subset$ holink $(X \times Z, Y \times Z)$, is a fiber homotopy equivalence over $Y \times Z$. 
(2) If $X \supset Y \supset Z$ and $Y \subset X$ is tame, then $(Y-Z) \subset(X-Z)$ is tame. Further, holink $(X-Z, Y-Z)=p^{-1}(Y-Z)$, where $p$ is the projection $p: \operatorname{holink}(X, Y) \rightarrow Y$.

(3) If $X \supset N \supset Y$ and $N$ contains a neighborhood of $Y$, then $Y \subset$ $X$ is tame if and only if $Y \subset N$ is tame. Further, the inclusion $\operatorname{holink}(N, Y) \subset \operatorname{holink}(X, Y)$ is a fiber homotopy equivalence over $Y$.

Statements (1) and (2) and the tameness part of (3) are elementary. The holink part of (3) follows from 2.4(1).

Tameness is a local property. Define locally tame to mean that each point in $Y$ has a neighborhood $V$ in $X$ so that $V$ nearly strictly deformation retracts rel $V \cap Y$ into $Y$.

2.5. Lemma. Suppose $X$ is a metric space. Then $X \supset Y$ is locally tame at each point if and only if it is tame.

Proof. Cover $Y$ with sets $V_{i} \subset X$ with almost strict deformations $r_{i}: V_{i} \times I \rightarrow$ $X$ into $Y$. Extend these to $X \times\{1\} \cup Y \times I$ by the identity. Let $N$ denote the number (possibly infinite) of sets $V_{i}$, and define a map $R_{N}$ on a subset of $X \times I^{N}$ to $X$ by composition: Let $R_{1}(x, t)=r_{1}(x, t), R_{2}\left(x, t_{1}, t_{2}\right)=$ $r_{2}\left(R_{1}\left(x, t_{1}\right), t_{2}\right)$, and inductively $R_{i}\left(x, t_{1}, \ldots, t_{i}\right)=r_{i}\left(R_{i-1}\left(x, t_{1}, \ldots, t_{i-1}\right), t_{i}\right)$. Then define $R_{N}=\lim _{i \rightarrow N} R_{i}$. We will only use this when all but finitely many of the $t_{i}$ are 1 (and thus $r_{i}$ is the identity), so nothing sophisticated about limits is needed.

Next, if functions $a_{i}: X \rightarrow[0,1]$ are given we can define a function $R(x, t)$ on a subset of $X \times I$ by restricting $R_{N}$ to the line in $I^{N}$ from $\left(a_{i}(x)\right)$ to (1). Explicitly, $R(x, t)=R_{N}\left(x, t+(1-t) a_{1}(x), \ldots, t+(1-t) a_{i}(x), \ldots\right)$. Now choose functions $a_{i}$ so that $a_{i}=1$ on the complement of $V_{i}$, the product $\prod_{i} a_{i}=0$ in a neighborhood of $Y$, and so that any point has a neighborhood in which all but finitely many of the $a_{i}$ are identically $i$. Such functions are constructed in the same way as partitions of unity, using the fact that $X$ is metric and $\left\{V_{i}\right\}$ cover a neighborhood of $Y$. This map $R$ satisfies $R(x, 1)=$ $x$, and where it is defined in the neighborhood where the product of the $a_{i}$ vanishes, it gives a nearly strict deformation into $Y$.

To complete the proof we show that $R$ is defined on a neighborhood of $Y$. Choose a point $y \in Y$, and let $U$ be a neighborhood so that there are finitely many indices $i(1), \ldots, i(k)$ so that for $i$ not in this set $a_{i}$ is 1 on $U$, and for $i$ in the set $r_{i}$ is defined on $U \times I$. Define $R^{i}$ to be the restriction of $R_{i}$ to the appropriate arc; $R^{i}(x, t)=r_{i}\left(R^{i-1}(x, t), t+(1-t) a_{i}(x)\right)$, when defined. Suppose $U_{j} \subset U$ is a neighborhood of $y$ so that $R^{i(j)}$ is defined on $U_{j} \times I$. Since $R^{i(j)}(y, t)=y$ for all $t$, and $U$ is a neighborhood of $y$, there is a neighborhood $U_{j+1} \subset U_{j}$ of $y$ so that $R^{i(j)}\left(U_{j} \times I\right) \subset R$. For indices $i(j)<i<i(j+1) \quad a_{i}=1$ on $U$, so $R^{i(j)}=R^{i(j+1)-1}$. Since $r_{i}(j+1)$ is 
defined on $U, R^{i(j+1)}(x, t)=r_{i(j+1)}\left(R^{i(j)}(x, t), t+(1-t) a_{i(j+1)}(x)\right)$ is defined for $x \in U_{j+1}$. Since $R=R^{i}(k)$ on $U_{k}, R$ is defined on $U_{k}$ as required.

The next result is used in the study of group actions and "branched covers"; see 3.6.

2.6. Proposition. Suppose $X, X^{*}$ are metric spaces and $X$ is locally finite dimensional. If $\left(X^{*}, Y^{*}\right) \rightarrow(X, Y)$ is strict, open, strongly light, has the unique lifting property for strict arcs, and the restriction $X^{*}-Y^{*} \rightarrow X-Y$ is a covering space, then $Y^{*} \subset X^{*}$ is tame if and only if $Y \subset X$ is tame. Further, holink $\left(X^{*}, Y^{*}\right)$ is the pullback of

$$
X^{*}-Y^{*} \rightarrow X-Y \leftarrow \operatorname{holink}(X, Y) .
$$

Explanations. Denote the map by $q$. The arc lifting property is that if $j:(I, 0)$ $\rightarrow(X, Y)$ is strict and $x \in X^{*}$ is a lift of $j(1)$, then there is a unique lift of $j$ beginning at $x$. Strongly light means each point $z \in X^{*}$ has arbitrarily small neighborhoods $U$ containing no other points in the preimage of $q(z)$ and such that $U$ is separated from $q^{-1} q(U)-U$. ("Light" traditionally means point inverses are separated from each other. Here we are more specific about the separating sets.)

These hypotheses are not independent. For example if $X-Y$ is locally 1-connected then the path lifting hypothesis implies $q$ is a covering space over $X-Y$. Also a covering space is strongly light, so this hypothesis is unnecessary except near $Y$.

Proof. The first step is to establish the continuity of the lifting property. Suppose $j: E \times(I, 0) \rightarrow(X, Y)$ and a lift of $E \times\{1\}$ are given. The lifting property implies there is a lift $j^{*}$ of $j$ extending this, which is continuous on arcs $\{e\} \times I$. Since $q$ is a covering space in the complement of $Y, j^{*}$ is continuous on $E \times(0,1]$. To show it is continuous at $(e, 0)$ choose a neighborhood of $j^{*}(e, 0)$. There is a smaller one, say $V$, which satisfies the strongly light property. Since $q$ is open $q(V)$ is a neighborhood of $j(e, 0)$, and therefore contains the image of $j(W \times J)$, where $W$ is a neighborhood of $e$ and $J$ an interval containing 0 . Since $j^{*}$ is continuous on arcs and on $E \times(0,1]$, there are a smaller neighborhood $W^{\prime}$ and interval $[0, \delta]$ so that $j^{*}$ takes $W^{\prime} \times\{\delta\} \cup\{e\} \times[0, \delta]$ into $V$. But now $j^{*}$ takes all the arcs $\{w\} \times[0, \delta]$ into $q^{-1} q(V)$, and one end into $V$, which is separated from $q^{-1} q(V)$. Therefore $j^{*}\left(W^{\prime} \times[0, \delta]\right) \subset V$. This implies $j^{*}$ is continuous.

The continuous lifting property is equivalent to the holink statement; the lifting property gives a lift of the pullback into the holink, and the uniqueness of lifts implies it is a homeomorphism. Similarly suppose $Y \subset X$ is tame, and $r: N \times I \rightarrow X$ is a nearly strict retraction of a neighborhood. Let $N^{*}=q^{-1}(N)$, then we can lift $r$ on $N-Y$ to a strict map $\left(N^{*}-Y^{*}\right) \times(I, 0) \rightarrow\left(X^{*}, Y^{*}\right)$. To see this extends continuously to the identity on $Y^{*}$ we need: arcs which start in a sufficiently small neighborhood of $y \in Y^{*}$, and have small diameter when 
mapped into $X$, have small diameter in $X^{*}$. The reason for this is almost the same as that for continuity.

Finally we show that $Y^{*} \subset X^{*}$ tame implies $Y \subset X$ tame, by showing that $Y^{*}$ is locally tame at any particular point. Since $X$ is locally finite dimensional we may restrict to a neighborhood, and assume finite dimensionality.

Suppose $X$ has dimension $n$, and we want to show local tameness at $y \in Y$. Let $R: V \times I \rightarrow X^{*}$ be a nearly strict deformation of a neighborhood of $y^{*}$ into $Y^{*}$, where $q\left(y^{*}\right)=y$. Choose neighborhoods $N_{n+1} \supset N_{n} \supset \ldots \supset N_{0}$ of $y^{*}$ in $X^{*}$ which satisfy the strongly light property and $R\left(N_{i} \times I\right) \subset N_{i+1}$. Choose open sets $U_{0}, \ldots, U_{n} \subset q\left(N_{0}\right)-Y$ so that $Y \cup\left\{U_{i}\right\}$ is a neighborhood of $y$, and each $U_{i}$ lifts into $N_{0}$. These are obtained as follows: Since $X^{*}-Y^{*} \rightarrow X-Y$ is a covering space, each point in $q\left(N_{0}\right)-Y$ has a neighborhood which lifts into $N_{0}$. Choose a locally finite covering $\left\{V_{\alpha}\right\}$ of $q\left(N_{0}\right)-Y$ by such sets. Since $X$ has dimension $n$ there are open sets $U_{0}, \ldots, U_{n}$ such that each $U_{i}$ is a union of disjoint open sets each contained in some $V_{\alpha}$. Lifts defined independently on these disjoint open sets give a continuous lift of $U_{i}$.

We now construct, by induction, nearly strict deformations $s^{i}$ of $\bigcup_{j \leq i}\left\{U_{i}\right\}$ into $Y$, inside $q\left(N_{i+1}\right)$. Assume $s_{i}$ is defined, then there is a lift

$$
S_{i}:\left(\bigcup_{j \leq i}\left\{U_{i}\right\} \cap U_{i+1}\right) \times I \rightarrow X^{*}
$$

of the restriction of $s_{i}$, extending the lift of $U_{i+1}$ into $N_{0}$. By the strongly light property of $N_{i+1}, S_{i}$ has image in $N_{i+1}$. Now "splice" together $R$ and $S_{i}$. The composition $R\left(S_{i}(-,-),-\right):\left(\bigcup_{j \leq i}\left\{U_{i}\right\} \cap U_{i+1}\right) \times I \times I \rightarrow X^{*}$ has image in $N_{i+2}$. Define $S_{i+1}:\left(\bigcup_{j \leq i}\left\{U_{i}\right\} \cap U_{i+1}\right) \times I \rightarrow X^{*}$ by restricting this; choose $a, b: X \rightarrow$ $[0,1]$ and for each $u$ restrict to the straight line in $I \times I$ from $(0,0)$ to $(a(u), b(u))$. If the product $a b$ is 0 then this gives an almost strict deformation into $Y^{*}$. If $a$ is 1 off $\bigcup_{j<i}\left\{U_{i}\right\}$ then $S_{i+1}$ extends continuously to $U_{i+1} \times I$ by $R$ off $\left.\bigcup_{j \leq i}\left\{U_{i}\right\} \cap U_{i+1}\right) \times \bar{I}$. If $b$ is 1 off $U_{i+1}$ then $q S_{i+1}$ extends continuously to $\bigcup_{j \leq i}\left\{\bar{U}_{i}\right\} \times I$ by si off $\left.\bigcup_{j \leq i}\left\{U_{i}\right\} \cap U_{i+1}\right) \times I$. Therefore if all three conditions are satisfied we can define $s_{i+1}$ to be $s_{i}$ on $\left(\bigcup_{j \leq i}\left\{U_{i}\right\}-U_{i+1}\right) \times I, q S_{i+1}$ on $\left(\bigcup_{j \leq i}\left\{U_{i}\right\} \cap U_{i+1}\right) \times I$, and $q R$ on $\left(U_{i+1}-\bigcup_{j \leq i}\left\{U_{i}\right\}\right) \times I$. We note it is impossible for all three conditions to be satisfied on the complement of $\bigcup_{j \leq i+1}\left\{U_{i}\right\}$, so in fact we get $s_{i+1}$ defined on a slightly smaller open subset of $\bigcup_{j \leq i+1}\left\{U_{i}\right\}$. It is easily arranged that there are such subsets so that their union with $Y$ is still a neighborhood of $y$. Therefore at the end of the construction we get a deformation $s_{n}$ of a neighborhood of $y$ into $Y$, and conclude that $Y \subset X$ is locally tame.

The next proposition (2.8) is a little less precise than the above in that it describes a holink only "approximately." To prepare for this we develop some notation. 
2.7. Approximate fiber maps. Suppose $p: F \rightarrow Y$ is a map. An approximate lift of a map $g: E \rightarrow Y$, to $F$, is a map $h: E \times(0,1] \rightarrow F$ so that $p h \cup$ $g: E \times[0,1] \rightarrow Y$ is continuous. If $q: E \rightarrow X$ is a map then an approximate fiber map covering $f: X \rightarrow Y$ is an approximate lift of $f q$ to $F$. Similarly an approximate fiber homotopy,covering a homotopy $f$, is a map $h: E \times I \times$ $(0,1] \rightarrow F$ such that $p h \cup f: E \times I \times[0,1] \rightarrow Y$ is continuous. There are approximate analogs of all the usual definitions; for example an approximate fiber homotopy equivalence is an approximate fiber map, an approximate fiber map going back, and approximate fiber homotopies of the two compositions to the identities.

Approximate fiber maps (or homotopies) define strict maps (or strict homotopies) of mapping cylinders. (This is a consequence of the topology we are using on the mapping cylinder.) So for example Proposition 2.2 could be generalized by allowing the center vertical map to be an approximate fiber map over $f_{0}$. Curiously, this is not really a generalization because for homotopy links the two notions are equivalent:

Lemma. An approximate lift of a map into $Y$ to $\operatorname{holink}(X, Y)$ is approximately fiber homotopic to a lift, relative to subsets where it is already a lift.

We say an approximate lift $B \times(0,1] \rightarrow \operatorname{holink}(X, Y)$ is "already a lift" if it is independent of the $(0,1]$ coordinate, i.e. factors through a map $B \rightarrow$ holink $(X, Y)$. This map is necessarily an exact lift of the original map to $Y$.

Proof. Let $h$ denote an approximate lift of $f: B \rightarrow Y$. The adjoint of $h$ is a strict map $h^{*}:(B \times(0,1] \times I, B \times(0,1] \times\{0\}) \rightarrow(X, Y)$. Since $h$ is an approximate lift the union $h^{*} \cup f: B \times(0,1] \times I \cup B \times\{0\} \times\{0\} \rightarrow X$ is continuous. Composition of this with the diagonal $B \times I \rightarrow B \times((0,1] \times I \cup\{0\} \times\{0\})$ gives an adjoint to an exact lift of $f$. Composition with the retraction in the first coordinate of $(0,1] \times I \cup\{0\} \times\{0\}$ to the diagonal gives an approximate homotopy of the original approximate lift to the exact one. Finally if $h$ is a product on some subset $C \subset B$ then this construction does not change $h$ on $C$.

This implies that holinks which are approximately fiber homotopy equivalent are in fact genuinely fiber homotopy equivalent. Similarly a homotopy link which is an approximate fibration, is in fact a fibration. The weaker notion of approximate fibration is still useful, for example in 2.8 because the pushout used there is not a homotopy link.

Recall that if $Y$ is a metric space, $\varepsilon: F \rightarrow(0, \infty)$ is given, and $p, g$ are as in the definition of 2.7, then an " $\varepsilon$ lift" of $g$ to $F$ is a map $j: E \rightarrow F$ such that $d(p j(x), g(x))<\varepsilon(x)$. Approximate fibrations are usually defined (e.g. in [13 I, 3.3]) to be maps with the $\varepsilon$ homotopy lifting property for all $\varepsilon$. This is equivalent to the approximate homotopy lifting property defined above. An $\varepsilon$ lift can be obtained by restricting an approximate lift. Conversely approximate 
lifts can be constructed by choosing $\varepsilon$ lifts for a sequence of $\varepsilon$ converging to 0 , and then using a relative form of the lifting property to fill in between them.

2.8. Proposition. Suppose $X$ is a metric space, $X \supset Y, X_{1}, X_{2}$ are closed, and let $Y_{i}=X_{i} \cap Y$. Suppose $\left(X_{1} \cap X_{2}, Y_{1} \cap Y_{2}\right)$ is a strict neighborhood deformation retract in both $\left(X_{1}, Y_{1}\right)$ and $\left(X_{2}, Y_{2}\right)$. Then $Y \subset X$ is tame if and only if $Y_{i} \subset X_{i}$ are tame, and in this case $Y_{1} \cap Y_{2} \subset X_{1} \cap X_{2}$ is tame. Further, holink $\left(X_{1} \cup X_{2}, Y_{1} \cup Y_{2}\right)$ is approximately fiber homotopy equivalent over $Y$ to the homotopy pushout of

$$
\operatorname{holink}\left(X_{1}, Y_{1}\right) \leftarrow \operatorname{holink}\left(X_{1} \cap X_{2}, Y_{2} \cap Y_{1}\right) \rightarrow \operatorname{holink}\left(X_{2}, Y_{2}\right) .
$$

Proof. The tameness conclusion is obtained by simple combinations of the given homotopies, so we concentrate on the homotopy link. We show that the natural map from the pushout has an $\varepsilon$ homotopy inverse, for every $\varepsilon>0$.

Let $R:(X, Y) \times I \rightarrow(X, Y)$ be a strict homotopy which preserves $X_{i}$, is fixed on $\left(X_{1} \cap X_{2}\right)$, and satisfies $R_{0}=\mathrm{id}$, and $R_{1}(N) \subset\left(X_{1} \cap X_{2}\right)$ for some neighborhood $N$ of $\left(X_{1} \cap X_{2}\right)$. Further suppose that the restriction of $R$ to $Y$ has radius less than $\varepsilon$. The existence of such an $R$ is equivalent to the strict deformation hypothesis. Choose $\delta: Y \rightarrow(0,1]$ with the property: If $y \in Y_{1} \cap Y_{2}, z \in Y$ and $x \in X$ such that $d(y, z)<\delta(y)$ and $d(z, x)<\delta(z)$, then $x \in N$. Define a homotopy $R^{\delta}: \operatorname{holink}^{\delta}(X, Y) \times I \rightarrow \operatorname{holink}(X, Y)$ by composition with $R$. Then we claim $R_{1}^{\delta}$ factors through the homotopy pushout indicated in the statement.

Here the homotopy pushout can be written holink $\left(X_{1}, Y_{1}\right) \times\{0\} \cup$ $\operatorname{holink}\left(X_{1} \cap X_{2}, Y_{2} \cap Y_{1}\right) \times I \cup \operatorname{holink}\left(X_{2}, Y_{2}\right) \times\{1\}$.

Define $s: Y \rightarrow[0, \infty)$ by $s(z)=\min \left\{d(y, z) / \delta(y), y \in Y_{1} \cap Y_{2}\right\}$. Note if $s(z)<1$ then there is $y$ with $d(y, z)<\delta(y)$, so by definition of $\delta$ any $j$ in holink $^{\delta}(X, Y)$ with $j(0)=z$ has image inside $N$. Consequently if $s j(0)<1$, $R_{1}^{\delta}(j)$ is in $\operatorname{holink}\left(X_{1} \cap X_{2}, Y_{2} \cap Y_{1}\right)$. If $s j(0)>1, R_{1}^{\delta}(j)$ is in exactly one of holink $\left(X_{1}, Y_{1}\right)$ or holink $\left(X_{2}, Y_{2}\right)$. Define $S: \operatorname{holink}^{\delta}(X, Y) \times I \rightarrow$ (pushout) by $S(j)=\left(R_{1}^{\delta}(j), t\right)$, where $t=0$ if $s j(0) \geq 1$ and $j(0) \in Y_{1}, t=1$ if $s j(0) \geq 1$ and $j(0) \in Y_{2}$. If $s j(0) \leq 1, t=(1-s j(0)) / 2$ if $j(0) \in Y_{1}$, and $t=(1+s j(0)) / 2$ if $j(0) \in Y_{2}$. The composition of $S$ with the natural map (pushout) $\rightarrow \operatorname{holink}(X, Y)$ is $R_{1}^{\delta}$.

If we compose $S$ with a homotopy inverse for the inclusion $\operatorname{holink}^{\delta}(X, Y) \subset$ holink $(X, Y)$ we get a homotopy inverse for the natural map (pushout) $\rightarrow$ $\operatorname{holink}(X, Y)$. The homotopy $R^{\delta}$ constructed above gives a homotopy of the composition in holink $(X, Y)$ to the identity, and the other homotopy is obtained similarly. Finally we note that since $R$ was assumed to have radius less than $\varepsilon$ on $Y$, the projections of these homotopies into $Y$ have radius less than $\varepsilon$, as required.

The next proposition relates holinks in a triple $X \supset Y \supset Z$, and is a converse of sorts to part (2) of the corollary to 2.4. It is less precise than the other results 
in that it compares the holink over $Y$ to something over an approximation to $Y$.

The homotopy link of the triple is defined by $\operatorname{holink}(X, Y, Z)=$ $\operatorname{map}_{s}(I \times I, I \times\{0\},\{0,0\}: X, Y, Z)$. Restrictions to $\{1\} \times I, I \times\{0\}$, and $\{0\} \times I$ define maps to $\operatorname{holink}(X-Z, Y-Z)$, $\operatorname{holink}(Y, Z)$, and holink $((X-Y) \cup Z, Z)$ respectively. These give a commutative diagram

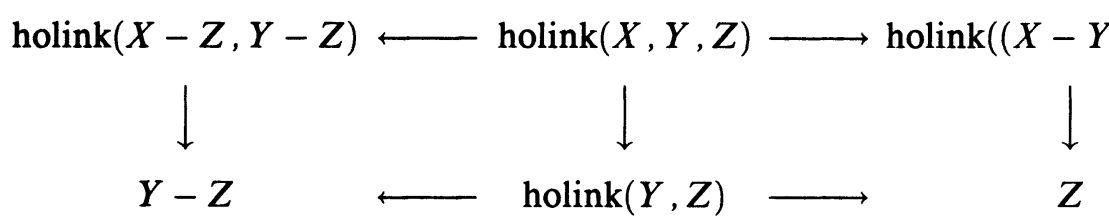

The homotopy pushout of the lower row is an approximation to $Y$, according to 2.2. The proposition asserts that the pushout of the upper is an approximation to holink $(X, Y)$. More precisely we use the controlled versions of the middle two terms, so that 2.4(2) applies to the lower pushout.

2.9. Proposition. Suppose $X \supset Y \supset Z$ are closed, $X$ is a metric space, $r: N \times$ $I \rightarrow Y$ is a nearly strict deformation retraction of a neighborhood of $Z$ in $Y$, and the restriction $r:(N-Z) \times(0,1] \rightarrow Y-Z$ is covered by a fiber map holink $(X-Z, Y-Z) \times(0,1] \rightarrow \operatorname{holink}(X-Z, Y-Z)$ which is the identity over 1. Then

(1) $Y \subset X$ is tame if and only if $Y-Z \subset X-Z$ and $Z \subset(X-Y) \cup Z$ are tame, and in this case $Z \subset X$ is tame. Further,

(2) given $\varepsilon: Y \rightarrow(0, \infty)$ there is $\delta: Y \rightarrow(0, \infty)$ such that the natural transformation from the map of homotopy pushouts of the rows of $\operatorname{holink}(X-Z, Y-Z) \longleftarrow \operatorname{holink}^{\delta}(X, Y, Z) \longrightarrow \operatorname{holink}((X-Y) \cup Z, Z)$

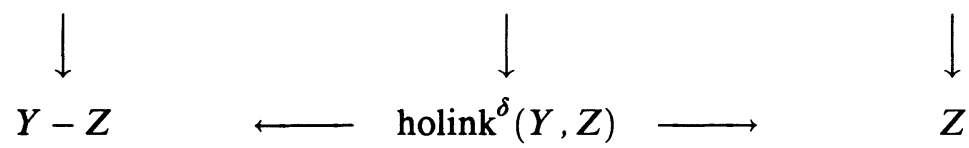

to holink $(X, Y) \rightarrow Y$ is an $\varepsilon$ homotopy equivalence. Moreover the left square is $\varepsilon$ homotopy equivalent to a pullback, and

(3) if $Y \subset X$ is tame then the natural map from the homotopy pushout of $\operatorname{holink}(Y, Z) \leftarrow \operatorname{holink}(X, Y, Z) \rightarrow \operatorname{holink}((X-Y) \cup Z, Z)$

to $\operatorname{holink}(X, Z)$, is a fiber homotopy equivalence over $Z$.

Proof. We begin by showing that if $Y-Z \subset X-Z$ is tame we may assume it has a pinched neighborhood in $X$ which is the mapping cylinder of holink $(X-Z, Y-Z) \rightarrow Y-Z$.

Proposition 2.4(2) applies to $Y-Z \subset X-Z$. Thus given $\beta: X \rightarrow[0, \infty)$ with $\beta^{-1}(0)=Z$ there is an $\alpha: Y \rightarrow[0, \infty)$ with $\alpha^{-1}(0)=Z$, so that the map from the pushout of $X-Y \leftarrow \operatorname{holink}^{\alpha}(X-Z, Y-Z) \rightarrow Y-Z$, to $X-Z$, is a $\beta$ homotopy equivalence strictly rel $Y-Z$. Let $P$ denote this pushout, 
with the following metric: The mapping cylinder is naturally homeomorphic to holink $^{\alpha}(X-Z, Y-Z) \times[0, \alpha]$, by which we mean the union of $\{f\} \times[0, \alpha f(0)]$ for $f \in$ holink, and on this we use the product metric. On $X-Y$ and $Y-Z$ use the given metrics. Then this extends to a metric on $P \cup Z$ by the given metrics on $X$ and $Y$, and by interpolating linearly on the arcs $\{f\} \times[0, f(0)]$. Denote this space by $P \hat{\cup} Z$, then the map $P \hat{\cup} Z \rightarrow X$ is a $\beta$ homotopy equivalence strictly relative to $Y$. Since holinks and tameness are preserved by such maps it is sufficient to consider these in $P \hat{\cup} Z$.

We now have a nice pinched neighborhood of $X-Z$. As a first application of this modification we see there is a strict homotopy $R:(X, Y) \times I \rightarrow(X, Y)$ with $R_{1}$ the identity and the restriction to a neighborhood of $Z$ in $Y$ the nearly strict retraction $r$ specified in the data. In the statement $r$ was only assumed to be defined on $N$, but it can be extended to the rest of $Y$ essentially to be the identity. Over $(0,1] r$ is covered by a fiber homotopy of holink ${ }^{\alpha}(X-Z, Y-Z)$ (using 2.4(2) to substitute the controlled holink in the hypothesis). The fiber homotopy gives a homotopy of the mapping cylinder in $P \hat{\cup} Z$ which is the identity on holink ${ }^{\alpha}(X-Z, Y-Z) \times\{1\}$ and $r$ on $Y$. This extends by the identity to give $R:(P \hat{\cup} Z) \times I \rightarrow P \hat{\cup} Z$ as required, and the strict equivalence gives such an $R$ on $X$.

Now we verify (1). If $Y \subset X$ is tame then $Y-Z \subset X-Z$ is tame by (2) of the corollary to 2.4 , so there is a map $R$ as above. Let $N$ be the neighborhood of $Z$ in $Y$ which $R$ deforms into $Z$, and let $W$ be a neighborhood of $Z$ in $X$ so that the nearly strict deformation of $X$ into $Y$ carries $W$ into $N$. The composition of $R$ and the deformation to $Y$ defines a map $W \times I \times I \rightarrow X$. The restriction to the diagonal is a nearly strict deformation of $W$ to $Z$, which preserves $(W-Y) \cup Z$, so $Z \subset X$ and $Z \subset(X-Y) \cup Z$ are both tame.

Conversely suppose $Y-Z \subset X-Z$ and $Z \subset(X-Y) \cup Z$ are tame, and conclude first that $Z \subset X$ is tame. There is a nearly strict deformation $S$ of $W \subset(X-Y) \cup Z$ to $Z$. There is also a deformation $R$ of a pinched neighborhood $V$ of $Y$ which is $r$ on $Y$, nearly strict with respect to $Z$, keeps the complement of $Y-Z$ in the complement, and pushes the intersection with a neighborhood of $Z$ into $Z$. To see this recall that we may assume (by using $P \hat{U} Z)$ that $Y$ has a pinched neighborhood which is the mapping cylinder of the holink. Then $R$ may be defined to be the mapping cylinder of the fiber map over $r$. The composition $S R$ is defined $(V-Y) \times I \times I \rightarrow X$. Suppose $a, b: X-Z \rightarrow[0,1]$ are given. Define $S^{*}: X \times I \rightarrow X$ by $R$ on $Y, S$ on $X-V$, and if $x$ is in $V-Y,\{x\} \times I$ is taken to $S R$ restricted to the straight line from $(x, 1,1)$ to $(x, a(x), b(x))$. This is continuous if $a$ is 1 on $X-V$ and $b$ is 1 on $Y$. It takes a neighborhood of $Z$ nearly strictly into $Z$ if the product $a b=0$. These three conditions can be satisfied on $X-Z$, so $S^{*}$ can be defined there. It extends continuously by the identity to $Z$.

Several observations about this $S^{*}$ will be useful later. First, it keeps $(X-Y) \cup Z$ in itself. This is because $R, S$ do. Second, on $Y$ it restricts to the retraction $r$ given in the data. 
To complete the proof of (1) we show that $Y \subset X$ is tame. Let $S^{*}: X \times$ $I \rightarrow X$ denote the nearly strict deformation constructed above, which pushes a neighborhood $W$ into $Z$, and is nearly strict with respect to the subspace $Y$. Let $T: X \times I \rightarrow X$ denote a nearly strict deformation which pushes a pinched neighborhood $V$ of $(Y, Z)$ into $Y$. Suppose $\delta: Y \rightarrow[0, \infty)$ is such that $\delta^{-1}(0)=Z$ and $V$ contains the ball of radius $3 \delta(y)$ about $y$. Both $T^{1}$ and $S^{1}$ are required to be the identity.

The composition defines a map $S^{*} T: X \times I \times I \rightarrow X ; S^{*} T(x, a, b)=$ $S^{*}(T(x, a), b)$. A new deformation will be defined by restricting this. Define $s(x)=\inf \{d(x, y) / \delta(y), y \in Y-Z\}$, and define

$$
a(x)=\max \{1, \min \{s(x)-2,0\}\},
$$

and

$$
b(x)=1-\max \{1, \min \{s(x)-1,0\}\} .
$$

Define a new homotopy $R:(X-Z) \times I \rightarrow X$ by mapping $\{x\} \times I$ to the straight line between $(a(x), b(x))$ and $(1,1)$, and composing with $S^{*} T$. Since $s$ is continuous on $X-Z, R$ is also. Since $S^{*}$ and $T$ both are the identity on $Z$, $R$ extends continuously by the identity on $Z$. On $Y-Z$ it is $T$, which is the identity, so $R$ is the identity on all of $Y$. Since $S^{*}$ and $T$ are nearly strict with respect to $Y$, so is $R$. Finally we observe that $R$ pushes a neighborhood of $Y$ into $Y$. On the pinched neighborhood of radius $2 \delta, a(x)=0$ so $T$ takes points into $Y$ and $S^{*}$ does not move them back out. Since $T$ fixes $Z$ there is a neighborhood $U$ of $Z$ which $T$ keeps inside $W$. On the complement of the $2 \delta$ pinched neighborhood in $U, b(x)=0$ so the $S^{*}$ part of $R$ takes points into $Z$. The union of the $2 \delta$ pinched neighborhood and $U$ is a neighborhood of $Y$, and is pushed into $Y$.

We now begin proof of (2). The equivalence of the bottom pushout to $X$ is given by 2.4 , and the upper part is very similar. We construct a map $\operatorname{holink}^{\delta}(X, Y) \rightarrow$ (pushout). Note that $p^{-1}(Y-Z)=\operatorname{holink}^{\delta}(X-Z, Y-Z)$ and $p^{-1}(Z)=\operatorname{holink}^{\delta}((X-Y) \cup Z, Z)$, where $p$ : $\operatorname{holink}^{\delta}(X, Y) \rightarrow Y$ is the projection. These map to the homotopy pushout by inclusion, so to fill in between these we need a map of a deleted neighborhood of $\operatorname{holink}^{\delta}((X-Y) \cup Z, Z)$ to the mapping cylinder part of the pushout, $\operatorname{holink}^{\delta}(X, Y, Z) \times I$. This is constructed from the fiber map of holinks covering the deformation $r$.

Extend $r$ (by the identity) to all of $Y ; r: Y \times I \rightarrow Y$. Let

$$
R: \operatorname{holink}^{\delta}(X-Z, Y-Z) \times(0,1] \rightarrow \operatorname{holink}^{\delta}(X-Z, Y-Z)
$$

denote a fiber map covering $r$. We have used 2.4(1) to substitute the controlled holink. For each $j \in \operatorname{holink}^{\delta}(X-Z, Y-Z)$ this gives a strict map

$$
R(j):((0,1] \times I,(0,1] \times\{0\}) \rightarrow(X-Z, Y-Z),
$$

which is $r(j(0),-)$ on $(0,1] \times\{0\}$. Extend this to $\{0,0\}$ using $r$, and compose with a standard map $I \times I \rightarrow(0,1] \times I \cup\{0,0\}$ which is the identity on $I \times\{0\}$. 
Denote the result by $\widetilde{R}(j): I \times I \rightarrow X$. If $j(0) \in N$ (the neighborhood $r$ pushes into $Z)$ then $\widetilde{R}(j)$ is a strict map $(I \times I, I \times\{0\},\{0,0\}) \rightarrow(X, Y, Z)$. In other words $\widetilde{R}(j)$ is a lift of $j$ into $\operatorname{holink}^{\delta}(X, Y, Z)$. This together with the function $s:(N-Z) \rightarrow I$ defined in the proof of 2.4 gives the required map into $\operatorname{holink}^{\delta}(X, Y, Z) \times I$. All together these define a map $\operatorname{holink}^{\delta}(X, Y) \rightarrow$ (pushout). It is easily verified, as in 2.4 , that for appropriately small $\delta$ this is an $\varepsilon$ homotopy inverse for the natural map.

Next we verify that the square

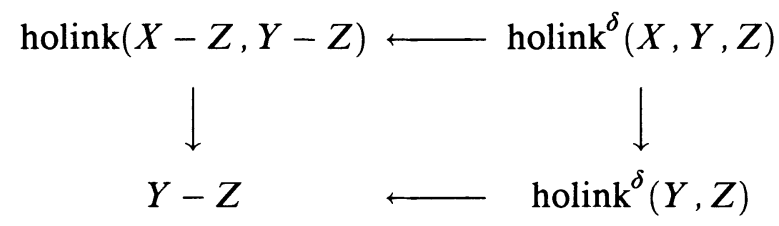

is $\varepsilon$ equivalent to a pullback. A point in the pullback is a pair $i, j$ with $i:(I, 0) \rightarrow(X-Z, Y-Z)$ strict, $j:(I, 0) \rightarrow(Y, Z)$ strict, and $i(0)=j(1)$. A map $I \times I \rightarrow X$ in the triple holink determines such a pair by restriction to $\{1\} \times I, I \times\{0\}$. The map $\widetilde{R}(i)$ constructed above gives a map from the pullback to the triple holink by taking $i, j$ to $i, r(i(0),-)$. To show that $\widetilde{R}$ is an $\varepsilon$ homotopy inverse for the restriction we need a canonical $\varepsilon$ homotopy from $j$ to $r(j(1),-)$. This is provided by $r(j(-),-)$, since $r(-, 1)$ is the identity.

Finally we prove (3). Since $Y \subset X$ is tame then there is a deformation $S^{*}$ as above, which pushes a neighborhood $V$ of $Z$ into $Z$. Also there is a nearly strict deformation into $Y$ of the pinched neighborhood of $Y$ of radius $\alpha$, where $\alpha: Y \rightarrow[0, \infty)$ has $\alpha^{-1}(0)=Z$. Let $R$ denote this deformation. We define a map $V-Z \rightarrow$ (pushout): If $x$ has radius at least $\alpha$ from $Y$ then $x$ is taken to the $\operatorname{arc} S^{*}(x,-) \in \operatorname{holink}((X-Y) \cup Z, Z)$. If $x$ is in $Y$ the same formula defines an $\operatorname{arc}$ in $\operatorname{holink}(Y, Z)$. If $x$ has radius $d$ from $Y$, with $0<$ $d<\alpha$, define $f x(s, t)=S^{*}(R(x, s), t)$. Then $\left(f_{x}, d / \alpha\right) \in \operatorname{holink}(X, Y, Z) \times$ $I$. These fit together to give the desired map to the pushout. This map of a neighborhood induces a map from $\operatorname{holink}(X, Z)$ to the pushout, which is easily seen to be a fiber homotopy inverse for the natural map.

The next objective is to develop a criterion which in useful circumstances implies tameness.

2.10. Homological tameness. Suppose $X$ is metric, $Y \subset X, M$ is a neighborhood of $Y$, and $\pi_{1}(M-Y) \rightarrow \pi$ is a homomorphism. Then $Y$ is homologically tame, with $\mathrm{Z} \pi$ coefficients, if there is a retraction $r: N \rightarrow Y$ of a neighborhood to $Y$, and for every neighborhood $U$ of $Y$ and $\varepsilon>0$ there is $V \subset U$ such that

$$
H_{i}\left(r^{-1}(K) \cap V, K ; \mathbf{Z} \pi\right) \rightarrow H_{i}\left(r^{-1}\left(K^{\varepsilon}\right) \cap U, K \varepsilon ; \mathbf{Z} \pi\right)
$$

is trivial, for every $i$ and $K \subset Y$. 
Here, as in [13], $K^{\varepsilon}$ denotes the set of points within $\varepsilon$ of $K$. The $\mathbf{Z} \pi$ homology is the ordinary homology of the branched cover corresponding to the kernel of the homomorphism to $\pi$ in the complement of $Y$. We do not require connectedness here. If $U, V$, etc. are not connected, $\pi_{1}$ is understood to be the groupoid obtained by disjoint union of fundamental groups of components, and similarly $\pi$ may be a groupoid.

A way to see the relevance of this condition is to suppose $Y$ is tame, so there is a nearly strict deformation of a neighborhood $V$ into $Y$, inside a neighborhood $U$. If $K \subset Y$ this restricts to give a nearly strict homotopy of $r^{-1}(K) \cap V$ into $Y$, rel $K$. If $V$ is small enough this takes place in $r^{-1}\left(K^{\varepsilon}\right) \cap U$, so the inclusion $\left(r^{-1}(K) \cap V, K\right) \rightarrow\left(r^{-1}\left(K^{\varepsilon}\right) \cap U, K^{\varepsilon}\right)$ is trivial in homology.

For $\mathbf{Z}$ coefficients this argument does not use strictness, so in fact a neighborhood deformation retract is $\mathbf{Z}$ homologically tame. If $\pi$ is nontrivial the strictness is used to lift the deformation into the covering space of $U-Y$ corresponding to the kernel of the homomorphism to $\pi$.

There is a corresponding notion of local homological tameness at a point $y \in Y$, namely there is a neighborhood $M$ of $y$ in $X$ so that the above condition holds for $K \subset M \cap Y$. We usually use the local version, since local fundamental groups are more often locally constant than globally constant.

2.11. Proposition. Suppose $X$ is metric, and $y \in Y \subset X$. If $Y$ is locally tame at $y$ then it is homologically locally tame at $y$, with any coefficients. Conversely if $X$ is finite dimensional, $Y$ and $X-Y$ are ANRs, and the complement of $Y$ has constant local fundamental group $\pi$ in a neighborhood of $y$, and $Y$ is homologically locally tame at $y$ with $\mathrm{Z} \pi$ coefficients, then $Y$ is locally tame at $y$.

The complement has constant local fundamental group $\pi$ near $y$ if there is a neighborhood $M$ in $X$ and $\pi_{1}(M-Y) \rightarrow \pi$, and a smaller neighborhood $N$ with the following property: Given $\varepsilon>0$ there is $\delta>0$ so that a loop in $N-Y$ of diameter less than $\delta$ has a nullhomotopy of diameter less than $\varepsilon$ in $M-Y$ if and only if it has trivial image in $\pi$. The condition "1-LC" for embeddings is equivalent to trivial local fundamental groups in this sense.

Proof. That tame implies homologically tame follows from the remarks given in the explanation of the definition.

The converse is proved using the controlled homotopy theory of [13 I, $\S 5]$. Suppose $Y$ is homologically tame over a neighborhood $N$ of $y$ in $Y$. The half-way point in the proof is to show that given a neighborhood of $Y, U$ and $\varepsilon>0$ there is $V \subset U$ such that for every $W \subset V$ there is $Z \subset W$ such that there is a homotopy of radius $<\varepsilon$ (measured in $Y$ ) of $(V-Y) \cap r^{-1}(N-\varepsilon)$, rel $Z-Y$, in $U-Y$, into $W-Y$.

To complete the proof from the half-way point, choose $\varepsilon_{i}>0$ so that $\varepsilon=$ $\sum \varepsilon_{i}$ exists, and $N^{-\varepsilon}$ is a neighborhood of $y$. Choose $V_{i}$ neighborhoods of $Y$ so that $\cap V_{i}=Y$, and there is a deformation of radius $<\varepsilon_{i}$ of $\left(V_{i}-Y\right) \cap$ $r^{-1}\left(N^{-\varepsilon_{i}}\right)$, rel $V_{i+2}$, in $V_{i-1}$, into $V_{i+1}$. Then define $H$ to be the limit of the 
concatination $h_{1} h_{2} \cdots h_{i} \cdots$. More precisely suppose $h_{i}(-, 1)$ is the identity and $h_{i}(-, 0)$ takes $V_{i}$ into $V_{i+1}$. Define $g_{j}=h_{j}\left(g_{j-1}(x), 0\right)$, and define $H(x, t)=h_{i}\left(g_{j-1}(x), i(i+1)(t-1 / i)\right)$, if $t$ is in $[1 / i, 1 / i+1]$. Then $H$ extends to the required nearly strict deformation of $(V-Y) \cap r^{-1}\left(N^{-\varepsilon}\right)$ into $Y$.

We describe how to get to the half-way point. To simplify notation we treat the case $N=Y$. Since $X$ is a finite dimensional ANR it can be arbitrarily closely approximated by $n$-complexes, for some fixed $n$. Therefore it is sufficient to show that for every $U$ and $\varepsilon>0$ there is $V \subset U$ so that for every $W \subset V$ there is $Z \subset W$ (all neighborhoods of $Y$ ) such that the inclusion $(V-Y, Z-Y) \rightarrow(U-Y, W-Y)$ is $(\varepsilon, n)$-connected over $Y$, in the sense of [13 I, 5.1]. According to the eventual Hurewitz theorem [13 I, 5.2] this is the case if we can find appropriate $V_{1} \supset \cdots \supset V_{k} \supset W_{k} \supset \cdots \supset W_{1}$ for sufficiently large $k$. The condition required of these is that $\left(V_{i+1}-Y, W_{i+1}-Y\right) \rightarrow\left(V_{i}-Y, W_{i}-Y\right)$ be $\delta$ homologically trivial with $\mathrm{Z} \pi$ coefficients, and $V_{i+1}-Y \rightarrow V_{i}-Y, W_{i+1}-Y \rightarrow$ $W_{i}-Y$ have "local image" in $\pi_{1}$ equal to $\pi$ (see [13 I, 5.2]). These conditions are easily achieved using the homological and local fundamental group hypotheses.

2.12. Corollary. Suppose $X$ is a locally finite dimensional metric $A N R$, and $Y \subset X$ is a closed ANR with trivial local fundamental group in the complement. Then $Y$ is tame in $X$.

Proof. Since $X$ and $Y$ are ANRs there is a neighborhood which deformation retracts to $Y$. This implies homological tameness with $Z$ coefficients, according to the comments after the definition, so 2.11 implies $Y$ is tame.

Note that if $Y$ and $X-Y$ are ANRs then $Y$ is a neighborhood deformation retract in $X$ if and only if $X$ is an ANR. If the local fundamental groups in the complement of $Y$ are constant, then 2.12 and 2.6 show that $Y$ is tame in $X$ if and only if the branched cover (branched over $Y$ ) is an ANR.

2.13. Reverse tameness. Suppose $X \supset Y$ are metric, and $r: N \rightarrow Y$ is a retraction of a neighborhood. $Y$ is said to be reverse tame in $X$ if for every $\varepsilon: Y \rightarrow(0, \infty)$ and neighborhood $U$ of $Y$ there are a neighborhood $V$ of $Y$ and a homotopy $h:(X-Y) \times I \rightarrow X-Y$ satisfying:

(1) $h$ is the identity on $(X-Y) \times\{0\} \cup(X-U) \times I$,

(2) $h$ takes $(U-Y) \times I$ into $U-Y$,

(3) $h((X-Y) \times\{1\}) \subset X-V$, and

(4) $r h$ has radius less than $\varepsilon$.

The homotopy in the definition of "tame" given in 2.1 pulls neighborhoods of $Y$ toward-and finally into- $Y$. The property here is called "reverse tame" because the opposite happens; the homotopy $h$ pulls $X-Y$ away from $Y$. 
If $X$ is locally compact then reverse tameness is the same as "tameness of the end of $X-Y$ over $Y$ " of definition 1.1 of [13 I]. It is needed in $\S 5$ to apply the results of [13] and is also used in $\S 4$ in the development of "simple homotopy."

2.14. Proposition. Suppose $X$ is locally compact, $Y \subset X$ is closed with locally constant fundamental group in the complement, and $X-Y$ is a manifold without boundary. Then $Y$ is tame in $X$ if and only if it is reverse tame.

Proof. Roughly, the two conditions are dual. With constant local fundamental group hypothesis there are homological characterizations of both notions of tame; 2.11 and [13 I, 1.7]. In terms of neighborhoods $U \supset V$ of $Y$ as above, the definition in 2.10 concerns $H_{i}(U, V)$ where $[13 \mathrm{I}, 1.6]$ uses $H_{i}(X-V, X-U)$. Homological tameness (either version) for $X-Y$ implies "tameness" for the corresponding cohomology groups. But one version of tameness for cohomology is Alexander dual to the other version of tameness in homology, so the two conditions are equivalent. Essentially this argument, with more detail, is given in the proof of Theorem 3.1.1 of [13 I].

There is another criterion for reverse tameness, in terms of the homotopy link. Basically it says that the homotopy link must be very large for reverse tameness to fail. A consequence (actually an ingredient in the proof) is that for locally compact spaces, reverse tameness of a subset $Y$ is preserved by strict rel $Y$ homotopy equivalence.

2.15. Proposition. Suppose $X$ is locally compact and metric and $Y \subset X$ is closed and tame. Then $Y$ is reverse tame if and only if $\operatorname{holink}(X, Y) \rightarrow Y$ is properly $\varepsilon$ dominated, for all $\varepsilon: Y \rightarrow(0, \infty)$.

"Properly $\varepsilon$ dominated" means there is $r: K \rightarrow$ holink, $j$ : holink $\rightarrow K$, and a homotopy $h: r j \sim 1$ so that $p h r$ is proper and $p h$ radius less than $\varepsilon$. This condition allows construction of a locally compact model for the mapping cylinder of the holink.

Proof. Suppose first that $Y$ is reverse tame in $X$ and $\varepsilon$ is given. Use the tameness to choose a closed neighborhood $N$ which nearly strictly $\varepsilon / 4$-deformation retracts to $Y$. This deformation gives a map of $N-Y$ into $\operatorname{holink}^{\varepsilon / 4}(X, Y)$. Using the local compactness we may assume the deformation is proper, and in particular the retraction is proper.

Next suppose $\delta$ is small enough that the $\delta$ neighborhood of $Y$ lies inside $N$. Then there is a factorization

$$
\operatorname{holink}^{\delta}(X, Y) \rightarrow(N-Y) \rightarrow \operatorname{holink}^{\varepsilon / 4}(X, Y)
$$

which is $\varepsilon / 2$ homotopic to the identity. This means $N-Y \varepsilon / 2$-dominates holink $(X, Y)$. Now use reverse tameness to find a $\delta$ homotopy of $N-Y$ into $N-U$, for some open neighborhood $U$ of $Y$. This gives a $\delta$ domination 
of $N-Y$, so if $\delta$ is small enough gives an $\varepsilon$ domination of $\operatorname{holink}(X, Y)$. Finally note that since $U$ is open the domination is also proper.

For the converse we define the mapping telescope of a sequence of dominations of $p: E \rightarrow Y$. Suppose we are given spaces $K_{i}$, maps $r_{i}: K_{i} \rightarrow E$ and $j_{i}: E \rightarrow K_{i}$, and homotopies $h_{i}: r_{i} j_{i} \sim 1$. Define $Z$ to be the union of the mapping cylinders of the compositions $K_{i} \rightarrow E \rightarrow K_{i+1}$, and $Y$. Explicitly, take $\bigcup_{i} K_{i} \times\left[\frac{1}{i+1}, \frac{1}{i}\right]$, identify points in $K_{i} \times\left\{\frac{1}{i+1}\right\}$ with images in $K_{i+1} \times\left\{\frac{1}{i+1}\right\}$. Then take the union with $Y \times\{0\}$. Give this the topology so that the inclusions and projection to $Y \times[0,1]$ (defined below) are continuous.

Define a map $Z \rightarrow \operatorname{cyl}(p)$ by: On $K_{i} \times\left\{\frac{1}{i+1}\right\} \rightarrow E \times\left\{\frac{1}{i+1}\right\}$ use the projection $r_{i}$. On the mapping cylinder of $j_{i+1} r_{i}$ to $E \times\left[\frac{1}{i}, \frac{1}{i+1}\right]$, fill in with the homotopy $-h_{i+1} r_{i}: r_{i} \sim r_{i+1} j_{i+1} r_{i}$. (Here " $-h$ " denotes the homotopy obtained by reversing the $I$ coordinate in $h$.) Finally define the map to be the identity $Y \times\{0\} \rightarrow Y \times\{0\}$. The "projection to $Y \times[0,1]$ " used to define the topology on the telescope is the composition of this map and the projection $\operatorname{cyl}(p) \rightarrow Y \times[0,1]$. The definition of the topology on the mapping cylinder implies that $Z \rightarrow \operatorname{cyl}(p)$ is continuous.

2.16. Lemma. Suppose $Y$ is locally compact metric, $p: E \rightarrow Y$ is given, $\varepsilon_{i}>0$ converge to 0 , and $\left(K_{i}, r_{i}, j_{i}, h_{i}\right)$ are proper $\varepsilon_{i}$ dominations of $E$ over $Y$. Denote the mapping telescope by $Z$. Then the map $Z \rightarrow \operatorname{cyl}(p)$ is a strict rel $Y$ homotopy equivalence, $Z$ is locally compact, and $Y$ is reverse tame in $Z$.

Proof of the lemma. A homotopy inverse $\operatorname{cyl}(p) \rightarrow Z$ is defined by $j_{i}$ on $E \times\left\{\frac{1}{i}\right\} \rightarrow K_{i} \times\left\{\frac{1}{i}\right\}$, and filling in with the homotopies $j_{i+1} h_{i}: j_{i+1} r_{i} j_{i} \sim j_{i+1}$. The convergence hypothesis on $\varepsilon_{i}$ implies that this is continuous. We describe a rel $Y$ homotopy of the composition $Z \rightarrow \operatorname{cyl}(p) \rightarrow Z$ to the identity of $Z$ (the homotopy of the other composition is similar and easier, so will not be described).

The map $Z \rightarrow Z$ is obtained from homotopies making the diagram

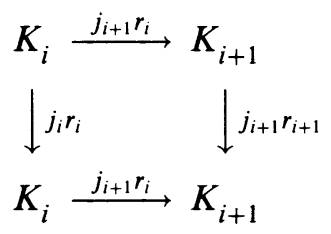

commute. This homotopy is the concatination of $j_{i+1} h_{i} r_{i}$, which takes $j_{i+1} r_{i} j_{i} r_{i}$ to $j_{i+1} r_{i}$, and $j_{i+1}\left(-h_{i+1}\right) r_{i}$ which takes $j_{i+1} r_{i}$ to $j_{i+1} r_{i+1} j_{i+1} r_{i}$, by inverting the homotopy parameter. The map of mapping cylinders can therefore be described as a concatination; $\left(j_{2} h_{1} r_{1}\right) \cdots\left(j_{i+1} h_{i} r_{i}\right)\left(-j_{i+1} h_{i+1} r_{i}\right)\left(j_{i+2} h_{i+1} r_{i+1}\right)$

There is a homotopy from the identity of $Z$ to the map which moves everything down one place in the union of mapping cylinders. On the diagram level this map corresponds to composing with $j_{i+1} r_{i}$. We compose this with the map $Z \rightarrow Z$. Specifically, in the range of the concatination we push the 
second homotopy in each pair down one level, and in the domain we push down into the domain of the first homotopy. This produces a homotopy to $\cdots\left(j_{i+1} h_{i} r_{i}\left[j_{i} r_{i-1}\right]\right)\left(-\left[j_{i+2} r_{i+1}\right] j_{i+1} h_{i+1} r_{i}\right)\left(j_{i+2} h_{i+1} r_{i+1}\left[j_{i+1} r_{i}\right]\right) \cdots$. Now using the homotopies $r_{*} j_{*} \sim 1$ this is homotopic to the concatination $\cdots\left(-j_{i+2} h_{i+1} r_{i}\right)$ $\cdot\left(j_{i+2} h_{i+1} r_{i}\right) \cdots$. This is homotopic, by cancellation of inverses of homotopies, to the map of mapping cylinders induced by the commutative diagrams

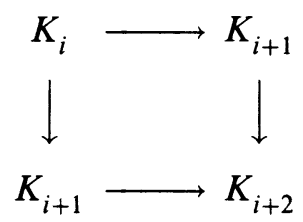

This map, however, is obtained by pushing down in the chain of mapping cylinders, so as above is homotopic to the identity.

This completes the proof that $Z$ is equivalent to $\operatorname{cyl}(p)$. This general sort of argument comes from [10], and is used in [13 II, 6.3].

Next we show $Y$ is reverse tame in $Z$. First define a retraction $R_{n}$ of $Z-Y$ to the first $n$ stages (the mapping cylinders of $K_{1} \rightarrow \cdots \rightarrow K_{n}$ ) by: On $K_{i}$ with $i>n$ use $j_{n} r_{i}: K_{i} \rightarrow K_{n}$. Then map the mapping cylinder of $K_{i} \rightarrow K_{i+1}$ to $K_{n}$ by the homotopy $-j_{n} h_{i+1} r_{i}: j_{n} r_{i} \sim j_{n} r_{i+1}\left(j_{i+1} r_{i}\right)$.

There is a homotopy $R_{n} \sim R_{n+1}$ : Push the $K_{i}$ mapped by $R_{n}$ into $K_{n}$ down into $K_{n+1}$. This corresponds to composing the homotopies used with $j_{n+1} r_{n}$, so we get $-j_{n+1} r_{n} j_{n} h_{i+1} r_{i}$. Apply $h_{n}$ to cancel $r_{n} j_{n}$, and the result is $R_{n+1}$. Composing these homotopies for all $m>n$ gives a homotopy $R_{n} \sim 1$, on $Z-Y$. The radius of this homotopy (measured in $Y$ ) is less than about $4 \varepsilon_{n}$, so by taking $n$ large enough we get arbitrarily small homotopies pulling $Z-Y$ outside a neighborhood of $Y$.

Finally the local compactness of $Z$ is an elementary consequence of the local compactness of $Y$ and the properness assumption on the $p_{i} h_{i} r_{i}$.

Completion of 2.15. We show that proper $\varepsilon$ domination of the homotopy link, all $\varepsilon>0$, implies reverse tameness. Choose $\varepsilon_{i}$ dominations for $\varepsilon_{i}$ converging to 0 . According to the lemma the mapping cylinder of the holink is strictly rel $Y$ homotopy equivalent to the mapping telescope $Z$. Since $Y \subset X$ is tame, $X$ is strictly rel $Y$ equivalent to the holink homotopy pushout (2.4). Putting these together we get $X \rightarrow X$ and $N \rightarrow Z$, for some neighborhood $N$ of $Y$ in $X$, together with strictly rel $Y$ homotopies of compositions of these to the identities, defined on some neighborhoods of $Y$.

It is not hard to see that such a strict rel $Y$ equivalence of neighborhoods preserves reverse tameness. The key point is that $Z$ is locally compact, so if $U$ is a neighborhood of $Y$ in $Z$ then $Z-U \rightarrow Y$ is proper. The image in $X-Y$ is also proper over $Y$, so lies in the complement of some neighborhood of $Y$. Since-by the lemma- $Y$ is reverse tame in $Z$, it is reverse tame in $X$. 


\section{GeNERAL hOMOTOPICALLY STRATIFIED SETS}

In this section the local homotopy material of $\S 2$ is used to develop the homotopy properties of general (as opposed to ANR, or manifold) homotopically stratified sets. The main structure result is 3.2, on tameness and holinks of pure subsets. Proposition 3.5 characterizes stratified neighborhood deformation retracts in terms of local data. Proposition 3.6 applies to quotients of group actions and is used in the proof of 1.4. Finally 3.7 gives a "pullback" construction which can be used to modify lower skeleta of a homotopically stratified set.

We require all spaces in the section to be metric and will omit this hypothesis from individual statements.

3.1. Definition. A filtered space $X$ is homotopically stratified if for $k>i$, $X^{i}-X^{i-1}$ is tame in $\left(X^{k}-X^{k-1}\right) \cup\left(X^{i}-X^{i-1}\right)$, and the holink of this pair is a fibration over $X^{i}-X^{i-1}$.

The subsets $X_{i}$ of a filtered space $X$ are required to be closed, and as above called "skeleta." Differences $X^{i}-X^{i-1}$ are called "strata." A map $X \rightarrow Y$ preserves strata if every component of a stratum of $X$ has image in a stratum of $Y$. The image stratum is not required to have the same index. A stratumpreserving homotopy is a map $A \times I \rightarrow Y$ with $Y$ filtered, such that each arc $\{a\} \times I$ taken to a single stratum of $Y$. This means that the inverse image filtration of $A \times I$ is the product with $I$ of the filtration of $A \times\{0\}$ or $A \times\{1\}$. Note this definition does not use a preassigned filtration of $A$.

A pure subset $Y$ of a filtered space $X$ is closed, and a union of components of strata. In other words, $Y$ contains each component of $X^{i}-X^{i-1}$ it intersects. For example if a finite group $G$ acts on $M, M / G$ is filtered by orbit type, and $M^{H}$ denotes the subset with isotropy subgroup contained in $H$, then $G M^{H} / G$ is pure in $M / G$.

3.2. Proposition. Suppose $X$ is homotopically stratified and $K \subset X$ is pure. Then there is a nearly (strict and stratum-preserving) deformation retraction of a neighborhood to $K$. Further, holink $(X, K) \rightarrow K$ is a stratified system of fibrations, with respect to the given filtration.

Recall that a deformation is "nearly" something if it satisfies that property on $(0,1]$, that is, until things absolutely have to be mapped into $K$. We recall, from [13], the definition of stratified systems of fibrations. A map $p: E \rightarrow Y$ is a stratified system of fibrations with respect to a filtration of $Y$ if $p$ is a fibration over strata $\left(p: p^{-1}\left(Y^{i}-Y^{i-1}\right) \rightarrow\left(Y^{i}-Y^{i-1}\right)\right.$ is a fibration), and each skeleton is a $p$-NDR. This last means there is a deformation retraction of a neighborhood in $Y$ to $Y_{i}$ which is covered by a deformation retraction of a neighborhood in $E$ to $p^{-1}\left(Y_{i}\right)$.

3.3. Lemma. Stratified systems of fibrations have the approximate lifting property for stratum-preserving homotopies. 
Proof. Suppose we are given a lifting problem

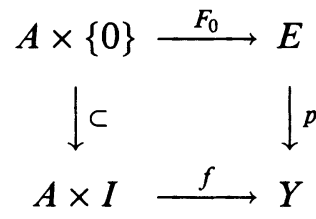

with $p$ a stratified system of fibrations and $f$ stratum-preserving. An approximate lift (see 2.7) is a map $G: A \times I \times(0,1] \rightarrow E$, which is the projection followed by $F_{0}$ on $A \times\{0\} \times(0,1]$, and such that $p G \cup f \times\{0\}$ is continuous.

We actually show that for $\varepsilon: Y \rightarrow(0, \infty)$ there is a similar $\delta$ so that if $B \subset A$ is pure, $f: A \times I \rightarrow Y$ preserves strata within $\delta$ and an exact lift is given over $A \times\{0\} \cup B \times I$, then there is an $\varepsilon$ homotopy of $f$ rel $A \times\{0\} \cup B \times I$ and an extension of the given lift to a lift of this new map.

Here "preserves strata within $\delta$ " means that if $f(a, t) \in Y^{i}$ then the arc $f(a,-)$ is within $\delta$ of $Y^{i}$.

To get an approximate lift from this statement one defines $G$ on $A \times I \times$ $\left\{\frac{1}{i}\right\}_{i=1}^{\infty}$ by choosing $\varepsilon_{i}$ homotopies to maps which lift, for $\varepsilon_{i}$ going to zero. Then fill in between these using the relative version on $A \times I \times\left\{\frac{1}{i+1}, \frac{1}{i}\right\} \subset$ $A \times I \times\left[\frac{1}{i+1}, \frac{1}{i}\right]$.

The proof of the "actual statement" proceeds by induction on the number of skeleta in the filtration. Suppose, therefore, that the statement is known for sets with $n$ or fewer strata.

The first step is to observe that this lifting property implies a relative version. Suppose $j: A \rightarrow[0, \infty)$ is given, $f$ preserves strata within $\delta$, and a lift into $E$ is given on $A \times\{0\} \cup j^{-1}([0,1)) \times I$. We want to conclude that if $\delta$ is small enough there is an $\varepsilon$ homotopy of $f$ rel $A \times\{0\} \cup j^{-1}(0) \times I$ and a lift of the result into $E$ which agrees with the given one on this subset. Define $g:\left(A \times\{0\} \cup j^{-1}([0,1)) \times I\right) \times I \rightarrow Y$ by $g(a, s, t)=f(a, \max \{s+t, 1\})$. Then a lift of this is given over $(-) \times\{0\}$. Extend this to a lift $G$, after small homotopy rel $(-) \times\{0\}$, using the induction hypothesis. The relative lift is given by taking $(a, t)$ to $G(a, s t,(1-s) t)$, where $s=\min \{j(a), 1\}$. The size can be controlled by controlling the homotopy of $g$ and reducing $j$.

For the induction step suppose and $Y$ has exactly $n+1$ strata. Let $N$ be a neighborhood of $Y^{n}$ which has a deformation into $Y^{n}$ covered by a deformation in $E$ into $p^{-1}\left(Y^{n}\right)$. If $\alpha: Y^{n} \rightarrow(0, \infty)$ is small enough then the $2 \alpha$ neighborhood of $Y^{n}$ lies inside $N$. Define $j: A \rightarrow(-\infty, \infty)$ by $j(a)=$ 2 - (distance of $f_{0}(a)$ from $\left.Y^{n}\right) / \alpha$. If $f$ preserves strata within sufficiently small $\delta$ then the restriction to $j^{-1}(-\infty, 1] \times I$ lies in $Y-Y^{n}$, and lifts to $E$ because $p$ is a fibration there. Next apply the deformation of $N$ into $Y^{n}$ to get a homotopy of $f$ to a map which takes $j^{-1}([0, \infty)) \times I$ into $Y^{n}$ and is covered by a homotopy of lifts over $j^{-1}(-\infty, 1] \times I$. If $\alpha$ is sufficiently small this homotopy will also be small. In particular we can arrange the new map $j^{-1}([0, \infty)) \times I \rightarrow Y^{n}$ to preserve strata within any preassigned $\gamma$. Now apply 
the relative lifting property in $Y^{n}$ to extend the lift over $j^{-1}([0, \infty)) \times\{0\} \cup$ $j^{-1}(0) \times I$ to the rest of $A$.

Proof of 3.2. We begin with the case in which $K$ has a single stratum and proceed by induction on the number of strata in $X$. We may assume $K$ is the smallest nonempty skeleton of $X$, since anything smaller must be separated from $K$ and can be deleted. If $X$ has two strata, then the definition of homotopically stratified asserts that $K$ is tame in $X$ and $\operatorname{holink}(X, K) \rightarrow K$ is a fibration. Suppose the same is true for sets with $n$ strata and $X$ has $n+1$. Let $Y \subset X$ be the lowest two strata, so $Y-K$ has only one stratum. $K$ is tame in $Y$ so there is a nearly strict deformation $r: N \times I \rightarrow Y$ of a neighborhood into $K$. $Y-K$ is a single stratum pure subset of $X-K$, so the induction hypothesis implies that holink $(X-K, Y-K) \rightarrow Y-K$ is a fibration. This implies there is a lift of $r: N \times(0,1] \rightarrow Y-K$ to a fiber map of holinks, extending the identity over $N \times\{1\}$.

Proposition 2.9 now applies to show that $K$ is tame in $X$. Further the homotopy link is shown to be, up to $\varepsilon$, a homotopy pushout of fiber maps between homotopy links. The homotopy links are of single strata in sets with $n$ or fewer strata, so are fibrations. The homotopy pushout of fibrations is a fibration, so holink $(X, K) \rightarrow K$ satisfies the $\varepsilon$ lifting property. Since this is so for all $\varepsilon$, it is an approximate fibration. Finally 2.7 implies that a holink which is an approximate fibration is a fibration, completing the proof in this case.

The proof for general $K$ is similar. The "key" step is: Suppose $X \supset K \supset J$ are pure and satisfy

(1) a neighborhood of $J$ in $K$ has a nearly (strict and stratum-preserving) deformation retraction into $K$,

(2) holink $(X-J, K-J) \rightarrow K-J$ is a stratified system of fibrations with the given filtration of $K-J$, and

(3) $K-J \subset X-J$ and $J \subset(X-K) \cup J$ are tame.

The conclusion is that (1) $J \subset K$ is a $p$-NDR subset with respect to $p$ : holink $(X, K) \rightarrow K$, and (2) there is a nearly strict deformation of a neighborhood of $J$ in $X$ into $J$, which is nearly stratum preserving in $K$ and strict with respect to $(X, K)$.

This "key step" follows from the proof of 2.9. Let $r$ denote the deformation assumed to exist in (1). Since it is stratum preserving on $N \times(0,1]$ and the holink in (2) is a stratified system of fibrations, 3.3 gives an approximate lift of $r$ to holinks. The lemma following 2.7 shows there is an exact lift. This is the first part of the hypothesis of 2.9. Next since $K-J \subset X-J$ is assumed to be tame in (3), the first part of the proof of 2.9 applies to extend $r$ to a strict map $R:(X, K) \times I \rightarrow(X, K)$. Composing arcs in $(X, K)$ with $R$ gives a deformation of holink $(X, K)$, and in particular a deformation of $p^{-1}(N)$ into $p^{-1}(J)$ which covers the deformation $r$. This proves conclusion (1). Second since $J \subset(X-K) \cup J$ is assumed tame, 2.9(1) asserts that $J \subset X$ is tame. 
More precisely the proof shows that there is a nearly strict deformation $S^{*}$ of a neighborhood which satisfies conclusion (2).

Now we show the proposition follows from the "key step." Suppose the proposition is known for sets with at most $n$ distinct strata, and suppose $X$ has $n+1$. Suppose $J \subset X$ is pure and we want to find a nearly stratum-preserving deformation retraction of a neighborhood to $J$. Let $K$ be the next-to-the-top skeleton of $X$. Then $K$ and $(X-K) \cup J$ are homotopically stratified with $n$ or fewer strata, so the hypotheses of the key step follow from the induction hypothesis. Conclusion (2) of the key step gives a deformation which is nearly stratum-preserving in $K$ and nearly strict in $(X, K)$. Since there is only one level of strata not in $K$, this means the deformation is nearly stratum-preserving in $X$.

Similarly suppose $X$ has $n+1$ strata, and we want to show $p: \operatorname{holink}(X, K)$ $\rightarrow K$ is a stratified system of fibrations. The single stratum case implies $p$ is a fibration over the strata of $K$, so it remains to see that the skeleta $J \subset K$ are $p$-NDR subsets. This is conclusion (1) of the key step, which applies since the induction hypothesis implies the hypotheses of the key step.

Proposition 3.5 characterizes "neighborhood deformation retracts" in the category, in terms of the local data. The main application is to boundaries in manifold homotopically stratified sets. The global version is useful in recognizing "h-cobordisms."

3.4. Definition. A closed subset $V \subset X$ is homotopically transverse to $Y \subset$ $X$ if the inclusion $\operatorname{holink}(V, V \cap Y) \rightarrow \operatorname{holink}((X-Y) \cup V, V \cap Y)$ is a fiber homotopy equivalence over $V \cap Y$. We note that by part (2) of the corollary to 2.4 , holink $((X-Y) \cup V, V \cap Y)$ is the restriction to $V \cap Y$ of holink $(X, Y) \rightarrow Y$. The definition can therefore be generalized to strict maps $(V, W) \rightarrow(X, Y)$ by requiring that the natural map from holink $(V, W)$ to the pullback of holink $(X, Y)$ be a fiber homotopy equivalence.

If $X$ is a homotopically stratified set then we can consider $Y \subset X$ homotopically transverse to the skeleta of $X$. If $Y$ is homotopically stratified with respect to the induced filtration then the formulae for the holinks of skeleta show that this condition can be recognized locally. It is equivalent to: if $A$ and $B$ are strata of $X$, and $B$ is in the closure of $A$, then $Y \cap(A \cup B)$ is homotopically transverse to $B \subset A \cup B$.

A stratum-preserving map $X \rightarrow Y$ of homotopically stratified sets is homotopically transverse (to the given filtration) in this sense if and only if the mapping cylinder with the natural filtration is a homotopically stratified set. This condition is an analog of the "transverse" maps of [4].

3.5. Proposition. Suppose $X$ is homotopically stratified and $Y \subset X$ is closed. Then there is a stratum-preserving deformation retraction of a neighborhood in $X$ to $Y$ if and only if $Y$ is homotopically stratified with respect to the induced 
filtration, the inclusion $Y \subset X$ is homotopically transverse, and

$$
Y \cap\left(X^{i}-X^{i-1}\right) \subset\left(X^{i}-X^{i-1}\right)
$$

is a neighborhood deformation retract, for each $i$.

This deformation is genuinely stratum-preserving, not "nearly" as in 3.2. There is also a global version: $X$ itself deformation retracts if and only if $Y$ is homotopically transverse, and $\left(X^{i}-X^{i-1}\right)$ deformation retracts to $Y \cap$ $\left(X^{i}-X^{i-1}\right)$ for each $i$.

Proof. The "only if" direction is elementary. For the converse we proceed by induction, and assume there is $R_{i}: X_{i} \times I \rightarrow X_{i}$ stratum-preserving, the identity on $X_{i} \times\{1\} \cup\left(Y \cap X_{i}\right) \times I$, and there is a neighborhood $N$ of $Y \cap X_{i}$ such that $R_{i}(N \times\{0\}) \subset Y$. We want to extend this to $R_{i+1}$ defined on $X_{i+1}$. For notational convenience we set $X=X_{i+1}, Y=Y \cap X_{i+1}$.

The first step is to observe that since $R_{i}$ is stratum-preserving and holink $\left(X, X_{i}\right)$ is a stratified system of fibrations (3.2), there is a fiber homotopy of the holink covering $R_{i}$, and fixed over $Y \cap X_{i}(3.3,2.7)$. Further since $Y$ is homotopically transverse we may assume that over $N \times\{0\}$ the holink maps into holink $\left(Y, Y \cap X_{i}\right)$. We use this holink information to extend $R_{i}$ to a neighborhood of $X_{i}$, using an analog of 2.2 for pairs. For this we need to show that $\left(X_{i}, Y \cap X_{i}\right)$ is "relatively tame" in $(X, Y)$, by which we mean there is a nearly strict deformation retraction of a neighborhood to $X_{i}$, which keeps $Y$ in $Y$.

Since $X_{i}$ is tame there is $T: X \times I \rightarrow X$ nearly strict which deforms a neighborhood into $X_{i}$, but does not preserve $Y$. To fix this we suppose $S: X \times$ $I \rightarrow X$ is nearly strict, preserves $Y$, is the identity on $X_{i}$, and pushes into $Y \cap X_{i}$ a set of the form (neighborhood of $\left.Y \cap X_{i}\right) \cap(\delta$ neighborhood of $Y$ ), where $\delta: Y \rightarrow[0, \infty)$ has $\delta^{-1}(0)=Y \cap X_{i} . S$ is obtained by following a deformation of a $2 \delta$ neighborhood in $X-Y$ into $Y-Y \cap X_{i}$ (obtained from the deformation hypothesis on strata), with a nearly strict deformation of a neighborhood in $Y$ into $Y \cap X_{i}$ (obtained from tameness in $Y$ ). We now choose functions $a, b: X \rightarrow[0,1]$ and define a deformation by $T(S(x, s), t)$, where $(s, t)$ lies on the line from $(1,1)$ to $(a(x), b(x))$. If $a b=0, b=1$ on $Y$, and $a=1$ on the complement of the $\delta$ neighborhood of $Y$ then this defines a relative nearly strict neighborhood deformation retraction, as required. We note that $a, b$ satisfying these conditions must be discontinuous at $Y \cap X_{i}$, but the composition extends continuously to be the identity there.

The adjoint of the relative deformation above, and the distance from $X_{i}$, define a map from a neighborhood of $X_{i}$ to the mapping cylinder of $\operatorname{holink}\left(X, X_{i}\right)$ $\rightarrow X_{i}$. This map is the identity on, and strict with respect to, $X_{i}$ and takes the intersection with $Y$ into the mapping cylinder of holink $\left(Y, Y_{i}\right)$. Compose this with the map induced by the fiber homotopy of holinks covering $R_{i}$ constructed at the beginning of the proof. Follow this with the evaluation map, from the mapping cylinder of $\operatorname{holink}\left(X, X_{i}\right) \rightarrow X_{i}$, to $\left(X_{i+1} ; Y \cap X_{i+1}, X_{i}, Y \cap X_{i}\right)$. This 
three-fold composition gives an extension of $R_{i}$ to a neighborhood of $X_{i}$ in $X$, which is strict with respect to $X_{i}$ and deformation retracts the intersection with a neighborhood of $Y$, into $Y$.

Once $R_{i}$ is appropriately extended to a neighborhood of $X_{i}$ it is easy to combine it with a deformation retraction of a neighborhood in $X-X_{i}$ to $Y-Y_{i}$, to get an appropriate deformation $R_{i+1}$.

Next, "branched covers" and group actions. We define a stratum-preserving map of filtered spaces $q: X \rightarrow Y$ to be a multiply branched cover if it is open, and for each $i ; q^{-1}\left(Y^{i}-Y^{i-1}\right) \rightarrow Y^{i}-Y^{i-1}$ is a covering space, and $q^{-1}\left(Y^{i}\right) \rightarrow Y^{i}$ has the unique lifting property for strict paths in $\left(Y^{i}, Y^{i-1}\right)$. See the explanations following 2.6 for definitions of these terms.

\subsection{Proposition. Suppose $X$ and $Y$ are finite dimensional.}

(1) If $q: X \rightarrow Y$ is a multiply branched cover over a filtration of $Y$, then $Y$ is a homotopically stratified set if and only if $X$ with the induced filtration is a homotopically stratified set.

(2) If $G$ is a discrete group which acts on a locally connected filtered metric space $X$ so that isotropy subgroups are locally constant on strata, and $N(H) / H$ acts properly discontinuously on the set of points with isotropy subgroup $H$, then the quotient $q: X \rightarrow X / G$ is a multiply branched cover over the induced filtration of $X / G$.

Proof. It is not hard to see that a stratum-preserving open map of filtered metric spaces which is a covering on each stratum is "strongly light" in the sense of 2.6. Proposition 2.6 therefore applies to pairs of strata and shows that tameness in $X$ is equivalent to tameness in $Y$ or $X / G$. Further, a pullback over a covering space is a fibration if and only if the original map is one. Therefore the holink formula of 2.6 shows the holink fibration condition in $X$ is equivalent to the condition in $Y$ or $X / G$. Together these imply (1).

For the second statement, note that a quotient map is automatically open. The covering space property is equivalent to the condition that $N(H) / H$ act properly discontinuously (where $N(H)$ denotes the normalizer of $H \subset G$ ). This leaves the path lifting property to be verified.

The quotient map is strongly light, as above. This can be improved with the local connectivity assumption: Suppose $U$ is a neighborhood of $x \in X$ with the local light property, containing no other preimage of $q(x)$ and separated from $q^{-1} q(U)-U$. Let $V$ be the connected component of $U$ containing $x$, then $V$ is also a neighborhood and if $g \in G$ either $g V=V$ or $g V$ is disjoint from $V$. Further, if $g V=V$ then $g x=x$.

Now consider the strict lifting property. Suppose

$$
f:([0,1], 0) \rightarrow\left(X^{i} / G, X^{i-1} / G\right)
$$

is strict, and a lift of $f(1)$ to $X^{i}$ is given. Since $\left(X^{i}-X^{i-1}\right) \rightarrow\left(X^{i}-X^{i-1}\right) / G$ is a covering space there is a lift $F:(0,1] \rightarrow\left(X^{i}-X^{i-1}\right)$ extending this. We need 
to see that $F$ extends continuously to $[0,1]$. Choose some $c$ in the preimage of $f(0)$ and a neighborhood $V$ of $c$ with the separation property of the previous paragraph. Let $t$ be small enough that $f((0, t]) \subset q(V)$, and choose a lift $\widetilde{F}$ of $f$ on $(0, t]$ which intersects $V$. There is some $g$ so that $F=g \widetilde{F}$ on $(0, t]$, and we show that $F$ extends continuously by $F(0)=g c$. Let $W$ be an arbitrarily small neighborhood of $g c$, inside $g V$ and also satisfying the separation property. Choose $s$ so that $f(0, s] \subset q(W)$ and a lift $\widehat{F}$ on $(0, s]$ which intersects and therefore is contained in $W$. Let $h \in G$ be so that $F=h \widehat{F}$. Since $\widehat{F}$ and $F$ both intersect $V, h V=V$ and $h(g c)=g c$. But this implies that $h W=W$, so $F(0, s] \subset W$. Since $F$ is eventually in every neighborhood of $g c$, it extends continuously. This verifies the lifting property, so completes the proof of the proposition.

The final result of the section describes a way to modify lower skeleta. The $W, X, Y$, etc. in the statement are understood to be homotopically stratified sets.

3.7. Pullback Lemma. Suppose $Y \subset X$ is a pure subset. and $f: Z \rightarrow Y$ is a stratum-preserving map. Then there is a stratum-preserving $F: W \rightarrow X$ so that $F^{-1}(Y)=Z$ and $F$ restricted to $Z$ is $f$, and for each stratum component $A$ of $X$ not in $Y, F: F^{-1}(A) \rightarrow A$ is a homotopy equivalence and holink $\left(F^{-1} \bar{A}, F^{-1} \delta A\right)$ is fiber homotopy equivalent to the pullback of holink $(\bar{A}, \delta A)$.

Proof. The construction of $W$ is nearly specified by the statement of the lemma. Proceed by induction on skeleta, and assume $F^{n-1}:\left(W^{n-1} \cup Z\right) \rightarrow$ $\left(X^{n-1} \cup Y\right)$ is defined. Let $E \rightarrow\left(W^{n-1} \cup Z\right)$ be the pullback of $\operatorname{holink}\left(X^{n} \cup Y, X^{n-1} \cup Y\right) \rightarrow\left(X^{n-1} \cup Y\right)$. Composition defines a map $E \rightarrow$ holink $\left(X^{n} \cup Y, X^{n-1} \cup Y\right) \rightarrow\left(X^{n}-X^{n-1} \cup Y\right)$. Define $W^{n} \cup Z$ to be the homotopy pushout of

$$
\left(X^{n}-X^{n-1} \cup Y\right) \leftarrow E \rightarrow\left(W^{n-1} \cup Z\right) .
$$

By tameness there is a strict rel $X^{n-1} \cup Y$ homotopy equivalence-from the homotopy pushout of

$$
\left(X^{n}-X^{n-1} \cup Y\right) \leftarrow \operatorname{holink}\left(X^{n} \cup Y, X^{n-1} \cup Y\right) \rightarrow\left(X^{n-1} \cup Z\right)
$$

to $X^{n} \cup Y$. Composing this with the natural map of pushouts gives the map $F^{n}$ required for the induction step.

\section{ANR HOMOTOPICALLY STRATIFIED SETS}

An ANR (Absolute Neighborhood Retract) is a space which when embedded as a closed subspace of a normal space, has a neighborhood which retracts to it. There are homological characterizations (4.1) of tameness and fibrations in this 
context, so ANR homotopically stratified sets are relatively easy to recognize and manipulate. Proposition 4.2 gives a recognition criterion which is the basis for the proof of Proposition 1.5. Lemma 4.3 replaces top strata by polyhedra, and provides the contact with PL versions of finiteness and simple homotopy. Control Lemma 4.4 establishes connections with the controlled theory of [13].

Much of the section involves "layered sets" (defined in 4.4), roughly ANR homotopically stratified sets with reverse tame skeleta. Theorem 4.7 characterizes these up to stratified homotopy and implies Theorem 1.11. "Whitehead torsion" is developed in this context in 4.8 , and the proof of the simple homotopy Theorem 1.10 is given there and in 4.10.

As in the previous section, all spaces are required to be metric.

A map $p: E \rightarrow X$ is said to have locally constant homology if each point has neighborhoods $U \supset V$ with the following property: If $A \subset V$ is a neighborhood of a point $y$, then there is a neighborhood $B \subset A$ such that

$$
\text { image }\left(H_{i}\left(p^{-1} B\right) \rightarrow H_{i}\left(p^{-1} A\right)\right) \rightarrow \operatorname{image}\left(H_{i}\left(p^{-1} V\right) \rightarrow H_{i}\left(p^{-1} U\right)\right)
$$

is an isomorphism, for all $i$. Locally constant fundamental group and $\mathbf{Z} \pi_{1}$ coefficient homology are defined similarly, as above, using the indicated functors instead of $H_{i}$.

4.1. Lemma. Suppose $p: E \rightarrow X$ is given and $X$ is an ANR. If $p$ has the approximate lifting property for polyhedra then it has locally constant fundamental group and $\pi_{1}$ coefficient homology. If $X$ is finite dimensional the converse is true, and further if $E$ is the homotopy link of $X$ in an ANR then $p$ is a fibration.

Proof. First the easy direction, that the lifting property implies locally constant homology. If $U$ is given, let $V \subset U$ be a neighborhood which contracts to $x$ inside $U$. Similarly if a neighborhood $A \subset V$ of a point $y$ is given, choose B which contracts to $y$ inside $A$. Then there is a contraction of $V$ to $y$ inside $U$, which keeps $B$ inside $A$. To show that the homomorphism of images is injective we suppose $c$ is an element in the chain group $C_{i}\left(p^{-1} B\right)$ with $\partial c=0$ and suppose the homology class of $c$ is trivial in $p^{-1} V$. We want to show the homology class is trivial in $p^{-1} A$. Triviality means there is $d \in C_{1+1}\left(p^{-1} V\right)$ with $\partial d=c$. Apply the deformation above to get a homotopy of $p d$ to the constant map at $y$, such that $p c$ stays in $A$. Apply the approximate lifting property to this homotopy to get a homotopy of $c$ in $p^{-1} A$ to $c^{\prime}=\partial d^{\prime}$ inside $p^{-1} B$. The homotopy and $d^{\prime}$ give a chain in $C_{i}\left(p^{-1} A\right)$ with $c$ as boundary. Surjectivity is similar.

For the converse we suppose $\pi_{1}$ and the homology are constant over $U \supset V$ and take covers so the local fundamental groups are trivial. Let $p^{*}: E^{*} \rightarrow$ $V$ be $p^{-1} V \rightarrow V$ converted into a fibration. The fibration has the lifting property for any space, so it is sufficient to show that the pair $\left(E^{*}, p^{-1} V\right)$ is $(\delta, k)$-connected over $V$ in the sense of [13 I, 5.1]. 
The inclusion $p^{-1} V \rightarrow E^{*}$ is a fiber map over $V$ so induces a morphism of homology Leray spectral sequences $H_{i}\left(V ; H_{j}\left(p^{-1} v\right)\right) \rightarrow H_{i}\left(V ; H_{j}\left(p^{*-1} v\right)\right)$. These spectral sequences are defined and converge since $X$ is finite dimensional. The inclusion is a homotopy equivalence so induces an isomorphism of abutments. The coefficients in both sequences are constant, so the comparison theorem for spectral sequences implies the morphism of coefficients is an isomorphism. Conversely if $K \subset V$ is open then the Leray sequences over $K$ have isomorphic $E_{2}$ terms so $H_{i}\left(p^{-1} K\right) \rightarrow H_{i}\left(p^{*-1} K\right)$ is an isomorphism. This means the identity map of the pair $\left(p^{*-1} K, p^{-1} K\right)$ is relatively homologically $(\delta, k)$-trivial in the sense of [13 I, 5.1] for all $k$ and $\delta>0$. But according to [13 I, 5.2] this implies the pair is $(\delta, k)$-connected, as required.

Finally we show that if $X \subset Z$ is a tame pair of ANRs and holink $(Z, X) \rightarrow$ $X$ has the $\varepsilon$ lifting property for complexes, all $\varepsilon>0$, then it is actually a fibration. It is shown in 2.7 that the $\varepsilon$ lifting property for arbitrary spaces implies it is a fibration, so we need to see this property for complexes implies it for spaces. As in 2.7 consider the adjoint; a map $K \rightarrow \operatorname{holink}(Z, X)$ corresponds to a strict map $(K \times[0,1], K \times\{0\}) \rightarrow(Z, X)$. It is a property of ANRs that such a map factors within any $\varepsilon$ through a complex. To see this, extend the map to a map of linear spaces in which $K, Z$ embed, use the ANR property of $Z$ to retract a neighborhood to $Z$, and approximate the preimage open set in the linear space containing $K$ by a complex. This can be done to preserve strictness, using the tameness of $X$, and applies also to a homotopy $K \times I \rightarrow X$. We can therefore approximately factor an arbitrary lifting problem through one for complexes, and use the lifting property for complexes to get an approximate solution to the original problem.

The next result applies this when local fundamental groups are trivial. The definition of homotopically stratified set involves conditions on pairs of strata with intermediate strata deleted; one considers $\left(X^{n}-X^{n-1}\right) \cup\left(X^{i}-X^{i-1}\right) \supset$ $\left(X^{i}-X^{i-1}\right)$. The point here is that these intermediate strata need not be deleted; we can consider $\left(X^{n}-X^{i-1}\right) \supset\left(X^{i}-X^{i-1}\right)$. In a homotopically stratified set the holink of this pair is a fibration (by 3.2 a stratified system of fibrations, over a single stratum). The proposition provides a partial converse.

4.2. Proposition. Suppose $X$ is a finite dimensional $A N R$ and has an $A N R$ filtration. Suppose the next-to-top skeleton is homotopically stratified and 1-LC in $X$. Then $X$ is homotopically stratified if and only if $\operatorname{holink}\left(X-X^{i-1}, X^{i}-\right.$ $\left.X^{i-1}\right) \rightarrow\left(X^{i}-X^{i-1}\right)$ is a fibration, for all $i$.

Proof. As remarked above the "only if" part follows from 3.2. Assume the converse is known for skeleta with $k$ or fewer strata, and suppose $X$ has $k+1$. Let $X^{i}$ be the smallest skeleton. Then the induction hypothesis applies to $\left(X-X^{i}\right) \supset\left(X^{n}-X^{i}\right)$, so it remains to show $\left(X-X^{n}\right) \cup X^{i} \supset X^{i}$ is tame and has holink a fibration. This pair has trivial local fundamental group, so is tame 
by 2.12 . The holink, by $2.9(3)$, fits in a homotopy pushout diagram over $X^{i}$ :

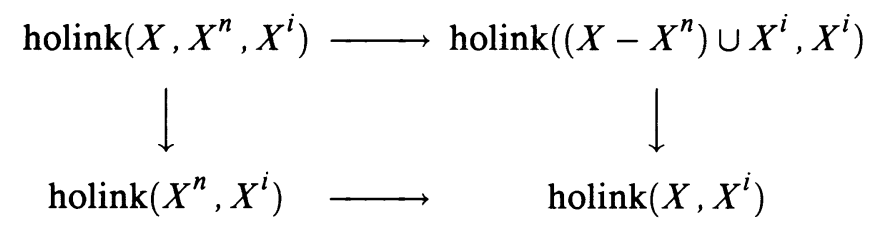

The lower left space is fibered over $X^{i}$ since $X^{n}$ is homotopically stratified, the lower right space is fibered by hypothesis. The main step in the proof is that the triple holink in the upper left çorner is a fibration over $X^{i}$. Unfortunately the fact that three of the spaces are fibered does not in general imply that the remaining one, in the upper right corner, is fibered. However since they are fibered they have locally constant homology, and this does imply that holink $\left(\left(X-X^{n}\right) \cup X^{i}, X^{i}\right)$ has locally constant homology over $X^{i}$. The 1-LC hypothesis implies that it has trivial local fundamental group. Lemma 4.1 in this case implies it is fibered over $X^{i}$, so $X$ is homotopically stratified.

We begin the main step, showing that $\operatorname{holink}\left(X, X^{n}, X^{i}\right) \rightarrow X^{i}$ has the $\varepsilon$ lifting property for complexes. Suppose a lifting problem

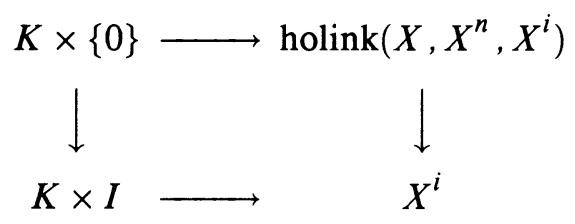

is given. The map into the triple holink is adjoint to a map $K \times\{0\} \times I \times$ $I \rightarrow X$. Consider the intermediate lifting problem into $\operatorname{holink}\left(X^{n}, X^{i}\right)$, which corresponds to extending the restriction $K \times\{0\} \times I \times\{0\} \rightarrow X^{n}$. If there is a stratum-preserving lift in the sense that the adjoint $K \times I \times I \times\{0\} \rightarrow$ $X^{n}$ preserves strata with respect to the first $I$ coordinate, then there is lift into the triple holink. According to 2.9(2) the triple holink (or rather the fiber homotopically equivalent controlled version) is $\varepsilon$ equivalent to the pullback of holink $\left(X-X^{i}, X^{n}-X^{i}\right) \rightarrow X^{n}-X^{i}$. Thus it is sufficient to lift the restriction $K \times I \times\{1\} \times\{0\} \rightarrow X^{n}-X^{i}$ into holink $\left(X-X^{i}, X^{n}-X^{i}\right)$. But the restriction is still stratum-preserving, and the holink is a stratified system of fibrations since $X-X^{i}$ is homotopically stratified. Therefore by 3.3 the lift exists.

We now consider stratum-preserving lifts of homotopies into holink $\left(X^{n}, X^{i}\right)$. Suppose $K \times\{0\} \rightarrow \operatorname{holink}\left(X^{n}, X^{i}\right)$ is given. This can be arbitrarily closely approximated by a map whose adjoint $F_{0}: K \times\{0\} \times I \rightarrow X^{n}$ has subcomplexes as inverse images of skeleta in $X^{n}$. Assume there is a stratum-preserving extension $F^{k}: F_{0}^{-1}\left(X^{k}\right) \times I \times I \rightarrow X^{k}$. Since it is stratum-preserving and $X^{n}$ is homotopically stratified it is covered by a fiber map of $\operatorname{holink}\left(X^{k+1}, X^{k}\right)$. $F_{0}^{-1}\left(X^{k}\right) \subset F_{0}^{-1}\left(X^{k+1}\right)$ is tame because it is a subcomplex. Corollary 2.3 therefore implies that $F^{k}$ extends to a stratum-preserving $F^{k+1}$. By induction there is a stratum-preserving lift on all of $K$. 
The next lemma replaces the top stratum by a polyhedron. When $X$ is a compact layered set, the corollary to 4.7 shows that all strata can be replaced in this way.

4.3. Stratum Replacement Lemma. Suppose $X$ is a locally compact finite dimensional ANR with a tame filtration and $X^{n}=X$. Then there is another one, $Y$, with $X \subset Y$ and $r: Y \rightarrow X$ a stratified retraction such that $Y^{n-1}=X^{n-1}$, $Y^{n}-Y^{n-1}$ is a flat manifold mapping cylinder neighborhood of $X^{n}-X^{n-1}$, and $r$ is the mapping cylinder retraction.

Proof. Since $X^{n}-X^{n-1}$ is a locally compact finite dimensional ANR, there is a proper embedding in $\mathbf{R}^{k}$, some $k$, with a mapping cylinder neighborhood (see e.g. [13 I, §3.1]). Denote this neighborhood by $U$, with retraction $r: U \rightarrow$ $\left(X^{n}-X^{n-1}\right)$. Define $Y$ to be $X^{n-1} \cup U$, and extend $r$ by the identity on $X^{n-1}$. Topologize this with the smallest topology such that the inclusions and $r$ are continuous.

Continuity of $r$ implies continuity of the mapping cylinder deformation retraction of $Y$ to $X$. This shows the inclusion is a stratum-preserving homotopy equivalence. Since such equivalences preserve tameness, this implies $Y$ is as required.

Now the connection between strict homotopy and the " $\varepsilon$ control" used in [13].

4.4. Control Lemma. Suppose $Z$ is a metric space, $A$ is compact, and $f$, $g:(A, B) \rightarrow($ cone $Z, Z)$ are closed, strict, and agree on $B$. Then given any strict $\varepsilon:($ cone $Z, Z) \rightarrow([0,1],\{0\})$ there is a radial homeomorphism $r$ of cone $Z$ so that $d(r f(x), r g(x))<\varepsilon(r f(x))$.

Here "radial" means $r(z, t) \in\{z\} \times I$, so in particular $r$ is the identity on $Z \times\{1\}$ and $r(0)=0$. Homotopies provide a particularly useful special case. Suppose $h:(A \times I, B \times I) \rightarrow($ cone $Z, Z)$ is strict, proper, and has radius 0 on $B$ (i.e. is constant on $B$ ). Then we can radially reparameterize the cone to get a homotopy which has radius less than any given $\varepsilon$. The definition of "radius less than $\varepsilon$ " for a homotopy is that it is within $\varepsilon$ of the projection $A \times I \rightarrow A$ followed by $h_{0}: A \rightarrow$ cone $Z$. Since $h$ and this map agree on $B \times I$, the lemma applies.

Proof. Assume $Z$ is compact (replace it with the union of the images of $f$ and $g$ if not). Using compactness one finds a strict $\alpha:([0,1],\{1\}) \rightarrow([0,1],\{0\})$ such that $\varepsilon(z, t)>\alpha(t)$. Since $d(f, g)=0$ when $f(x) \in Z$ (i.e. when $x \in B$ ) there is a strict $\beta:([0,1],\{1\}) \rightarrow([0,1],\{0\})$ so that if $f(x)=(\hat{f}, t)$ then $d(f(x), g(x))<\beta(t)$. The objective is now to find a homeomorphism $r: I \rightarrow I$ so that $f(x)=(\hat{f}, t)$ and $g(x)=(\hat{g}, u)$ then $d((\hat{f}, r(t)),(\hat{g}, r(u))<\alpha(t)$.

The result is independent of the metric on the cone. For the next step we use the metric given by $d((x, s),(y, t))=\sqrt{s^{2}+t^{2}-2 s t \cos (\min \{d(x, y), \pi / 2\}}$. If 
$Z \subset S^{n}$ and has diameter less than $\pi / 2$ then this is the metric obtained by considering the cone as a subset of $D^{n+1}$. The properties we use are that if $s>t$ then $d((x, s),(y, s))>d((x, t),(y, t))$, and $d((x, s),(x, t))=s-t$.

Divide $[0,1]$ into consecutive intervals $J_{i}, i=1, \ldots, \infty$ so that there are $\alpha_{i}>0$ such that the diameter of $J_{i}$ is less than $\alpha_{i}$ and $\alpha>4 \alpha_{i}$ on $J_{i-1} \cup J_{i} \cup J_{i+1}$. Then choose intervals $K_{i}, i=1, \ldots, \infty$, so that if $i>1$, $K_{i}>J_{i}$ and $\beta<\alpha_{i}$ on $K_{i}$.

Choose a homeomorphism $r$ of $I$ which takes $K_{i}$ to $J_{i}$. Denote $f(x)$ and $g(x)$ by $(\hat{f}, t)$ and $(\hat{g}, u)$ as above, and let $i$ be such that $t \in K_{i}$. Then $d((\hat{f}, r(t)),(\hat{g}, r(u))) \leq d((\hat{f}, r(t)),(\hat{g}, r(t)))+d((\hat{g}, r(t)),(\hat{g}, r(u))) \leq$ $\beta(t)+|r(t)-r(u)| \leq 4 \alpha_{i}<\alpha(t)$. Therefore this $r$ satisfies the conclusions of the lemma.

Next, we develop the objects used in stratified simple homotopy theory.

4.5. Definition. $X$ is a layered set if it is a locally compact locally finite dimensional metric space, is a homotopically stratified set with ANR strata, and for each $n, X^{n} \subset X^{n+1}$ is reverse tame.

Remark. It seems likely there is a homological recognition principle for these. Specifically, if $X \supset Y$ we say $X$ has locally constant homology near $Y$, if each point in $Y$ has neighborhoods $U \supset V$ in $X$ with the following property: If $A \subset V$ is a neighborhood in $X$ of a point $y \in Y$ then there is a neighborhood $B \subset A$ such that

$$
\operatorname{image}\left(H_{i}(B-Y) \rightarrow H_{i}(A-Y)\right) \rightarrow \operatorname{image}\left(H_{i}(V-Y) \rightarrow H_{i}(U-Y)\right)
$$

is an isomorphism for all $i$. If $V \subset X$ has constant local fundamental group near $Y$ in the sense of 2.11 then it has locally constant $Z \pi_{1}$ coefficient homology if the branched cover of $V$ corresponding to the kernel of the homomorphism to $\pi_{1} V \rightarrow \pi$ has locally constant homology.

We then speculate that a locally compact finite dimensional filtered space $X$ with ANR strata is layered if and only if $\left(X^{k}-X^{k-1}\right) \cup\left(X^{i}-X^{i-1}\right)$ has locally constant fundamental groups and $\mathbf{Z} \pi_{1}$ coefficient homology near $X^{i}-X^{i-1}$, for all $k>i$.

Locally constant $\mathbf{Z} \pi_{1}$ homology hopefully implies homological tameness, so $X^{i}-X^{i-1}$ should be tame by 2.11 . This in turn means $\left(X^{k}-X^{k-1}\right) \cup\left(X^{i}-X^{i-1}\right)$ is strictly and rel $Y$ homotopy equivalent to the homotopy pushout of the holink diagram, as in 2.2. This should imply that the holink satisfies the local constancy condition of 4.1, so it is a fibration and $X$ is homotopically stratified. Finally, reverse tameness might follow from 2.14 and the eventual Hurewicz theorem of [13 I, $\S 5]$, or perhaps more simply from 4.5 , below.

The next lemma is a key step in the construction of layered sets. 
4.6. Lemma. Suppose $p: E \rightarrow X$ is a stratified system of fibrations over a locally compact finite dimensional ANR. Then the following are equivalent:

(1) There is $n$ so that for every $\varepsilon: X \rightarrow(0, \infty), E$ is $\varepsilon$ dominated by an $n$-dimensional proper polyhedron over $X$.

(2) $p: E \rightarrow X$ is fiber homotopy equivalent to $\operatorname{holink}(Z, X)$ for some locally compact $Z \supset X$ in which $X$ is tame and reverse-tame, and with $Z-X$ a finite dimensional polyhedron.

(3) Each fiber $p^{-1}(x)$ is dominated by a finite complex.

Part (2) can be sharpened a little: In one direction, it is sufficient to have $Z$ with $Z-X$ a finite dimensional ANR, since it can be replaced by a polyhedron by the Replacement Lemma 4.3. In the other direction, if $p$ satisfies the other two conditions then the $Z$ we construct is proper over $X$ and strictly and rel $X$ homotopy equivalent to the mapping cylinder of $p$. This is a bit stronger than saying $Z$ has the right holink.

(1) implies (2). Choose $\varepsilon_{i} \rightarrow 0$, and proper $\varepsilon_{i}$ dominations $\left(K_{i}, r_{i}, j_{i}, h_{i}\right)$, of $E$ by polyhedra. According to Lemma 2.16, the mapping telescope is strictly rel $X$ homotopy equivalent to $\operatorname{cyl}(p)$, and $X$ is tame and reverse-tame in it. Closely approximating the maps $j_{i+1} r_{i}$ used in the construction of the telescope by PL maps yields a space with the same properties, and with $Z-X$ a polyhedron.

(2) implies (1). The proof of 2.15 shows that $E$ can be properly $\varepsilon$ dominated by a space of the form $N-U$, where $N$ is a closed and $U$ is an open neighborhood of $X$ in $Z$. If $Z-X$ is a polyhedron these may be chosen PL, so this provides a domination of $E$ by a proper polyhedron of the dimension of $Z-X$.

(1) implies (3). Suppose $Y \subset X$ is a closed $p$-NDR subset, then we show that the restriction $E \mid Y \rightarrow Y$ is also $\varepsilon$ dominated in the sense of (1). Let $U$ be a neighborhood of $Y$ which $p$-deforms to it. Choose $\delta<\varepsilon / 4$ and small enough so that the $2 \delta$ neighborhood of $Y$ is contained in $U$, and on this the deformation has radius less than $\varepsilon / 2$. Choose $E \rightarrow K \rightarrow E$ a $\delta$ domination as in (1). $E \mid Y$ maps into the restriction to the $\delta$ neighborhood $K \mid Y^{\delta}$, which then maps into $E \mid Y^{2 \delta} . E \rightarrow K$ is proper and $Y$ is closed, so there is a closed PL subset $K \subset L \subset E \mid Y$. Since there is an $\varepsilon / 2$ deformation retraction of $U$ to $Y$ covered by a deformation of $E$, there is an $\varepsilon / 2$ retraction $E\left|Y^{2 \delta} \rightarrow E\right| Y$. Putting these together gives an $\varepsilon$ retraction of $L$ to $E \mid Y$.

Now let $Y$ be a discrete set with one point in each stratum component of $X$. This is a $p$-NDR because the skeleta are, and the strata (which are ANRs) are locally contractible. The restriction to this $Y$ is the disjoint union of the fibers of $E$, so by the argument above these are dominated. 
(3) implies (1). This proceeds by induction on the number of strata, so we suppose the restriction $E \mid X^{n-1}$ has proper PL $\varepsilon$ dominations of bounded dimension and show the same is true of $E \mid X^{n}$. Since this only involves the $n$-skeleton we assume $X=X^{n}$.

We may assume the top stratum of $X$ is a polyhedron. If not, apply Lemma 4.3 to obtain a retraction from a filtered set with PL top stratum to $X$. Pull $E$ back to this, then if $\delta$ is small enough, a proper $\delta$ domination of the pullback is a proper $\varepsilon$ domination of $E \mid X$.

According to the definition of a stratified system of fibrations, $X^{n-1}$ is a $p$-NDR. This means that if $\varepsilon>0$ there is an $\varepsilon$ homotopy $h: X \times I \rightarrow X$ which is the identity on $X \times\{0\}$ and $X^{n-1} \times I$, there is an open neighborhood $N$ of $X^{n-1}$ so that $h_{1}(N) \subset X^{n-1}$, and $h$ is covered by a homotopy $H: E \times I \rightarrow E$. Denote the frontier (closure of $N$ intersect $X-N$ ) by $M$, and choose $N$ so that $X-N$ is a compact polyhedron and $M$ is collared in $N$.

Let $Y$ denote the homotopy pushout of $X^{n-1} \leftarrow M \rightarrow X-N$, where the first map is $h_{1}$ and the second the inclusion. Then $h_{1}$ factors through $Y$. Similarly $H_{1}$ factors through the pushout of $E\left|X^{n-1} \leftarrow E\right| M \rightarrow E \mid(X-N)$, which we denote by $F$. This shows that $F \varepsilon$-dominates $E$. It will therefore be sufficient to produce arbitrarily small proper PL dominations of $F$. For this it is sufficient to find dominations of the pair $(E|(X-N), E| M)$; the induction hypothesis gives dominations of $E \mid X^{n-1}$, and the pushout of dominations gives a domination of the pushout. Note that if the component spaces in a pushout are PL then the maps can be arbitrarily closely approximated by PL maps, to give a PL pushout.

The situation is now that we have a genuine fibration with dominated fiber over a compact PL pair, and we want arbitrarily small compact PL dominations of the pair of total spaces, with bounded dimension. Let $p: E \rightarrow B$ denote the fibration, and choose a triangulation of the base. We construct dominations $j: E \longrightarrow K, r: K \longrightarrow E$ and $h: r j \sim 1$ so that if $C$ is a subcomplex of the triangulation then $(r p)^{-1} C \rightarrow E \mid C$ gives a domination. The radius of the homotopy is then bounded by the size of the triangulation, so arbitrarily small dominations are obtained by beginning with very fine triangulations. Also these can be constructed by induction on skeleta in the triangulation, so the problem reduces to extending over $E \mid \Delta^{k}$ a domination of $E \mid \partial \Delta^{k}$.

Suppose $E\left|\partial \Delta^{k} \rightarrow K \rightarrow E\right| \partial \Delta^{k}$ and $E_{0} \rightarrow L \rightarrow E_{0}$ are dominations, where $E_{0}$ is the fiber of $E$ over the barycenter of $\Delta^{k}$. There is a homotopy trivialization $f: E_{0} \times \Delta^{k} \rightarrow E \mid \Delta^{k}$. Define $\widehat{K}$ to be the homotopy pushout of $L \leftarrow L \times \partial \Delta^{k} \rightarrow K$, where the second map is a PL map homotopic to the composition $L \times \partial \Delta^{k} \rightarrow E_{0} \times \partial \Delta^{k} \rightarrow E \mid \partial \Delta^{k} \rightarrow K$. It is straightforward to use the given data to construct a domination $E\left|\Delta^{k} \rightarrow \widehat{K} \rightarrow E\right| \Delta^{k}$ extending the one given on $K$, so this completes the construction. 
Note $\widehat{K}$ is a polyhedron of dimension $k+\operatorname{dim} L$, so if a fixed domination for the fiber is used the resulting domination of $E$ will have dimension $\operatorname{dim}(X-N)+\operatorname{dim} L$. This is bounded independently of $\varepsilon$, as required.

The next result characterizes compact layered sets up to stratified homotopy and extends Theorem 1.11 .

4.7. Theorem. Suppose $Y$ is a homotopically stratified set, $Z \subset Y$ is pure and a compact layered set.

(1) There is a compact layered $X$ and a stratified homotopy retraction $X \rightarrow$ $Y$ which is a homeomorphism on $Z$, if and only if strata, and fibers of holinks of strata, of $Y$ are dominated by finite complexes.

(2) If $Y$ is stratified dominated as in (1), then there is $X \rightarrow Y$ as in (1) which is a stratified homotopy equivalence, if and only if for each stratum component $A$ the Wall finiteness obstruction $\sigma(A)$ is taken to 0 by

$$
\tilde{K}_{0}\left(\mathbf{Z} \pi_{1} A\right) \rightarrow H_{0}(\text { cone } \delta A, \delta A ; \mathscr{S}(q)) .
$$

Before proving this we give a corollary which illustrates its use.

Corollary; Layered pullbacks. Suppose $Y \subset X$ is a pure subset of a compact layered set and $f: Z \rightarrow Y$ is a stratified homotopy equivalence with $Z$ compact layered. Then there is a stratified homotopy equivalence $F: W \rightarrow Y$ with $W$ compact layered so that $F^{-1}(Y)=Z, F \mid Z=f$, and $W-Z$ has $P L$ strata.

Note that by letting $Y$ be empty we see that a compact layered set is stratified homotopy equivalent to a PL one. This extends (for compact layered sets) the Replacement Lemma 4.3, which changes only the top stratum.

Proof of the Corollary. First construct an extension $W$ of $Z$ without the PL conclusion. Form some extension as a homotopically stratified set, $\widehat{F}: \widehat{W} \rightarrow X$, using the pullback construction of 3.7. The strata and fibers of holinks have the same homotopy type as those of $X$, so are dominated by finite complexes. According to the theorem there is a compact layered extension with the same stratified homotopy type if, for each stratum $B$ of $\widehat{W}$, the image of $\sigma(B)$ in $H_{0}$ (cone $\delta B, \delta B ; \mathscr{S}(q)$ ) is trivial. Let $A$ denote the image stratum of $X$. The induced homomorphism $\widehat{F}_{*}: H_{0}(\operatorname{cone} \delta B, \delta B) \rightarrow H_{0}(\operatorname{cone} \delta A, \delta A)$ takes the image of $\sigma(B)$ to the image of $\sigma(A)$, which vanishes since $X$ is compact layered. $\widehat{F}_{*}$ is an isomorphism since $F$ is a stratified homotopy equivalence, so the image of $\sigma(B)$ is also trivial. Therefore according to the theorem $\widehat{W}$ can be replaced by a compact layered set.

To obtain the PL conclusion suppose as an induction hypothesis that $W_{n} \rightarrow$ $X$ is a stratified homotopy equivalence with $W_{n}$ a compact layered extension of $Z$, and strata in $W_{n}^{n}-Z$ are PL. Apply the Replacement Lemma 4.3 to $W_{n}^{n+1} \cup Z$ to change the $(n+1)$-strata not in $Z$ to be PL. This gives a layered 
set stratified equivalent to $W_{n}^{n+1} \cup Z$. Apply the non-PL version to extend this to $W_{n+1} \rightarrow W_{n}$. This reproduces the induction hypothesis, so by induction we get the desired $W$.

Proof of 4.7. To prove the "only if" part of both (1) and (2) we show that if $X$ is a compact layered set then strata, and fibers of holinks of strata, are dominated by finite complexes, and the finiteness obstructions of strata satisfy the given condition.

Let $A$ be a component of the $n$-stratum of $X$. Since the statement does not involve higher strata we assume $X=X^{n}$. Apply the Replacement Lemma 4.3 to $X$ to obtain an equivalent compact layered set $\widehat{X}$ whose top stratum is PL. This does not change the homotopy type of strata or holinks, so it is sufficient to show that $n$-strata of $\widehat{X}$ satisfy the conditions.

$\left(\hat{X}, X^{n-1}\right)$ satisfies the condition of $4.6(2)$, so by (3) the fibers of the holink are finitely dominated.

Since $X^{n-1}$ is reverse tame in $\widehat{X}$ there is a homotopy from the identity of $\widehat{X}-X^{n-1}$ to a map into $\widehat{X}-U$, where $U$ is a neighborhood of $X^{n-1}$. This gives a domination of $\widehat{X}-X^{n-1}$. Since there are arbitrarily small neighborhoods of $X^{n-1}$ with compact PL complement, we may assume $U$ has this property. This gives a domination of the stratum by a finite complex.

We refine this construction to get information about the finiteness obstruction. Let $\hat{A}$ be the stratum component corresponding to $A$. The definition of reverse tameness is that given a neighborhood $U$ of $\delta A$ which retracts to $\delta A$, and $\varepsilon>0$, there is a neighborhood $V$ (with compact PL complement) and a homotopy of $\hat{A}$ rel $\widehat{A}-U$ into $\hat{A}-V$, which has radius less than $\varepsilon$ when retracted into $\delta A$. This defines a domination of $\widehat{A}$ by $\widehat{A}-V$. Since the homotopy is the identity on $\hat{A}-U$, it also defines a domination of $U$ by $U-V$. If we choose $U$ closed and PL then this is a domination by a finite complex. Since $U$ and $\widehat{A}$ differ by a finite complex, the finiteness obstruction of $\hat{A}$ is the image of the obstruction of $U$ under $\widetilde{K}_{0}\left(\mathbf{Z} \pi_{1} U\right) \rightarrow \widetilde{K}_{0}\left(\mathbf{Z} \pi_{1} A\right)$.

The domination of $U$ has radius $\varepsilon$ over $\delta A$. Also the retraction to $\delta A$ factors through $p$ : holink $(\widehat{A} \cup \delta A, \delta A) \rightarrow \delta A$. Therefore according to Theorem 1.3 of [13 I] if $\varepsilon$ is small enough there is a controlled finiteness obstruction $q_{0}(U)$ defined in $H_{0}(\delta A ; \mathscr{S}(p))$, and the uncontrolled obstruction is the image of this in $\widetilde{K}_{0}$. This implies $\sigma(\widehat{A})$ is the image of $q_{0}(U)$ under the left homomorphism

$$
H_{0}(\delta A ; \mathscr{S}(p)) \rightarrow \widetilde{K}_{0}\left(\mathbf{Z} \pi_{1} A\right) \rightarrow H_{0}(\operatorname{cone} \delta A, \delta A ; \mathscr{S}(p)) .
$$

This is a fragment of the exact sequence of the pair (cone $\delta A, \delta A$ ) (see the discussion after 1.8) so is exact. Therefore the image of $\sigma(A)$ in the right group is trivial, as required for (2).

The "if" direction of both parts proceeds by induction on strata, so suppose $Y$ satisfies the hypotheses, $X_{n}$ is compact layered, and $f: X_{n} \rightarrow Y^{n} \cup Z$ is a domination, for (1), or a stratified homotopy equivalence, for (2). The objective 
is to construct an appropriate $X_{n+1} \rightarrow Y^{n+1} \cup Z$ by adding $(n+1)$-strata to $X_{n}$.

The first step is to extend $X_{n}$ to a homotopically stratified set as in 3.7. Pull holink $\left(Y^{n+1} \cup Z, Y^{n} \cup Z\right) \rightarrow\left(Y^{n} \cup Z\right)$ back to $X_{n}$, and denote the result by $p: E \rightarrow X_{n}$. Then define $\widehat{X}_{n+1}$ to be the homotopy pushout of $X_{n} \leftarrow$ $E \rightarrow\left(Y^{n+1}-Y^{n} \cup Z\right)$. According to $3.7, \widehat{X}_{n+1} \rightarrow Y^{n+1} \cup Z$ is a stratified homotopy equivalence. Write it more elaborately as the homotopy pushout of $\operatorname{cyl}(p) \leftarrow E \rightarrow\left(Y^{n+1}-Y^{n} \cup Z\right)$, then we construct PL approximations to this by approximating the pieces.

$p: E \rightarrow X_{n}$ is a stratified system of fibrations satisfying 4.6(3), so by $4.6(2)$ it is fiber homotopy equivalent to the homotopy link of a compact PL extension of $X_{n}$. More precisely, the proof shows that there is a compact $W \supset X_{n}$ which is strictly rel $X_{n}$ homotopy equivalent to the mapping cylinder $\operatorname{cyl}(p)$, and $W-X_{n}$ is PL. Choose a finte PL domination $K \rightarrow E$ (using 4.6(1)), and another $L \rightarrow\left(Y^{n+1}-Y^{n} \cup Z\right)$. Then a domination of $\widehat{X}_{n+1}$ is obtained by taking the homotopy pushout of the dominations, $W \leftarrow K \rightarrow L$. This completes the proof of $4.7(1)$.

To complete (2) we suppose $X_{n} \rightarrow Y^{n} \cup Z$ is a stratified homotopy equivalence and determine when the domination can be replaced by a stratified homotopy equivalence. Denote the pushout of dominations constructed above by $J$ and change the definition of $\widehat{X}_{n+1}$ by substituting $W$ in place of $\operatorname{cyl}(p)$. Then the domination $r: J \rightarrow \widehat{X}_{n+1}$ is a homeomorphism on a neighborhood of $X_{n}$. Choose a closed such neighborhood $N$ so that $N-X_{n}$ is PL. Let $M$ denote the closure of the complement and $P$ the intersection, so $\widehat{X}_{n+1}=M \cup_{P} N$. Then $r^{-1}(M) \rightarrow M$ is a domination which is a homeomorphism on $P$.

If there is a finite complex pair homotopy equivalent to $\left(r^{-1}(M), P\right)$ then we can take the union with $N$ to obtain the desired compact layered set equivalent to $Y^{n} \cup Z$. $M$ may be enlarged a little so that for each component $A$ of $Y^{n+1}-Y^{n} \cup Z$ the inclusion $M \cap A \rightarrow A$ is 1 -connected. In this case the obstruction to finding such finite pairs are the Wall obstructions $\sigma(M \cap A) \in$ $\widetilde{K}_{0}\left(\mathbf{Z} \pi_{1}(A)\right)$.

The next step is to show that $\sigma(M \cap A)+q_{0}\left(\operatorname{end}(N \cap A)=\sigma(A)\right.$ in $\widetilde{K}_{0}\left(\mathbf{Z} \pi_{1}(A)\right)$. To see this use the reverse tameness to pull $N$ outside a neighborhood $U$ of $X_{n}$ with compact PL complement in $N$. This gives a domination of $A$ by the finite complex $r^{-1}(A-U)$, so this domination has invariant $\sigma(A)$. However it is still a homeomorphism on $P$, so can be split into two pieces. One is $\sigma(M)$ as above, and the other is the domination of $N \cap A$ by $(N-U) \cap A$. The analysis of part (1) shows the second piece to be the image of the end invariant $q_{0}(N \cap A)$. Since disjoint unions of dominations gives the sum of obstructions, the desired formula follows. 
Now recall that the hypothesis in (2) is that $\sigma(A)$ has trivial image in $H_{0}$ (cone $\left.\delta A, \delta A ; \mathscr{S}(q)\right)$. Going back one term in the exact sequence, this means it is in the image of $H_{0}(\delta A ; \mathscr{S}(q))$. Since $q_{0}(N \cap A)$ is also in this image, the difference $\sigma(M \cap A)$ must also be in this image. Suppose it is the image of an element $\alpha$.

Regard $W \cap A$ as a proper polyhedron over $\delta A \times(0,1]$ by the composition $W \rightarrow \operatorname{cyl}(p) \rightarrow X_{n} \times[0,1]$. Use the realization clause of [13 II, 1.6] to find a proper polyhedron $T$ and $T \rightarrow N \cap A$ which is a controlled equivalence over a neighborhood of $N$, with $q_{1}=\alpha$. (The referenced result is actually an $h$-cobordism theorem. Thicken $N \cap A$ to a manifold, then $T$ is obtained as an $h$-cobordism of that manifold.) According to [13 II, 1.8(b)] the end obstruction of $T$ over $\delta A$ is the difference $q_{0}(\operatorname{end}(N))-\partial \alpha$, where $\partial$ is the isomorphism $\partial: H_{1}^{l f}(\delta A \times(0,1) ; \mathscr{S}(p)) \simeq H_{0}(\delta A ; \mathscr{S}(p))$.

Repeat the construction of the domination using $T$ as a neighborhood of $X_{n}$ instead of $W$. The interior piece corresponding to $M$ now has invariant $\sigma(A)-q_{0}(\operatorname{end}(T))$, which has been arranged to vanish. We conclude that this piece can be replaced by a finite complex, giving the required compact layered set equivalent to $Y^{n+1} \cup Z$.

4.8. Simple homotopy. We begin the proof of the simple homotopy Theorem 1.10 with the construction of the invariant $\tau$, and the proof of the additivity property.

Suppose $f: X \rightarrow Y$ is a stratum-preserving map of compact layered sets which is a homotopy equivalence on each stratum. Choose a stratum component $A$ of $Y$, with corresponding component $B$ in $X$. Then we define the corresponding component of $\tau(f), \tau_{A} \in H_{1}(\operatorname{cone} \delta A, \delta A ; \mathscr{S}(q))$.

Replace $X$ and $Y$ by the closures $\bar{B}, \bar{A}$, so the strata of interest are the top strata. Then use the Replacement Lemma 4.3 to replace them by finite dimensional PL strata.

The invariant $\tau_{A}$ is defined by refining the geometric interpretation of the exact sequence following 1.8. The exact sequence of homology groups

$$
\begin{aligned}
\cdots \rightarrow H_{1}(\delta A ; \mathscr{S}(p)) & \rightarrow \mathrm{Wh}\left(\pi_{1} A\right) \rightarrow H_{1}(\text { cone } \delta A, \delta A ; \mathscr{S}(q)) \\
& \rightarrow H_{0}(\delta A ; \mathscr{S}(p)) \rightarrow \widetilde{K}_{0}\left(\mathbf{Z} \pi_{1} A\right) \rightarrow \cdots
\end{aligned}
$$

comes from $\pi_{0}$ applied to a homotopy fibration of homology spaces [13 II, §8],

$$
\mathbf{H}_{1}(\operatorname{cone} \delta A, \delta A ; \mathscr{S}(q)) \rightarrow \mathbf{H}_{0}(\delta A ; \mathscr{S}(p)) \rightarrow \mathbf{H}_{0}(\operatorname{cone} \delta A ; \mathscr{S}(p)) \text {. }
$$

Therefore to define an element in $H_{1}=\pi_{0} \mathbf{H}_{1}$, it is sufficient to construct a point in $\mathbf{H}_{0}(\delta A ; \mathscr{S}(p))$ and a nullhomotopy of its image in $\mathbf{H}_{0}(\operatorname{cone} \delta A ; \mathscr{S}(p))$.

$\delta A$ is tame and reverse tame in $\bar{A}$ since $Y$ is a layered set. If $\varepsilon>0$ we can choose a closed neighborhood $N$ which nearly strictly $\varepsilon$ deformation retracts in $\bar{A}$ to $\delta A$. This gives an $\varepsilon$ factorization of $N-\delta A$ through $p:$ holink $(\bar{A}, \delta A) \rightarrow \delta A$. Next there is a smaller open neighborhood $U$ so that 
there is an $\varepsilon$ homotopy of $A$ into $A-U$, so that the nonconstant part of the homotopy maps into $N-\delta A$. This gives an $\varepsilon$ domination of $N-\delta A$ by $N-U$, measured in $\delta A$. Since $A$ is PL, $U$ and $N$ can be chosen so that $N-U$ is a compact polyhedron. According to [13 II, 1.3] (and its proof in $\S \S 6.3,6.4)$ if $\varepsilon$ is small enough this determines a point in $\mathbf{H}_{0}(\delta A ; \mathscr{S}(p))$, whose homotopy class in $\pi_{0}$ is denoted $q_{0}($ end $A)$.

Similarly there is a domination of the end of $B$, which determines a point $q_{0}($ end $B)$ in $\mathbf{H}_{0}(\delta B ; \mathscr{S}(p))$. The map $f$ induces a map of homology spaces, so there is an image point $f_{*} q_{0}$ (end $B$ ) in the homology space for $A$. The difference $q_{0}($ end $A)-f_{*} q_{0}($ end $B)$ is the starting point for the definition of $\tau_{A}$. The definition will be completed by construction of a nullhomotopy of the image of this difference, in $\mathbf{H}_{0}(\operatorname{cone} \delta A ; \mathscr{S}(p))$.

The invariant $q_{0}$ (end $A$ ) corresponds to the domination of $N-\delta A$ by $N-U$. Over the cone we can replace the complement of $N$ to get a domination of $A$ by $A-U$. Also contract the control map to the cone point to get control space a point. This defines a concordance of dominations, and so a homotopy of the image of $q_{0}($ end $A)$. Similarly the image of $q_{0}(\operatorname{end} B)$ is homotopic to the invariant of the uncontrolled domination of $B$ by $B-V$, some appropriate $V$.

The difference of these homotopies gives a homotopy of $q_{0}$ (end $A$ ) $f_{*} q_{0}($ end $B)$ to the difference of dominations $A-U \rightarrow A$ and $B-V \rightarrow B$. But we have assumed $B \rightarrow A$ is a homotopy equivalence, so the proof of the homotopy invariance of finiteness obstructions gives a homotopy to the difference of two dominations of $A$. Finally the proof that the finiteness obstruction is independent of the choice of domination gives a homotopy of this difference to the basepoint.

This completes the construction of $\tau$, and we now consider the additivity.

Suppose $f: X \rightarrow Y$ and $g: Z \rightarrow X$ are as specified in 1.10(2). Let $A$ be a stratum component in $Y$ with corresponding components $B$ in $X$ and $C$ in $Z . \tau_{A}(f)+f_{*} \tau_{B}(g)$ begins with $q_{0}($ end $A)-f_{*} q_{0}($ end $B)+f_{*}\left(q_{0}(\right.$ end $B)-$ $g_{*} q_{0}$ (end $\left.C\right)$ ), which by cancelling the inverses is canonically homotopic to the starting point of $\tau_{A}(f g)$. Similarly the paths to differences of dominations of $A, B, C$, are homotopic by cancelling $B$ terms.

We now have the sum of two paths, one from the difference of dominations of $A$ and $B$ to the basepoint using the fact that $f$ is a homotopy equivalence, and a similar one for $B$ and $C$. We want a homotopy from the sum of these paths, to the path from the difference of $A$ and $C$, essentially cancelling the two occurrences of $B$. The construction of such a homotopy comes from collapsibility properties of mapping cylinders, essentially as in the proof of the additivity of Whitehead torsion [13 II, §6.6].

The next lemma relates $\tau$ to controlled simple homotopy in a special case. The setting is [13 II, Theorem 1.4], which asserts that if $\varepsilon$ : open cone $\delta A \rightarrow$ $(0, \infty)$ is small enough then an $\varepsilon$ homotopy equivalence of proper polyhedra has an associated invariant $q_{1}$ in the locally finite homology of the open cone. 
4.9. Lemma. Suppose $A$ is a stratum component of a compact layered set $Y$, $X$ is compact layered, and $f: X \rightarrow Y$ is a homeomorphism on $\delta A$, and a strict rel $\delta A$ homotopy equivalence. Then $f^{-1}(A) \rightarrow A$ is a controlled homotopy equivalence over the open cone of $\delta A$ so $q_{1}(f)$ is defined, and $\tau_{A}$ is the image of this under the natural isomorphism

$$
H_{1}(\operatorname{cone} \delta A, \delta A ; \mathscr{S}(q)) \stackrel{\simeq}{\longrightarrow} H_{1}^{l f}(\text { open cone } \delta A ; \mathscr{S}(q)) \text {. }
$$

Proof. By $2.4(2), \bar{A}$ is strictly rel $\delta A$ homotopy equivalent to the homotopy pushout of $\delta A \leftarrow \operatorname{holink}(\bar{A}, \delta A) \rightarrow$. Compose this with the projection of the pushout to the cone on $\delta A$ to get a strict map $j: \bar{A} \rightarrow$ cone $\delta A$. Then since $f$ is a strict rel $\delta A$ homotopy equivalence, the Control Lemma 4.4 applies to give radial homeomorphism $r$ of the cone so that with respect to the reference map $r j, f: B \rightarrow A$ is an $\varepsilon$ homotopy equivalence. This is what is meant by $f$ being "controlled" and implies $q_{1}(f)$ is defined.

According to [13 II, 1.8b], $\partial q_{1}(f)=q_{0}(A)-q_{0}(B)$. Therefore $q_{1}$ and $\tau$ are both determined by homotopies of this difference to the basepoint in $\mathbf{H}_{0}(\operatorname{cone} \delta A ; \mathscr{S}(p))$. A minor extension of the proof in [13 II, §6.6] gives a homotopy between these homotopies.

4.10. Proof of 1.10. Parts (1) and (3) are proved in 4.8, so here we complete (2) and (4).

To prove (2) suppose $A$ is a component of the $n$-stratum of $Y$ and $\alpha \in$ $H_{1}$ (cone $\delta A, \delta A ; \mathscr{S}(q)$ ). It is sufficient to show there is a compact layered set and stratified homotopy equivalence $f: X \rightarrow Y$ with $\tau_{A}(f)=\alpha$ and $\tau_{B}(f)=$ 0 if $B$ is a stratum component in $Y^{n}-A$. To get the general statement proceed by induction on skeleta, assuming $X_{n-1} \rightarrow Y$ has the desired invariants on the $(n-1)$-skeleton. Then use the single-stratum case and the additivity of (3) to construct $X_{n} \rightarrow X_{n-1}$ so that the composition $X_{n} \rightarrow Y$ has the desired invariants on the $n$-skeleton.

To prove the single-stratum case first use the Replacement Lemma 4.3 to obtain a compact layered set equivalent to $Y^{n}$ with manifold $n$-stratum. Apply the $h$-cobordism realization theorem [13 II, $1.2 \mathrm{~b}]$ to find an $h$-cobordism of the stratum over $A$ with controlled torsion $q_{1}=-\alpha$. Let $X_{n}^{n}$ denote the layered set with $(n-1)$-skeleton $Y^{n-1}$ and $n$-skeleton the $h$-cobordism. The retraction of this $h$-cobordism to the end gives $X_{n}^{n} \rightarrow Y^{n}$ which is a strict rel $Y^{n-1}$ homotopy equivalence. In this case Lemma 4.9 identifies the controlled obstruction with $\tau_{A}$, so this map has $\tau_{A}=\alpha$. Extend $X_{n}^{n} \rightarrow Y$ to some stratified homotopy equivalence of layered sets $X_{n} \rightarrow Y$ using the corollary to 4.7. This has the properties needed for the "single-stratum" statement above, so completes the proof of $1.10(1)$. 
For the "if" direction of (4) we show that a cell-like $f: X \rightarrow Y$ has $\tau(f)=0$. Regard $Y$ as obtained from $X$ by identifying point inverses to points, then intermediate spaces $X \rightarrow X_{n} \rightarrow Y$ are defined by making the identifications only in the $n$-skeleton. These are compact layered sets. $X \rightarrow X_{n-1}$ is a homeomorphism on the $n$-stratum so $\tau$ is trivial there. By additivity, then, $X_{n-1} \rightarrow Y$ has the same $n$-skeleton invariants as $f$.

$X_{n-1} \rightarrow Y$ is cell-like and a homeomorphism on the $(n-1)$-skeleton. Therefore if $A$ is a component of the $n$-skeleton, the inverse image of the closure $\bar{A}$ is strictly rel $\delta A$ homotopy equivalent to $\bar{A}$. Under these conditions Lemma 4.9 identifies $\tau_{A}$ with the controlled torsion of the map of strata. Ideally we would now refer to [13 II, Theorem 1.4], which states that cell-like maps have trivial controlled torsion. Unfortunately this theorem is only stated for PL maps, so some additional argument is required.

The reference map used for the controlled torsion in 4.9 is the projection from the open mapping cylinder of $g: \operatorname{holink}(\bar{A}, \delta A) \rightarrow A$ to the open mapping cylinder of $\delta A \rightarrow \mathrm{pt}$ (the open cone). The inverse of the strict homotopy equivalence of 2.4 gives a commutative diagram

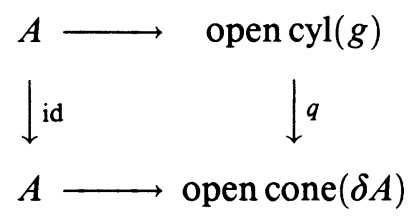

Since the map from the inverse image of $A$ to $A$ is cell-like it is a controlled homotopy equivalence with control measured in $A$ itself. This means there is a controlled torsion defined in $H_{1}^{l f}(A ; \mathscr{S}$ (id)), and the invariant of 4.9 is the image of this under the homomorphism $H_{1}^{l f}(A ; \mathscr{S}($ id $)) \rightarrow H_{1}^{l f}(A ; \mathscr{S}(q))$ induced by the commutative diagram. But $\mathscr{S}$ (id) has fiber the finite structure spectrum associated to a point, which is contractible. Therefore the group is trivial, and the invariant vanishes.

Finally we consider the "only if" direction of 1.10(4); a stratified homotopy equivalence with $\tau=0$ is obtained from cell-like maps. This proceeds by induction on strata, so we suppose it is true for $(n-1)$-skeleta and show it is true for $n$-skeleta.

Suppose $Y^{n}=Y$ and $f: X \rightarrow Y$ is a stratified homotopy equivalence. Apply the induction hypothesis to obtain $Z^{n-1}$, cell-like maps $g^{n-1}: Z^{n-1} \rightarrow X^{n-1}$ and $h^{n-1}: Z^{n-1} \rightarrow Y^{n-1}$, and a stratified homotopy $f^{n-1} g^{n-1} \sim h^{n-1}$. According to the layered pullback lemma (corollary to 4.7) there is an extension of $Z^{n-1}$ and $g^{n-1}$ to a stratified homotopy equivalence $\hat{g}: \hat{Z} \rightarrow X$. There is also an extension of $\hat{h}$ : According to Proposition 2.2 it is sufficient to find a morphism of the holink diagram. holink $\left(\widehat{Z}, Z^{n-1}\right) \rightarrow Z^{n-1}$ is the pullback of holink $\left(X, X^{n-1}\right) \rightarrow X^{n-1}$. These are stratified systems of fibrations, so the stratified homotopy $f^{n-1} g^{n-1} \sim h^{n-1}$ induces, by 3.3 and the 
lemma following 2.7, a fiber homotopy equivalence of this with the pullback of $\operatorname{holink}\left(Y, Y^{n-1}\right) \rightarrow Y^{n-1}$. This homotopy commutes with the homotopy equivalences of the $n$-strata, so gives a morphism of the diagram. Similarly there is an extension of the homotopy to a stratified homotopy $f \hat{g} \sim \hat{h}$, using 2.3 .

We may assume that $\tau(\hat{g})=\tau(\hat{h})=0 . \tau(\hat{g})$ can only be nonzero on the $n$-stratum because the map is cell-like on lower skeleta. If the top invariant is nonzero, compose with a map (from the realization assertion 1.10(2)) which is the identity on the $(n-1)$-skeleton and has the negative invariant. The composition with $\hat{g}$ then has trivial invariant by the composition formula. Since $\tau(f)=0$, this formula also implies the composition with $\hat{h}$ is trivial.

The proof is now reduced to the case where $X^{n-1} \rightarrow Y^{n-1}$ is cell-like. This case can be applied to the $\hat{Z} \rightarrow X$ constructed above to get $\widehat{Z} \leftarrow \widetilde{Z} \rightarrow X$, and then applied to the composition $\tilde{Z} \rightarrow \widehat{Z} \rightarrow Y$ to obtain a layered set $Z$ satisfying the conclusion of the induction step.

If $X^{n-1} \rightarrow Y^{n-1}$ is cell-like we can construct an intermediate space $X \rightarrow$ $W \rightarrow Y$ as in the "if" part of the proof above, with the same $n$-stratum as $X$ and same $(n-1)$-skeleton as $Y . W \rightarrow Y$ is a strict rel $Y^{n-1}$ homotopy equivalence, so according to the control lemma is a controlled homotopy equivalence over the open cones of frontiers of stratum components.

Let $\hat{Y}$ denote the layered set obtained by replacing the top strata by a manifold mapping cylinder as in 4.3. Arrange also that the dimension of this manifold is at least $2 \operatorname{dim}\left(X^{n}-X^{n-1}\right)+2$. In this case $W \rightarrow \widehat{Y}$ can be approximated rel $Y^{n-1}$ to be a 1-LC embedding on top strata; $\left(X^{n}-X^{n-1}\right) \subset\left(\widehat{Y}^{n}-\widehat{Y}^{n-1}\right)$. Let $V$ denote a mapping cylinder neighborhood.

Since the inclusion is a controlled homotopy equivalence over the open cones, the difference $\widehat{Y}^{n}-\widehat{Y}^{n-1}-$ int $V$ is a controlled $h$-cobordism. The controlled torsion is the same as that of the inclusion, which is trivial since by 4.9 it is the same as the $n$-stratum part of the stratified torsion of $f$. By [13 II, 1.6] this implies the $h$-cobordism has a controlled product structure. This gives a strict rel $Y^{n-1}$ homotopy to a homeomorphism $W \simeq\left(V \cup Y^{n-1}\right)$.

Define $Z=X^{n-1} \cup V$, then the retraction in the mapping cylinder gives a cell-like $Z \rightarrow X$. The composition $Z \rightarrow\left(Y^{n-1} \cup V\right) \simeq W \rightarrow \widehat{Y} \rightarrow Y$ gives a cell-like map to $Y$. These give appropriate extensions of the maps given on the $(n-1)$-skeleta, so complete the proof of the theorem.

\section{MANifOld HOMOtopiCALly STRATIFIED SETS}

These are defined and discussed in 5.1. The Isotopy Extension Theorem 1.1 and the Boundary Collaring Theorem 1.2 are proved in 5.2 and 5.3 respectively. The group action results, 1.4 and 1.5 , are proved in 5.4. The discussion of $h$-cobordisms in 5.5 begins with the proof that manifold homotopically stratified sets are layered, so the material of the latter part of $\S 4$ applies. Then 
$h$-cobordisms are shown to be characterized by their torsions in 5.6, extending Theorem 1.8.

5.1. Definition. A manifold homotopically stratified set is a homotopically stratified locally compact metric space whose strata are manifolds, which satisfy a boundary condition. Define the boundary to be the union of the boundaries of the strata; $\partial X=\bigcup_{i} \partial\left(X^{i}-X^{i-1}\right)$. Then $\partial X$ is required to be closed and homotopically transverse to the skeleta of $X$. (Homotopy transversality is defined in 3.4.)

The homotopy transverse condition on the boundary is, by 3.4 , equivalent to $\partial X$ being a stratum-preserving neighborhood deformation retract in $X$. It is also equivalent to the mapping cylinder of the inclusion being a homotopically stratified set with respect to the given filtration. If $X$ is a manifold homotopically stratified set with boundary we define the set obtained by absorbing the boundary into the filtration to be the same space with filtration whose $i$-skeleton is $X^{i} \cup \partial X^{i+1}$. This has strata manifolds without boundary, but the original $\partial X$ is not lost; it is a pure subset and can be characterized by certain homotopy link conditions. Boundaries are included in the definition because they considerably simplify notation, for example in the $h$-cobordism theorem.

5.2. Proof of 1.1. The main ingredient is the Isotopy Extension Theorem 2.1 of [13 IV]. That paper was written before the development of the tameness and homotopy link material, so the result is stated in terms of the more awkward "eventual equivalence of neighborhoods." These are translated as follows:

(1) $Y \subset X$ has "neighborhoods eventually equivalent to neighborhoods in a mapping cylinder" if and only if it is tame (and then the map is $\operatorname{holink}(X, Y) \rightarrow Y)$.

(2) If $A \supset Y$ and $B \supset Y$, and $Y$ is tame in both, then $Y$ "has eventually equivalent neighborhoods" in these spaces if and only if there is a fiber homotopy equivalence $\operatorname{holink}(A, Y) \rightarrow \operatorname{holink}(B, Y)$ over $Y$.

Now suppose $(V, W)$ and $(X, Y)$ are pairs and

$$
F_{0} \cup f:(V \times\{0\} \cup W \times I, W \times I) \rightarrow(X, Y)
$$

is strict. Then [13 IV, 2.1] translates to:

Theorem. Suppose $V, X$ are locally compact metric, $X-Y$ is a manifold of dimension at least 5, $Y$ is tame and a finite dimensional $A N R, \operatorname{holink}(X, Y) \rightarrow Y$ is approximately 2-equivalent to a stratified system of fibrations, and $F_{0}:(V-W) \rightarrow(X-Y)$ is a homeomorphism. Then there is a strict homotopy extending $F_{0} \cup f$ and which is an isotopy of complements, if and only if there is a fiber homotopy equivalence holink $(X \times I, Y \times I) \rightarrow \operatorname{holink}\left(Y \times I \cup_{f} V \times I, Y \times I\right)$ over $Y \times I$ extending the one over $Y \times\{0\}$ induced by $F_{0}$.

Here $Y \times I \cup_{f} V \times I$ is the union with points in $W \times I$ identified to their $f$ images in $Y \times I . \quad X-Y$ should not have boundary (i.e. the boundary is 
contained in $Y$ ). Note that 2.3 determines when there is a strict homotopy which is not an isotopy.

This statement simplifies considerably in the context of isotopies beginning with the identity. If $f$ is an isotopy then $\operatorname{holink}(Y \times I \cup V \times I, Y \times I)$ is the pullback of holink $(Y \cup X \times I, Y \times I)$, which is a product. The fiber equivalence of holinks is therefore equivalent to a covering of $f$ by a fiber homotopy from $f_{0}^{*} \operatorname{holink}(X, Y)$ to $\operatorname{holink}(X, Y)$. Now suppose $Y$ is a finite dimensional locally compact ANR which is tame in $X, X-Y$ is a manifold, $\partial X \subset X$ is closed and intersects $X-Y$ in its boundary, and holink $(X, Y)$ is a stratified system of fibrations with $Y \cap \partial X$ pure in the filtration.

Corollary. Suppose $X, Y$ satisfy the conditions above, $X-Y$ has dimension at least 5, and $f$ is an isotopy of $Y$ fixing $Y \cap \partial X$. Then it extends to an isotopy of $X$ fixed on $\partial X$ if and only if it is covered by a fiber homotopy of holink $(X, Y)$ beginning with the identity and constant over $Y \cap \partial X$.

Theorem 1.1 follows from this by induction on skeleta, using the fact that a stratum-preserving isotopy is covered by a fiber homotopy of holinks (3.2, 3.3).

5.3. Proof of 1.2. Again we proceed by induction on skeleta. For the induction step consider closed subsets $Y \times[0, \infty) \subset X \supset \partial X$, with $\partial X \cap Y \times[0, \infty)=$ $Y \times\{0\}$, and $X-Y$ a manifold which intersects $\partial X$ in its boundary.

Lemma. Suppose in addition $X$ is locally compact, $Y \times[0, \infty)$ is tame, $Y$ is a finite dimensional $A N R$, $\operatorname{holink}(X, Y \times[0, \infty))$ is fiber homotopy equivalent to holink $(\partial X, Y \times\{0\}) \times[0, \infty)$, and holink $(\partial X, Y \times\{0\})$ is a stratified system of fibrations. If $\operatorname{dim}(X-Y) \geq 5$, or $=4$ and the fibers of holink $(\partial X, Y \times\{0\})$ have poly-(finite or cyclic) fundamental groups, then the open collar $Y \times[0, \infty)$ extends to an open collar of $\partial X$.

We will show there is a neighborhood $N$ of $Y \times\{0\}$ in $\partial X$ and an extension of the collar to a collar of $N$ in $X$. The standard collaring theorem for manifolds then implies it extends to a collar of $\partial X$. The collar of $N$ is obtained by constructing a controlled $h$-cobordism from a neighborhood of $N$ to a collar. The obstruction group for this $h$-cobordism is trivial, so the ends are homeomorphic and a collar exists. In the next statement, $Y$ is a locally compact ANR and $p: E \rightarrow Y$ is a stratified system of fibrations.

Proposition. Suppose $\varepsilon: Y \times[0, \infty) \times(0, \infty) \rightarrow(0, \infty)$ and $n$ are given. Then there is $\delta: Y \times[0, \infty) \times(0, \infty) \rightarrow(0, \infty)$ such that if $W^{n} \rightarrow E \times[0, \infty) \times(0, \infty)$ is $(\delta, 1)$-connected and $a(\delta, h)$-cobordism over $Y \times[0, \infty) \times(0,1)$ then a $\delta$ product structure on the boundary $h$-cobordism extends to an $\varepsilon$ product structure over $Y \times[0, \infty) \times(0,1-\varepsilon)$, provided either $n \geq 6$, or $n=5$ and fundamental groups of fibers of $p$ are poly-(finite or cyclic). 
Proof. If $\delta$ is small enough then [13 II, 1.6] associates to $W$ an invariant in

$$
H_{1}^{l f}(Y \times[0, \infty) \times(0,1) ; \mathscr{S}(p \times I \times I)),
$$

which is the trivial group. Since the invariant vanishes [13 II, 1.6] asserts the existence of the product structure if $n \geq 6$. The case $n=5$ and fundamental groups of fibers of $p$ are trivial is in [13 III]. Finally this is extended to poly(finite or cyclic) groups in [11].

Proof of the lemma. Now consider $X \times[0, \infty)$. We give a neighborhood of $Y \times$ $[0, \infty) \times[0, \infty)$ the structure of a controlled $h$-cobordism from $X \times\{0\} \rightarrow \partial X \times$ $[1, \infty)$, with the product $\partial X \times[0,1]$ as boundary $h$-cobordism. To facilitate this we change the parameterization a little. Let $W$ denote $X \times[0, \infty)$, and let $X \subset W$ denote $X \times\{0\}$. Parameterize $\partial X \times[0, \infty) \subset X \times[0, \infty)$ as $\partial X \times(\{0\} \times I \cup[0, \infty) \times\{1\}) \subset W$, and parameterize $Y \times[0, \infty) \times[0, \infty)$ as $Y \times[0, \infty) \times I \subset W$, by a homeomorphism of the second two factors which is the identity on $[0, \infty) \times\{0\} \cup\{0\} \times I$. Then $Y \times[0, \infty) \times I$ intersects $X$ in $Y \times[0, \infty) \times\{0\}$, and $\partial X \times(\{0\} \times I \cup[0, \infty) \times\{1\})$ in $Y \times(\{0\} \times I \cup[0, \infty) \times\{1\})$.

By hypothesis $Y \times[0, \infty)$ is tame in $X$, and the homotopy link is fiber homotopy equivalent to a product with respect to the $[0, \infty)$ coordinate. The first use made of this is to produce the deformation retractions needed for an $h$-cobordism. The deformations of $Y \times[0, \infty) \times I$ to $Y \times[0, \infty) \times\{i\}$, for $i=0,1$, defined by pushing in the $I$ coordinate, are covered by fiber homotopies of holink $(W, Y \times[0, \infty) \times I)$ into $\operatorname{holink}(X, Y \times[0, \infty) \times\{0\})$ and holink $(\partial X \times[0, \infty) \times\{1\}, Y \times[0, \infty) \times\{1\})$ respectively. It follows from 2.3 that the deformations extend to deformations $R_{i}$ of $W$ which are strict with respect to $Y \times[0, \infty) \times I$ and which push some neighborhood $N$ of $Y \times[0, \infty) \times I$ into $X$ and $\partial X \times[0, \infty) \times\{1\}$, when $i=0,1$ respectively. The $R_{i}$ are also the identity outside some neighborhood of $Y \times[0, \infty) \times I$, so in particular can be chosen to be proper.

The next step is to construct appropriate control map. Since $Y \times[0, \infty) \times I$ is tame in $W$ there is a nearly strict deformation retraction of a neighborhood $N$. This defines a map $N-Y \times[0, \infty) \times I \rightarrow \operatorname{holink}(W, Y \times[0, \infty) \times I)$. As above the holink is fiber homotopically a product, so let $j$ denote the composition of this map with the projection holink $(W, Y \times[0, \infty) \times I) \simeq \operatorname{holink}(\partial X, Y) \times[0, \infty) \times$ $I \rightarrow$ holink $(\partial X, Y) \times[0, \infty)$. Let $J$ denote the further composition of this with $p \times 1$ : holink $(\partial X, Y) \times[0, \infty) \rightarrow Y \times[0, \infty)$. Next choose $e: N \rightarrow[0, \infty)$ so that $e^{-1}(0)=Y \times[0, \infty) \times I$, and $(J, e)$ is proper. ( $e$ is essentially the distance from $Y \times[0, \infty) \times I$.) Then the $R_{i}$ are strict homotopies with radius 0 on $Y \times[0, \infty) \times I$, with respect to $(J, e): N \rightarrow(Y \times[0, \infty)) \times[0, \infty)$. The Control Lemma 4.4 implies that $e$ can be changed to make the $R_{i}$ have radius less than any given $\delta$, over $(Y \times[0, \infty)) \times[0,1]$.

To verify the remaining hypotheses of the Proposition, note we have assumed holink $(\partial X, Y) \rightarrow Y$ is a stratified system of fibrations. Also the map $j: N-Y \times[0, \infty) \times I \rightarrow \operatorname{holink}(\partial X, Y) \times[0, \infty)$ induces a strict local homotopy 
equivalence of $N$ with the mapping cylinder of the holink (by tameness), so again by changing $e$ we can make it $(\delta, n)$-connected over $Y \times[0, \infty) \times[0,1]$ for any given $\delta$ and all $n$, so in particular $(\delta, 1)$-connected.

Applying the Proposition, we conclude that given the hypotheses of the lemma and any $\varepsilon:(Y \times[0, \infty)) \times[0, \infty) \rightarrow[0, \infty)$ with $\varepsilon^{-1}(0)=(Y \times[0, \infty)) \times\{0\}$, we can find a function $e$ so that with respect to control function $(J, e)$, $N-Y \times[0, \infty) \times[0,1]$ is an $\varepsilon$ product over $Y \times[0, \infty) \times(0,1-\varepsilon)$. But any such product structure gives homeomorphisms of the ends which extend by the identity on $Y \times[0, \infty)$ to give a homeomorphism of a neighborhood of $Y \times[0, \infty) \times\{0\} \subset X$ to a neighborhood of $Y \times[0, \infty) \times\{1\} \subset \partial X \times[0, \infty) \times\{1\}$. This gives the required extension of the collar structure to a neighborhood.

This proof can be modified to show that in some cases $X$ itself is an open collar, generalizing a manifold result of Siebenmann.

Corollary. Suppose $\partial X$ is compact, the inclusion $\partial X \rightarrow X$ is a homotopy equivalence on each stratum, and fundamental groups of strata near the end of $X$ are the same as those of the stratum. Then a stratum-preserving homeomorphism $X^{4} \simeq\left(\partial X^{4}\right) \times[0, \infty)$ extends to a stratum-preserving homeomorphism $X \simeq(\partial X) \times[0, \infty)$.

The condition on "fundamental groups near the end" is that if $K \subset X$ is compact then there is $L \supset K$ compact so that image $\left(\pi_{1}\left(X^{k}-X^{k-1} \cup L\right) \rightarrow\right.$ $\left.\pi_{1}\left(X^{k}-X^{k-1} \cup K\right)\right)$ maps isomorphically to $\pi_{1}\left(X^{k}-X^{k-1}\right)$.

Proof. The proof proceeds by induction on skeleta, so suppose $Y \subset X$ is the next-to-top skeleton, $Y \simeq \partial Y \times[0, \infty)$, and $X-Y$ is connected. The global version of 3.5 implies that there is a stratum-preserving deformation retraction of $X$ to $\partial X$, which on $Y$ is the standard one. Let $r: X \rightarrow \partial X$ be the retraction at the end of this deformation, and let $j: X \rightarrow[0, \infty)$ be a proper map which extends the projection on $Y$. We claim that $j$ can be composed with an automorphism of $[0, \infty)$ so that $(r, j)$ is an $\varepsilon$ stratified homotopy equivalence, $\varepsilon$ measured in $[0, \infty)$. First we show how the corollary follows from the claim.

$X \times[0, \infty)$ can be parameterized, as in the proof of the lemma, to give a global $\varepsilon h$-cobordism from $X$ to $\partial X \times[0, \infty)$, with control in $[0, \infty)$. The proof of the lemma gives control in this $h$-cobordism near $Y$ over $Y \times$ $(0,1) \times[0, \infty)$. Putting these together we get a controlled $h$-cobordism over (open cone $Y) \times[0, \infty)$, from $X-Y$ to $\partial(X-Y) \times[0, \infty)$. The dimension is $\geq 6$, and the obstruction group $H_{1}^{l f}(($ open cone $Y) \times[0, \infty) ; \mathscr{S}(q))$ vanishes, so there is a product structure. In particular there is a homeomorphism $X-Y \simeq \partial(X-Y) \times[0, \infty)$. The argument in the proof of the lemma shows this extends the structure given on $Y$.

Now consider the claim that $(r, j)$ can be made an $\varepsilon$ homotopy equivalence. The concern is for the top stratum, $(X-Y) \rightarrow \partial(X-Y) \times[0, \infty) . \partial(X-Y)$ is noncompact, but by the lemma we can assume $(r, j)$ is a homeomorphism over 
a neighborhood of the end, so the unknown part is compact. The $\pi_{1}$ condition implies $(r, j)$ can be made $(\delta, 1)$ connected, so it is sufficient to show it can be made a $\delta$ homological isomorphism (with $\pi_{1}$ coefficients). This follows from reverse tamenes and reparameterization of $[0, \infty)$ as in 4.4 . We omit the details.

5.4. Proofs of 1.4 and 1.5. Suppose $G$ acts on $X$ as in 1.4. On strata the quotient is a covering space, so the strata of $X$ are manifolds if and only if those of $X / G$ are. Assuming this, they are both finite dimensional, so Proposition 3.6 applies to show that $X / G$ is homotopically stratified if and only if $X$ is. Finally, manifold homotopically stratified sets are required to be locally coct This is equivalent, in the context, to the finite index condition on isotropy subgroups. This proves 1.4.

The next result is used inductively to prove Proposition 1.5.

Proposition. Suppose $X \subset M$ is a manifold homotopically stratified set embedded as a closed and 1-LC subset in a manifold, $\partial X=X \cap \partial M$, and $\partial X$ is 1-LC in $\partial M$. Then $M$ with the filtration given by $X$ and its skeleta is a manifold homotopically stratified set.

Proof. $M$ is locally compact and the strata are manifolds, so we need to see that it is homotopically stratified. According to Proposition 4.2 this is the case if holink $\left(M-X^{i}, X^{i+1}-X^{i}\right) \rightarrow\left(X^{i+1}-X^{i}\right)$ is a fibration for all $i$. But $X^{i+1}-X^{i}$ is a proper 1-LC submanifold of $M-X^{i}$, so it is locally flat. The homotopy link of a locally flat submanifold is a spherical fibration, and in particular a fibration.

Proof of 1.5. In 1.5(1) we are given a finite collection of 1-LC submanifolds $\left\{M_{s}\right\}$ closed under intersection, and want to show $M$ filtered by the subsets $M^{i}=\bigcup\left\{M_{s}: \operatorname{dim} M_{s} \leq i\right\}$ is a manifold homotopically stratified set. It is locally compact and has manifold strata, so the problem is to show it is homotopically stratified. Assume as an induction hypothesis that the proposition is known if there are $k$ or fewer strata, and suppose that $M$ has $k+1$. Then each of the $M_{s}$ of smaller dimension than $M$ satisfy the induction hypothesis, so are homotopically stratified. The next-to-largest skeleton $M^{n-1}$ has the same components of strata as the $M_{s}$ so it is homotopically stratified. $M^{n-1}$ is 1-LC in $M$ because the closed subsets $M_{s}$ are, so the proposition applies to show $M$ is homotopically stratified.

In 1.5(2) the collection is assumed to be locally flat. Since homotopy stratification is a local property it is sufficient to show Euclidean space is homotopically stratified by a collection of linear subspaces. Let $V_{i}$ denote subspaces of $V$, and let $V_{1}$ be the minimal one. By induction on skeleta it is sufficient to show that $V_{1} \subset\left(V-\bigcup V_{i}\right) \cup V_{1}$ is tame and has fibered homotopy link. Since $V_{1} \subset V_{i}$, all $i$, there is an isomorphism $V \simeq V^{\perp} \times V_{1}$ so that each $V_{i}$ corresponds to 
$V_{i}^{\perp} \times V_{1}$ for some $V_{i}^{\perp} \subset V^{\perp}$. By the product properties of tameness and holinks $\left((1)\right.$ in the corollary to 2.4) it is sufficient to show $\{0\} \subset\left(V^{\perp}-\bigcup V_{i}^{\perp}\right) \cup\{0\}$ has the desired properties. Radial contraction gives an almost strict deformation retraction, so $\{0\}$ is tame. Any map to a point is a fibration, so $\{0\}$ does have these properties, and the theorem is proved.

5.5. $h$-cobordisms. After some general remarks a sequence of lemmas are given, leading to a classification of $h$-cobordisms in Theorem 5.6.

An $h$-cobordism was defined above 1.8 to be a compact manifold homotopically stratified set $X$ with $\partial X=Y_{0} \cup Y_{1}$, such that if $A$ is a stratum of $X$ then the inclusions $Y_{i} \cap A \subset A$ are homotopy equivalences. The first remark is that the homotopy transversality in the definition of boundaries, and the global version of 3.5, imply that $X$ stratum-preserving deformation retracts to $Y_{i}$. Thus the non-stratified nature of the homotopy hypothesis is only apparent. We also remark that there is a version in which $Y_{i}$ are allowed to have boundary, and $\partial X=Y_{0} \cup Y_{1} \cup W$, where $W$ is an $h$-cobordism from $\partial Y_{0}$ to $\partial Y_{1}$. This is obtained from the statement given by 'absorbing $W$ into the filtration' in the manner described in 5.1 .

Lemma. A manifold homotopically stratified set is a layered set.

Proof. The hypotheses in Definition 4.5 are all included in the definition of manifold homotopically stratified set except finite dimensionality and reverse tameness of skeleta. Finite dimensionality follows from the finite dimensionality of the strata. Reverse tameness follows from Proposition 2.14 after absorbing the boundary into the filtration, as in 5.1 .

This means the invariant $\tau$ is defined for stratified homotopy equivalences of manifold homotopically stratified sets. According to the remark above, the inclusion $Y_{0} \subset X$ is a stratified homotopy equivalence. Not only does this mean $\tau$ is defined, but it induces an isomorphism of the homology groups in which such invariants lie. Therefore if $\left\{A_{i}\right\}$ denote stratum components of $Y_{0}$ we can consider $\tau\left(X, Y_{0}\right)$ as an element of $\sum_{i} H_{1}\left(\operatorname{cone} \delta A_{i}, \delta A_{i} ; \mathscr{S}\left(q_{i}\right)\right)$. (Or, we could use the negative of the torsion of the retraction $\left.X \rightarrow Y_{0}\right)$.

Lemma. Suppose $\left(X, Y_{0}, Y_{1}\right)$ is an h-cobordism with $\tau\left(X, Y_{0}\right)=0$, and a product structure $Z \simeq\left(Z \cap Y_{i}\right) \times I$ is given on a pure subset $Z$ which contains strata of dimension 5 or less. Then the product structure extends to a homeomorphism $X \simeq Y_{i} \times I$.

Proof. We proceed by induction, supposing that $Z$ contains the $n$-skeleton and then enlarging it to contain the $(n+1)$-skeleton. For the induction step it is sufficient to extend the product structure over a single stratum component $A$ with $\delta A \subset Z$. Denote $A \cap Y_{i}$ by $A_{i}$.

The product structure defines a stratum-preserving deformation retraction of $\delta \bar{A}$ to $\delta \bar{A}_{i}$, which by 3.5 can be extended to a stratum-preserving deformation 
retraction of $\bar{A}$ to $\bar{A}_{i}$. Denote by $r: \bar{A} \rightarrow \operatorname{cone} \delta A_{0}$ the composition of the retraction and the usual projection $\bar{A}_{0} \rightarrow$ cone $\delta A_{0}$. Using $r$ as control map and measuring in cone $\delta A_{0}$, we see that the deformations have radius 0 on $\delta A$. The Control Lemma 4.4 therefore implies (after radial reparameterization of the cone) that the stratum $\left(A ; A_{0}, A_{1}\right)$ is a controlled $h$-cobordism over the open cone. Lemma 4.9 identifies the invariant $\tau$ with the controlled torsion $q_{0}\left(A, A_{0}\right)$. By hypothesis this is trivial, so the controlled $h$-cobordism theorem [13 II, 1.6] implies there is a controlled product structure on $A$.

This product structure and the given one on $\delta A$ give a bijection $\bar{A} \rightarrow \bar{A}_{i} \times$ $I$. The control on the structure on $A$ implies that the projection to $\bar{A}_{i}$ is continuous, but the projection to $I$ may not be continuous initially. However it is straightforward to reparameterize the $I$ coordinate in the product structure on $A$ to make this projection continuous. The result is an extension of the product structure, as required for the lemma.

We recall that an $h$-cobordism $\left(X ; Y_{0}, Y_{1}\right)$ is said to be invertible if there is an $h$-cobordism $\left(W ; Y_{1}, Y_{2}\right)$ so that the "composition" $\left(X \cup_{Y_{1}} W ; Y_{0}, Y_{2}\right)$ has a product structure, and similarly there is an inverse for composition on the other side.

Lemma. If the 4-skeleton of an h-cobordism is invertible, the h-cobordism itself is invertible.

Proof. First we observe that invertibility is equivalent to the existence of stratified homeomorphisms $X-Y_{i} \simeq Y_{j} \times[0,1)$, where $\{i, j\}=\{0,1\}$. Recall that inverses are unique; if $X W \simeq Y_{0} \times I$ and $V X \simeq Y_{1} \times I$ then $V \simeq V(X W) \simeq$ $(V X) W \simeq W$. Therefore we can unambiguously denote the inverse of $X$ by $-X$. The infinite composition $X(-X) X(-X) \cdots$ can be associated two ways to give $Y_{0} \times[0, \infty) \simeq X\left(Y_{1} \times[0, \infty)\right)$. But since boundaries are collared the second space is $X-Y_{1}$. For the converse note that (again using the collaring) a homeomorphism $X-Y_{i} \simeq Y_{j} \times[0,1)$ gives a bicollared embedding of $X$ in $Y_{j} \times I$, whose complement is an inverse.

In applying the Boundary Collaring Theorem 1.2 in this argument we must assume boundaries of low dimensional strata are collared. However boundaries of invertible $h$-cobordisms are collared, so this is satisfied in the situation in the lemma.

Since the 4-skeleton is invertible, the above implies $X^{4} \simeq Y_{i}^{3} \times[0, \infty)$. The corollary in 5.2 applies to extend these to homeomorphisms $X \simeq Y_{i} \times[0, \infty)$, so $X$ is invertible.

5.6. Theorem. Suppose $Y_{0}$ is a compact manifold homotopically stratified set without boundary, $Z_{0} \subset Y_{0}$ is pure and contains strata of dimension 4 or less, and $\left(W ; Z_{0}, Z_{1}\right)$ is an invertible $h$-cobordism. Then $\tau$ defines an injection, from homeomorphism classes rel $Y_{0} \cup W$ of h-cobordisms of $Y_{0}$ extending $W$, to the obstruction group. 
In fact, if $\left\{A_{i}\right\}$ denotes the stratum components of $Y_{0}$ not in $Z$, then $\tau$ gives a bijection from these classes of $h$-cobordisms to

$$
\sum_{i} H_{1}\left(\operatorname{cone} \delta A_{i}, \delta A_{i} ; \mathscr{S}\left(q_{i}\right)\right) \text {. }
$$

Since the $h$-cobordism $W$ is fixed the other components of $\tau$ do not change and are irrelevant. The main point of the bijection is therefore the assertion that all invariants can be realized, as for layered sets in 1.10(2). The main step in this is a manifold version of the extension result in the corollary to 4.7. A treatment is planned in part V of [13]. Special cases of the realization can be done independently, for example when there is an extra $I$ factor, as in [18, Theorem 5].

Proof. Suppose $X, X^{*}$ are $h$-cobordisms of $Y_{0}$, both extending $W$ and with the same $\tau$ invariants. Let $-X$ be the inverse given by the invertibility lemma. Then $X^{*} \simeq X(-X) X^{*}$. But by additivity $\tau\left(-X X^{*}, Y_{1}\right)=\tau\left(-X, Y_{1}\right)+$ $\tau\left(X^{*}, Y_{0}\right)=-\tau\left(X, Y_{0}\right)+\tau\left(X, Y_{0}\right)=0$, so the product lemma implies $-X X^{*} \simeq$ $Y_{1} \times I$, and so $X^{*} \simeq X$. On the sub-h-cobordism $W$ this homeomorphism is the "uniqueness of inverses" homeomorphism $W \simeq(W(-W)) W \simeq W((-W) W) \simeq$ $W$, and so is isotopic to the identity. The isotopy extension theorem implies this isotopy can be extended to $X$. Composing with the inverse gives a homeomorphism $X^{*} \simeq X$ which is the identity on $Y_{0} \cup W$. This shows $h$-cobordisms with the same invariants are equivalent in the appropriate sense and completes the proof.

\section{REFERENCES}

1. E. Akin, Manifold phenomena in the theory of polyhedra, Trans. Amer. Math. Soc. 143 (1969), 413-473.

2. D. R. Anderson and W-C. Hsiang, The functors $K_{i}$ and pseudo-isotopies of polyhedra, Ann. of Math. 105 (1977), 201-223.

3. __ Extending combinatorial piecewise linearl structures on stratified spaces. II, Trans. Amer. Math. Soc. 260 (1980), 223-253.

4. W. Browder and F. Quinn, A surgery theory for G-manifolds and stratified sets, Manifolds, Tokyo 1973, Univ. Tokyo Press, Tokyo, 1975, pp. 27-36.

5. D. Carter, Lower K-theory of finite groups, Comm. Algebra 8 (1980), 1927-1937.

6. T. A. Chapman, Lectures on Hilbert cube manifolds, CBMS Regional Conf. Ser. in Math., no. 28, Amer. Math. Soc., Providence, R.I., 1976.

7. M. Cohen, A course in simple homotopy theory, Springer-Verlag, New York, 1973.

8. E. Fadell, Generalized normal bundles for locally-flat embeddings, Trans. Amer. Math. Soc. 114 (1965), 488-513.

9. F. T. Farrell and W-C. Hsiang, The Whitehead group of poly-( finite or cyclic) groups, J. London Math. Soc. 24 (1981), 308-324.

10. S. Ferry, A simple-homotopy approach to the finiteness obstruction, Lecture Notes in Math., no. 870, Springer, New York, 1981, pp. 73-81.

11. M. Freedman and F. Quinn, Topology of 4-manifolds, Princeton Univ. Press. (in preparation).

12. W-C. Hsiang and W. Pardon, When are topologically equivalent orthogonal transformations linearly equivalent?, Invent. Math. 68 (1982), 275-316. 
13. F. Quinn, Ends of maps, I, Ann. of Math. 110 (1979), 275-331; II, Invent. Math. 68 (1982), 353-424; III, J. Differential Geom. 17 (1982), 503-521; IV, Amer. J. Math. 108 (1986), 11391162.

14. __ Finite nilpotent group actions on finite complexes, Lecture Notes in Math., no. 657, Springer, New York, 1978, pp. 375-407.

15. __ Intrinsic skeleta and intersection homology of weakly stratified sets, Geometry and Topology, Lecture Notes in Math., vol. 105, Marcel Dekker, New York, 1987.

16. _ Algebraic K-theory of poly-( finite or cyclic) groups, Bull. Amer. Math. Soc. 12 (1985), 221-226.

17. L. Siebenmann, Deformation of homeomorphisms on stratified sets, Comment. Math. Helv. 47 (1972), 123-163.

18. M. Steinberger, The equivariant topological s-cobordism theorem, Invent. Math. 91 (1988), 61-104.

19. M. Steinberger and J. West, Equivariant h-cobordisms and finiteness obstructions, Bull. Amer. Math. Soc. 12 (1985), 217-220.

20. _ Controlled finiteness is the obstruction to equivariant handle decomposition (preprint).

21. R. Thom, Ensembles et morphismes stratifies, Bull. Amer. Math. Soc. 75 (1969), 240-284.

22. A. Verona, Stratified mappings-Structure and triangulability, Lecture Notes in Math., no. 1102, Springer, New York, 1984.

23. C. T. C. Wall, Finiteness conditions for CW complexes, I, Ann. of Math. 81 (1965), 56-69; II, Proc. Royal Soc. A 295 (1966), 129-139.

Department of Mathematics, Virginia Polytechnic Institute and State University, BLACKSBURG, VA 24061 